\title{
Su alcune proprietà delle topologie sequenziali e delle integrande normali $\left(^{*}\right)(* *)$.
}

\author{
ADA BOtTaRo ARUFFo
}

\begin{abstract}
Summary. - In this work we study some properties of the topology st, that is the strongest topology in which a sequence converges to $x$ if and only if it converges to $x$ in $\tau$. Such topology can be utilized in the study of the functions that are only sequentially continuous or sequentially lower semicontinuous with respect to $\tau$. We also study the behaviour of st with respect to some operations on the topologies. Moreover we prove theorems that are used in [3] to give some extensions of Scorza Dragoni's theorem.
\end{abstract}

\section{Introduzione.}

Molti autori ([1], [2], [5], [6], [8], [10], [13], [15], [16]) in lavori sul Calcolo delle Variazioni studiano la semicontinuità inferiore sequenziale delle funzioni in luogo della semicontinuità inferiore: questo uso è motivato dal fatto che è ovviamente in generale più semplice verificare sugli esempi tale proprietà riferita solo alle successioni. Spesso però in tali studi non vengono usati metodi strettamente topologici, in quanto la semicontinuità inferiore sequenziale non è vista come una proprietà rispetto ad una topologia. In special modo, quando si studiano problemi di Calcolo delle Variazioni relativi ad integrande definite su spazi di Banach, è utile poter fare sull'integranda ipotesi di semicontinuità inferiore sequenziale rispetto alla topologia debole in luogo di ipotesi di semicontinuità inferiore rispetto alla stessa topologia (tali concetti non sono invece ovviamente distinti nel caso della topologia forte, per la numerabilità delle basi di intorni). Nel presente lavoro, pertanto, uno degli scopi (nel §1) è quello di far vedere che una funzione è semicontinua inferiormente sequenzialmente su uno spazio topologico $(X, \tau)$ se e solo se

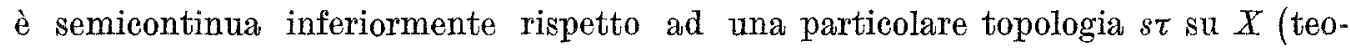
rema $1.1 \mathrm{~g})$ ) e di studiare proprietà di questa topologia. In particolare si studia il comportamento di tale topologia rispetto a varie operazioni sulle topologie, quali quelle di restrizione ad un sottospazio, di topologia finale e di prodotto (teoremi 1.1 d) $, 1.14,1.18,1.19,1.21,1.23)$. Inoltre, sempre nel $\S 1$, si danno vari esempi per mostrare che le ipotesi dei risultati forniti non sono sopprimibili. Nel $\S 2$

(*) Entrata in Redazione il 18 ottobre 1982 .

(**) Lavoro eseguito nell'ambito dell'Istituto per la Matematica Applicata del C.N.R., Genora. 
si provano invece (teoremi $2.1,2.3,2.4$ ) dei risultati che permetteranno in un prossimo lavoro ([3]) di fornire, una volta definita una proprietà detta "di Scorza Dragoni", delle estensioni del teorema di Scorza Dragoni (cfr. [10], Cap. VIII, Teorema 1.1 e Teorema del § 1.3) sulle integrande normali e sulle funzioni di Carathéodory. Inoltre nell'osservazione 2.5 si vede come sfruttare i risultati del $§ 1$ per ottenere altre condizioni sufficienti per avere le tesi dei teoremi $2.1,2.3,2.4$.

Desidero ringraziare il prof. J. P. CECCONI, che ha discusso con me i risultati del presente lavoro.

\section{0. - Notazioni.}

0.0 Notazioni. - Sia $Z$ uno spazio di Banach. Allora $|z|_{Z}$ indica la norma in $Z$ di $z \in Z, w(Z)$ la topologia debole su $Z$ indotta dalla dualità con il duale continuo di Z. Si abbrevierà O.N.C. per indicare "ortonormale completo", s.c.i. per indicare "semicontinua inferiormente", s.s.c.i. per indicare "sequenzialmente semicontinua inferiormente", inoltre [] indica la funzione "parte intera». Se $E, F, G, H$ sono insiemi allora $i_{E}: E \rightarrow E$ indica l'applicazione identica su $E, 2^{E}$ indica l'insieme delle parti di $E$; se $f: E \rightarrow F, g: G \rightarrow H$ sono applicazioni, allora $f \times g: E \times G \rightarrow F \times H$ indica l'applicazione tale che $(f \times g)(x, y)=(f(x), g(y))$ per ogni $(x, y) \in E \times G$, se inoltre $E_{0} \subset E, F_{0} \subset F$ e $F_{0} \supset f\left(E_{0}\right)$ allora $f /_{E_{0}}$ indica la restrizione di $f$ a $E_{0}$ e $f_{E_{0}, F_{0}}: E_{0} \rightarrow F_{0}$ indica l'applicazione tale che $f_{E_{0}, F_{0}}(x)=f(x)$ per ogni $x \in E_{0}$; se $D \subset$ $\subset E \times F$ allora $D_{x}=\{y \in F:(x, y) \in D\}$ per ogni $x \in E, D^{y}=\{x \in E:(x, y) \in D\}$ per ogni $y \in F, \operatorname{pr}_{E}: E \times F \rightarrow E$ è l'applicazione tale che $\operatorname{pr}_{E}(x, y)=x$ per ogni $(x, y) \in E \times F$ (per cui $\operatorname{pr}_{\mathbb{E}}(D)=\left\{x \in E: D_{x} \neq \emptyset\right\}$ ) e $\operatorname{pr}_{F}: E \times F \rightarrow F$ è definita in modo analogo. Se $(Z, \zeta),(W, \theta)$ sono spazi topologici, $E \subset Z$, allora $\left.\zeta\right|_{E}$ indica la topologia indotta su $E$ da $\zeta, \vec{E}^{\zeta}$ indica la chiusura di $E$ rispetto a $\zeta, \zeta \times \theta$ indica la topologia prodotto di $\zeta$ e di $\theta$ su $Z \times W, \mathscr{B}(\zeta)$ indiea la $\sigma$-algebra di Borel su $(Z, \zeta) ; \eta$ indica la topologia euclidea su $\boldsymbol{R}$ e $\delta$ la topologia discreta su $\boldsymbol{R}$. Se $(Z, d)$ è uno spazio pseudo-metrico, $a \in Z, r \in] 0, \infty\left[\right.$, allora $S_{Z}(a, r)=\{z \in Z: d(a, z)<r\}$. Se $[$ e Ath sono due $\sigma$-algebre rispettivamente su $T$ e su $S$ allora $\mathfrak{L} \times \mathcal{M}$ indica la più piccola $\sigma$-algebra su $T \times S$ che contiene la classe $\{A \times B: A \in \mathfrak{L}, B \in \mathcal{M}\}$, se $T_{0} \in \mathfrak{L}$ allora $\mathcal{L} / T_{0}$ indica la $\sigma$-algebra su $T_{0}$ che è restrizione della $\sigma$-algebra $\mathfrak{L}$ a $T_{0} ; \mathcal{L}(\boldsymbol{R})$ indica la $\sigma$-algebra di Lebesgue su $\boldsymbol{R}$; se $\mathfrak{A} \subset 2^{T}$ allora $\sigma(\mathcal{A})$ indica la $\sigma$-algebra su $T$ generata da $\mathcal{A}$ e $\tau(\mathcal{A})$ indica la topologia su $T$ generata da $\mathcal{A}$.

0.1 Definizione. - Siano $\mathcal{L} \sigma$-algebra su $T, \mu: \mathcal{L} \rightarrow[0, \infty]$ misura, $\mathcal{K} \subset \mathcal{L}$. Allora $\mu$ si dice $\mathcal{K}$-internamente regolare se per ogni $A \in \mathfrak{L}$ e per ogni $\varepsilon>0$ esiste $K_{\varepsilon} \in K$, $K_{\varepsilon} \subset A$ tale che $\mu\left(A \backslash K_{\varepsilon}\right)<\varepsilon$.

0.2 Definizione. - Uno spazio susliniano è uno spazio $S$ topologico $T_{2}$ tale che esistano uno spazio $P$ metrico completo e separabile e un'applicazione $\varphi$ continua e surgettiva, $\varphi: P \rightarrow \$$. 


\section{1. - Topologie sequenziali, topologie finali e $\sigma$-algebre.}

1.0 Definizioni. - Sia $(X, \varrho)$ spazio topologico.

a) Si indica con so la topologia sequenziale relativa a $\varrho$, che è così definita: $O$ è chiuso in $s \varrho$ se e solo se $C$ è sequenzialmente chiuso in $\varrho$ (e ciò̀ se e solo se per ogni $x_{n}, x \in X(n \in N)$, con $x_{n} \in C$ per ogni $n \in N, x_{n} \rightarrow x$ in $\varrho$ risulta che $\left.x \in C\right) ; 0$, equivalentemente, la topologia tale che: $A \in s Q$ se e solo se per ogni $x \in A$ e per ogni successione $\left(x_{n}\right)_{n \in \boldsymbol{N}}$ tale che $x_{n} \in X(n \in \boldsymbol{N}), x_{n} \rightarrow x$ in $\varrho$, esiste $n_{\boldsymbol{A}} \in \boldsymbol{N}$ per cui $x_{n} \in A$ se $n>n_{A}$ (cfr. $\left.[9], \S 7\right)$. Si dirà inoltre che $A$ è sequenzialmente aperto in $\varrho$ se $A \in s \varrho$.

b) Se $(Y, \sigma)$ è uno spazio topologico, $f:(X, \varrho) \rightarrow(Y, \sigma)$ si dice sequenzialmente continua [risp. sequenzialmente aperta] se $f:(X, s \varrho) \rightarrow(Y, s \sigma)$ è continua [risp. aperta], $g:(X, \varrho) \rightarrow[-\infty, \infty]$ si dice s.s.c.i. se $g:(X, s \varrho) \rightarrow[-\infty, \infty]$ è s.c.i.

1.1 Teorema. - Sia $(X, \varrho)$ spazio topologico e sia s $\varrho$ come nella definizione 1.0 a). Allora:

a) $s Q$ è la più fine tra tutte le topologie $\tau$ su $X$ tali che se $x_{n}, x \in X(n \in N)$, $x_{n} \rightarrow x$ in $\varrho$ allora $x_{n} \rightarrow x$ in $\tau$;

b) si ha $s \varrho \supset \varrho$ e se $\varrho$ è a base numerabile di intorni risulta che $s \varrho=\varrho$;

c) $s(s \varrho)=s \varrho$

d) se $E \subset X$ si ha che $\left.s\left(\left.\varrho\right|_{E}\right) \supset\left(s_{\varrho}\right)\right|_{E}$; inoltre vale anche l'altra inclusione se vale almeno una delle due seguenti condizioni:

$\left.d^{\prime}\right) E$ è chiuso in se,

$\left.d^{\prime \prime}\right) E \in s \varrho$.

Sia $(Y, \sigma)$ un altro spazio topologico. Allora:

e) $s(\varrho \times \sigma) \supset s \varrho \times s \sigma$;

f) se $f:(X, \varrho) \rightarrow(Y, \sigma)$, sono equivalenti i seguenti fatti:

$\left.f^{\prime}\right) f^{-1}(C)$ è sequenzialmente chiuso in $\varrho$ per ogni $O$ chiuso in $\sigma$,

$\left.f^{\prime \prime}\right)$ per ogni $x_{n}, x \in X(n \in N), x_{n} \rightarrow x$ in $\varrho$ si ha che $f\left(x_{n}\right) \rightarrow f(x)$ in $\sigma$,

$\left.f^{\prime \prime \prime}\right) f$ è sequenzialmente continua (ciò̀ $f^{-1}(O)$ è sequenzialmente chiuso in $\varrho$ per ogni $C$ sequenzialmente chiuso in $\sigma$ );

g) se $f:(X, \varrho) \rightarrow[-\infty, \infty]$, risulta che $f^{-1}([-\infty, a])$ è sequenzialmente chiuso in $\varrho$ per ogni $a \in[-\infty, \infty]$ (cioè $f$ è s.s.c.i.) se e solo se per ogni $x_{n}, x \in X$ $(n \in N), x_{n} \rightarrow x$ in $\varrho$, si ha che $f(x) \leqslant \liminf _{n \rightarrow \infty} f\left(x_{n}\right)$. 
Dimostrazione. - a) [9], $\S 8$.

b) Da $a)$ segue che $s \varrho \supset \varrho$ e dalla definizione di s@ segue la seconda parte della tesi.

e) $[9], \S 8$.

d) Si ha che $C$ è chiuso in $s\left(\varrho / /_{t}\right)$ se e solo se $C$ è sequenzialmente chiuso in $o / E$ e cioè se e solo se vale la seguente condizione:

(1.1.0) se $x_{n}, x \in E, x_{n} \in C$ per ogni $n \in N, x_{n} \rightarrow x$ in $\varrho$, risulta che $x \in C$,

mentre $C$ è chiuso in $(s Q) /_{E}$ se e solo se

(1.1.1) esiste $C^{\prime}$ sequenzialmente chiuso in $\varrho$ tale che $O=C^{\prime} \cap E$.

Ora è ovvio che (1.1.1) implica (1.1.0) e pertanto $s(\varrho / E) \supset(s \varrho) /_{E}$.

Valga ora $\left.d^{\prime}\right)$ e sia $C$ chiuso in $s(\varrho / E)$. Allora se $x_{n}, x \in X, x_{n} \in C$ per ogni $n \in N$, $x_{n} \rightarrow x$ in $\varrho$, si ha che $x_{n} \in E$ per ogni $n \in N$ e, visto che $E$ è chiuso in $s \varrho$, risulta che $x \in E$; pertanto, poichè $C$ verifica (1.1.0) si ottiene che $x \in C$ e quindi $C$ è sequenzialmente chiuso in $\varrho$ e cioè è chiuso in $s \varrho$ e quindi anche in $(s \varrho) /_{E}$.

Valga ora $\left.d^{\prime \prime}\right)$ e sia $A \in s(\varrho / E)$. Allora se $x_{n}, x \in X, x_{n} \in X \backslash A$ per ogni $n \in N$, $x_{n} \rightarrow x$ in $\varrho$, si ha che vale almeno uno dei due seguenti fatti:

i) esiste $\left(n_{k}\right)_{k \in N}$ successione strettamente crescente di naturali tale che $x_{n_{k}} \in E$ per ogni $k \in N$;

ii) esiste $\left(n_{k}\right)_{k \in N}$ successione strettamente crescente di naturali tale che $x_{n_{k}} \in$ $\in X \backslash E$ per ogni $k \in N$.

Se vale i) e se $x \in X \backslash E$ allora $x \in X \backslash A$; se invece $x \in E$ allora, visto che $E \backslash A$ verifica (1.1.0), risulta che $x \in X \backslash A$. Se vale ii) allora $x \in X \backslash E$, poichè $X \backslash E$ è sequenzialmente chiuso in $\varrho$, e quindi $x \in X \backslash A$. Pertanto in ogni caso si è trovato che $x \in X \backslash A$; ne segue che $A \in s \varrho$ e quindi $\left.A \in(s \varrho)\right|_{E}$.

e) Se $C$ è chiuso in $s \varrho$ si ha che $C$ è sequenzialmente chiuso in $\varrho$ e quindi $C \times Y$ è sequenzialmente chiuso in $\varrho \times \sigma$ e cioè chiuso in $s(\varrho \times \sigma)$, analogamente se $D$ è chiuso in $s \sigma$ risulta che $X \times D$ è chiuso in $s(\varrho \times \sigma)$; utilizzando ora il fatto che ogni chiuso di $s \varrho \times s \sigma$ si può scrivere come intersezione di unioni finite di insiemi appartenenti alla classe $\{C \times Y: C$ chiuso in $s \varrho\} \cup\{X \times D: D$ chiuso in $s \sigma\}$, si deduce che $s \varrho \times$ $\times s \sigma \subset s(\varrho \times \sigma)$.

f) Valga $\left.f^{\prime}\right)$. Allora, se $x_{n}, x \in X(n \in N), x_{n} \rightarrow x$ in $\varrho$ e se $A \in \sigma$ ed è tale che $f(x) \in A$, risulta che $f^{-1}(X \backslash A)$ è sequenzialmente chiuso in $\varrho$ e $x \notin f^{-1}(Y \backslash A)$; pertanto, visto che $x_{n} \rightarrow x$ in $\varrho$, esiste $n_{A} \in N$ tale che se $n>n_{d}$ sia $x_{n} \notin f^{-1}(Y \backslash A)$; per cui $f\left(x_{n}\right) \in A$ per ogni $n>n_{A}$ e quindi $f\left(x_{n}\right) \rightarrow f(x)$ in $\sigma$. Pertanto vale $\left.f^{\prime \prime}\right)$. Valga ora $\left.f^{\prime \prime}\right)$. Sia $C$ sequenzialmente chiuso in $\sigma$ e siano $x_{n} \in f^{-1}(C)(n \in \mathbf{N}), x \in X, x_{n} \rightarrow x$ 
in $\varrho$. Allora $f\left(x_{n}\right) \rightarrow f(x)$ in $\sigma$ e $f\left(x_{n}\right) \in C$ per ogni $n \in N$; pertanto $f(x) \in C$ e quindi $x \in f^{-1}(C)$, per cui vale $\left.f^{\prime \prime \prime}\right)$. Ora si conclude osservando che da $b$ ) segue che $\left.f^{\prime \prime \prime}\right)$ implica $\left.f^{\prime}\right)$.

L'equivalenza tra $f^{\prime}$ ) ed $f^{\prime \prime}$ ) è provata anche in [18], (1) e quella tra $f^{\prime \prime}$ ) ed $f^{\prime \prime \prime}$ ) $\zeta$ provata anche in [19], Teorema 3.1.

g) $\operatorname{Sia} f^{-1}([-\infty, a])$ sequenzialmente chiuso in $\varrho$ per ogni $a \in[-\infty, \infty]$. Allora, se $x_{n}, x \in X(n \in N), x_{n} \rightarrow x$ in $\varrho$ e se $b \in[-\infty, \infty], b>\liminf _{n \rightarrow \infty} f\left(x_{n}\right)$, poichè $f^{-1}([-\infty, b])$ è sequenzialmente chiuso in $\varrho$, risulta che $f(x) \leqslant b$, per eui $f(x) \leqslant \liminf _{n \rightarrow \infty} f\left(x_{n}\right)$. Viceversa per ogni $x_{n}, x \in X(n \in N), x_{n} \rightarrow x$ in $\varrho$, si abbia che $f(x) \leqslant \liminf _{n \rightarrow \infty} f\left(x_{n}\right)$. Sia $a \in[-\infty, \infty]$ e siano $x_{n} \in f^{-1}([-\infty, a])(n \in N), x \in X, x_{n} \rightarrow x$ in $\varrho$. Allora $f(x) \leqslant$ $\leqslant \liminf _{n \rightarrow \infty} f\left(x_{n}\right) \leqslant a$ e quindi $x \in f^{-1}([-\infty, a])$.

1.2 EsEMPI. - a) Si noti che, se $\varrho$ è una topologia su $X$, se come nella definizione $1.0 a)$, può accadere che sia $8 \varrho \supsetneqq \varrho$.

Basta ad esempio considerare $X$ spazio di Hilbert separabile e di dimensione infinita su $\boldsymbol{R}, \varrho=w(X)$. Siano $\left\{e_{n}: n \in \boldsymbol{N}\right\}$ un sistema O.N.C. in $X$ e $O=\left\{\sqrt{n+1} e_{n}: n \in \boldsymbol{N}\right\}$. Allora $C$ è chiuso in $s w(X)$ perchè è sequenzialmente chiuso in $w(X)$ (infatti ogni successione convergente in $w(X)$ è limitata e $w(X)$ è $T_{2}$, per cui ogni successione di elementi di $C$ e convergente è definitivamente costante), ma $C$ non è chiuso in $w(X)$ poichè $0 \in \overline{\{\sqrt{n+1}}_{\left.e_{n}: n \in N\right\}}{ }^{v(X)}$ (efr. [12], Soluzione del problema 21).

(Un altro esempio di topologia $\varrho$ tale che $s_{\varrho} \supsetneqq \varrho$ è dato in [9], § 12).

b) Si noti che, se $\varrho$ è una topologia su $X$ e se $B \subset X$, non è detto che sia

$$
\bar{B}^{s e}=\left\{x \in X \text { : esistono } x_{n} \in B(n \in \mathbb{N}) \text { per cui } \lim _{n \rightarrow \infty} x_{n}=x \text { in } \varrho\right\} .
$$

In particolare, quindi, non è nemmeno detto che se sia a base numerabile di intorni. (Cfr., per osservazioni sull'argomento, [9], § 15).

Basta considerare $X,\left\{e_{n}: n \in N\right\}, \varrho$ come in $\left.a\right), B=\left\{n e_{l k}+e_{n}: n, k \in N\right\}$. Allora, tenendo conto di a) del teorema 1.1, per ogni $n \in N$ si ha che $\lim _{k \rightarrow \infty}\left(n e_{k}+e_{n}\right)=e_{n}$ in $s w(X)$, da cui $e_{n} \in \bar{B}^{s w(X)}$; inoltre $\lim _{n \rightarrow \infty} e_{n}=0$ in $s w(X)$ e pertanto $0 \in \bar{B}^{s w(X)}$. D'altra parte $0 \notin\left\{x \in X\right.$ : esistono $x_{n} \in B(n \in N)$ per cui $\lim _{n \rightarrow \infty} x_{n}=x$ in $\left.w(X)\right\}$. Se infatti per assurdo esistessero $n_{i}, k_{i} \in N(i \in N)$ tali che $\lim _{i \rightarrow \infty}\left(n_{i} e_{k_{i}}+e_{n_{i}}\right)=0$ in $w(X)$, esisterebbe $H>0$ per cui $\left|n_{i} e_{k_{i}}+e_{n_{i}}\right|_{X} \leqslant H$ per ogni $i \in N$ e pertanto $n_{i}=\mid n_{i} e_{k_{i} \mid X} \leqslant$ $\leqslant\left|n_{i} e_{k_{i}}+e_{n_{i}}\right| x+\left|e_{n_{i}}\right|_{x} \leqslant H+1$ per ogni $i \in N$; dunque $\left\{n_{i}: i \in N\right\}$ sarebbe un insieme finito e quindi esisterebbero $n \in N$ e $\left(h_{i}\right)_{i \in N}$ estratta da $\left(k_{i}\right)_{i \in N}$ tali che $\left(n e_{h_{t}}+e_{n}\right)_{i \in N}$ sia sottosuccessione di $\left(n_{i} e_{k t}+e_{n_{i}}\right)_{i \in N}$; ora, se esistesse $K>0$ tale che $h_{i} \leqslant K$ per ogni $i \in N$, allora esisterebbe $h \in N$ tale che $\left(n e_{h}+e_{n}\right)_{i \in N}$ sia estratta da $\left(n_{i} e_{k_{t}}+e_{n_{i}}\right)_{i \in N}$ e poichè $n e_{h}+e_{n} \neq 0$ ciò è assurdo; se invece $\left(h_{i}\right)_{i \in N}$ non fosse limitata, allora esisterebbe un'estratta $\left(l_{i}\right)_{i \in N}$ di $\left(h_{i}\right)_{i \in N}$ tale che $l_{i} \rightarrow \infty$ e pertanto $\lim _{i \rightarrow \infty}\left(n e_{l_{i}}+e_{n}\right)=e_{n}$ in $w(X)$ e ciò è assurdo. (L'insieme qui considerato era già stato 
usato in $[17](\S I, 1)$ per dare un esempio di insieme $I$ tale che non esistano successioni ad elementi in $I$ convergenti a 0 in $w(X)$ e tale che 0 sia punto di accumulazione di $I$ in $w(X)$ (la dimostrazione di quest'ultimo fatto data sopra è un po' meno laboriosa di quella fornita in [17])).

c) Si noti che, nelle condizioni di $d$ ) del teorema 1.1 , se $E$ non è nè aperto nè chiuso in $s \varrho$ si può avere $s\left(\varrho / /_{E}\right) \neq(s \varrho) / E$.

Basta considerare $X,\left\{e_{n}: n \in \boldsymbol{N}\right\}, \varrho, B$ come in $\left.b\right), E=B \cup\{0\}$; allora dalle considerazioni fatte in $b$ ) segue che $0 \notin\left\{x \in E\right.$ : esistono $x_{n} \in B(n \in N)$ per eui $\lim _{n \rightarrow \infty} x_{n}=x$ in $\varrho\}$; pertanto $B$ verifica la condizione (1.1.0), ma non può verificare la condizione (1.1.1) visto che se $B^{t}$ è sequenzialmente chiuso in $\varrho, B^{\prime} \supset B$, si ha che $B^{\prime} \supset \bar{B}^{\text {se }}$ e quindi per $b)$ risulta che $0 \in B^{\prime}$. Allora $B$ è chiuso in $s(\varrho / E)$, ma non è chiuso in $(s \varrho) /{ }_{B}$.

(Un altro esempio in cui si ha che $s(\varrho / E) \neq(s \varrho) /_{E}$ è dato in [20] (esempio 1.8)).

1.3 Esempr. - a) Sia $H$ spazio di Hilbert separabile e di dimensione infinita su $\boldsymbol{R}, \infty \notin H$ e $\tau$ la topologia su $H \cup\{\infty\}$ generata da $w(H) \cup\left\{\{\infty\} \cup\left(H \backslash \overline{S_{H}(0, r)}\right)\right.$ : $r>0\}$ (cioè $(H \cup\{\infty\}, \tau)$ la compattificazione con un punto di $(H, w(H)))$. Allora:

$\left.a^{\prime}\right)$ la convergenza delle successioni in $\tau$ ha unicità del limite;

$\left.a^{\prime \prime}\right) s \tau$ non è $T_{2}$.

(Un altro esempio di convergenza con unicità del limite e tale che la topologia associata non sia $T_{2}$ è dato in [9], § 12).

$\left.a^{\prime}\right)$ Siano $x_{n}, x, y \in H \cup\{\infty\}(n \in \boldsymbol{N}), x_{n} \rightarrow x$ e $x_{n} \rightarrow y$ in $\tau$. Allora, se esiste $\left(x_{n_{k}}\right)_{k \in N}$ estratta da $\left(x_{n}\right)_{n \in N}$ tale che $x_{n_{k}}, x \in H$ [risp. $\left.x_{n_{k}}, y \in H\right](k \in N)$, si ha che $x_{n_{k}} \rightarrow x$ [risp. $x_{n_{k}} \rightarrow y$ ] in $w(H)$ e quindi $\left(x_{n_{k}}\right)_{k \in N}$ è limitata in $H$; pertanto, visto che $x_{n_{k}} \rightarrow y$ [risp. $x_{n_{k}} \rightarrow x$ ] in $\tau$, anche $y \in H$ [risp. $x \in H$ ] e per l'unicità del limite in $w(H)$ si ha che $x=y$. Se invece non esiste $\left(x_{n_{k}}\right)_{k \in N}$ estratta da $\left(x_{n}\right)_{n \in N}$ nè tale che $x_{n_{k}}, x \in H$, nè tale che $x_{n_{k}}, y \in H(k \in N)$, allora vale almeno uno dei due seguenti fatti:

i) $x=y=\infty$;

ii) esiste $n_{0} \in N$ tale ehe $x_{n}=\infty$ per ogni $n>n_{0}$.

Nel caso i) si ha subito che $x=y$. Nel caso ii) si ha di nuovo che $x=y=\infty$, perchè se $x \in H$ [risp. $y \in H$ ] esiste $U \in w(H)$ tale che $x \in U$ [risp. $y \in U$ ], quindi $U \in \tau$ e pertanto $x_{n}$ non tende a $x$ in $\tau$ [risp. $x_{n}$ non tende a $y$ in $\tau$ ].

$\left.a^{\prime \prime}\right)$ Per [14] (Cap. 5, Teorema 21) $\tau$ non è $T_{2}$, poichè $(H, w(H))$ non è localmente compatto. D'altra parte, se $k \in \boldsymbol{R}$ e se $\left\{e_{n}: n \in N\right\}$ è un sistema O.N.C. in $H$, si ha che $\lim _{n \rightarrow \infty} k e_{n}=0$ in $w(H)$ e quindi anche in $\tau$; allora $\lim _{n \rightarrow \infty} k e_{n}=0$ in $s \tau$; pertanto 0 non può avere in $s \tau$ intorni che siano limitati in $H$ e quindi, tenendo conto del fatto che se $O$ è sequenzialmente chiuso in $\tau, \infty \notin C$, allora $O$ è limitato, si ha che non esistono $U_{1}, U_{2} \in s \tau$ tali che $U_{1} \cap U_{2}=\emptyset, 0 \in U_{1}, \infty \in U_{2}$. 
b) Si noti che, nelle condizioni di $e$ ) del teorema 1.1, può accadere che sia $s(\varrho \times \sigma) \neq s \varrho \times 8 \sigma$.

Basta considerare $X=Y=H \cup\{\infty\}, \varrho=\sigma=s \tau$, ove $H, \infty, \tau$ sono come in a). Allora $D=\{(x, x): x \in H \cup\{\infty\}\}$ è chiuso in $s(s \tau \times s \tau)$ ma non in $s \tau \times s \tau$ (e si noti che $s(s \tau) \times s(s \tau)=s \tau \times s \tau$ per $c$ ) del teorema 1.1). Infatti $D$ è sequenzialmente chiuso in $s \tau \times s \tau$ per $a^{\prime}$ ) e non è chiuso in $s \tau \times s \tau$ per $\left.a^{\prime \prime}\right)$.

(Un altro esempio in cui si ha che $s(\varrho \times \sigma) \neq s \varrho \times s \sigma$ (e con $\varrho=s \varrho . \sigma=s \sigma$,

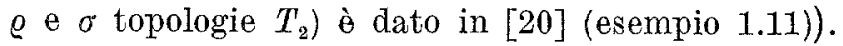

1.4 Lemma. - Siano $(X, \varrho),(Y, \sigma),(Z, \zeta),(W, \theta)$ spazi topologici, $\varphi:(X, \varrho) \rightarrow$ $\rightarrow(Z, \zeta)$ e $\psi:(Y, \sigma) \rightarrow(W, \theta)$ aperte. Allora $\varphi \times \psi:(X \times Y, \varrho \times \sigma) \rightarrow(Z \times W, \zeta \times \theta)$ ѐ aperta.

Dimostrazione. - Siano $A \in \varrho \times \sigma$ e $(z, w) \in(\varphi \times \psi)(A)$. Allora esiste $(x, y) \in A$ tale che $\varphi(x)=z, \psi(y)=w$. Per definizione di topologia prodotto esistono $B \in \varrho$, $C \in \sigma$ tali che $(x, y) \in B \times C \subset A$. Allora $(z, w)=(p(x), \psi(y)) \in \varphi(B) \times \psi(C) \in \zeta \times \theta$ e quindi $(\varphi \times \psi)(A) \supset(\varphi \times \psi)(B \times C)=\varphi(B) \times \psi(C) \grave{\mathrm{e}}$ un intorno di $(z, w)$.

1.5 TEorema. - $a$ ) Siano $(X, \varrho)$ e $(Y, \sigma)$ spazi topologici, di cui uno almeno sia a base numerabile di aperti. Allora $\mathfrak{B}(\varrho \times \sigma)=\mathfrak{B}(\varrho) \times \mathfrak{B}(\sigma)$.

b) Siano $X, Y$ insiemi, $\mathcal{A} \subset 2^{X}, \mathcal{C} \subset 2^{Y}$ tali che $X \in \mathcal{A}, Y \in \mathfrak{C}$, M la $\sigma$-algebra generata da $\{A \times C: A \in \mathcal{A}, C \in \mathcal{C}\}$. Allora $\sigma(\mathcal{A}) \times \sigma(\mathbb{C})=\mathcal{A}$.

c) Siano $X$ insieme, $\mathcal{A} \subset 2^{X}, \mathcal{H}=\cup(\sigma(\mathcal{C}): \mathcal{C} \subset \mathcal{A}, \mathcal{C}$ al più numerabile). Allora $\sigma(\mathcal{A})=\mathcal{M}$.

Dimostrazione. - a) L'inclusione $\mathfrak{B}(\varrho) \times \mathfrak{B}(\sigma) \subset \mathfrak{B}(\varrho \times \sigma)$ vale con la sola ipotesi che $(X, \tau)$ e $(Y, \sigma)$ siano spazi topologici. Per provarla basta dimostrare che, se $A \in \mathfrak{B}(\varrho)$ e $B \in \mathfrak{B}(\sigma)$, allora $A \times B \in \mathfrak{B}(\varrho \times \sigma)$ e, poichè $A \times B=(A \times Y) \cap(X \times B)$, basta verificare che $A \times Y, X \times B \in \mathscr{B}(\varrho \times \sigma)$. Ma le due applicazioni $\mathrm{pr}_{X}: X \times Y \rightarrow X$, $\mathrm{pr}_{Y}: X \times Y \rightarrow Y$ sono continue e quindi tali che $\operatorname{pr}_{X}^{-1}(A)=A \times Y \in \mathfrak{B}(\varrho \times \sigma)$ e $\operatorname{pr}_{T}^{-1}(B)=X \times B \in \Re(\varrho \times \sigma)$.

Si supponga ora che per esempio $(Y, \sigma)$ sia a base numerabile di aperti. Per provare che $\mathfrak{B}(\varrho \times \sigma) \subset \mathfrak{B}(\varrho) \times \mathfrak{B}(\sigma)$, basta dimostrare che se $U \in \varrho \times \sigma$ allora $U \in \mathfrak{B}(\varrho) \times$ $\times \Re(\sigma)$. Ma per definizione di topologia prodotto esistono $A_{i} \in \varrho, B_{i} \in \sigma(i \in I)$ tali che sia $U=\bigcup_{i \in I}\left(A_{i} \times B_{i}\right)$. Inoltre per l'ipotesi fatta $\mathrm{su}(Y, \sigma)$ esiste $\left\{O_{n}: n \in N\right\}$ base numerabile di aperti per $(Y, \sigma)$ e quindi, se per ogni $i \in I$ si definisce $\mathbf{N}_{i}=\{n \in N$ : $\left.C_{n} \subset B_{i}\right\}$, si ha:

$$
U=\bigcup_{i \in I}\left(A_{i} \times\left(\bigcup_{n \in N_{i}} C_{n}\right)\right)=\bigcup_{n \in N}\left(\left(\bigcup_{i \in I_{n}} A_{i}\right) \times C_{n}\right)
$$

ove si ̀̀ posto $I_{n}=\left\{i \in I: n \in N_{i}\right\}$ per ogni $n \in N$; ora per ogni $n \in N$ si ha che $\bigcup_{i \in I_{n}} A_{i} \in \varrho$ e $O_{n} \in \sigma$ per cui $\left(\bigcup_{i \in I_{n}} A_{i}\right) \times C_{n} \in \mathfrak{B}(\varrho) \times \mathfrak{B}(\sigma)$ e pertanto $U \in \mathfrak{B}(\varrho) \times \mathfrak{B}(\sigma)$. 
b) Poichè $\sigma(\mathcal{A}) \times \sigma(\mathcal{C})$ è una $\sigma$-algebra che contiene $\{A \times C: A \in \mathcal{A}, C \in \mathfrak{C}\}$, si ha che $\sigma(\mathfrak{A}) \times \sigma(\mathcal{C}) \supset \mathcal{A}$. Per concludere basta ora provare che, se $A \in \sigma(\mathcal{A})$ e $C \in \sigma(\mathcal{C})$, allora $A \times Y, X \times O \in \mathcal{H}$, poichè da ciò segue che $A \times O=(A \times Y) \cap(X \times O) \in \mathcal{N}$, da cui $\sigma(\mathcal{A}) \times \sigma(\mathcal{C}) \subset \mathcal{M}$. Ora $\{A: A \times Y \in \mathcal{M}\} \supset \mathcal{A}$ ed è una $\sigma$-algebra, per cui $A \times Y \in \mathscr{M}$ per ogni $A \in \sigma(\mathcal{A})$. Analogamente $X \times C \in \mathcal{M}$ per ogni $C \in \sigma(\mathcal{C})$.

c) Si ha che $\mathcal{H}$ è una $\sigma$-algebra (se $A \in \sigma(\mathcal{C})$ allora $X \backslash A \in \sigma(\mathcal{C})$ e se $A_{n} \in \sigma\left(\mathcal{C}_{n}\right)$ $(n \in N)$ allora $A_{m} \in \sigma\left(\bigcup_{n \in N} \mathcal{C}_{n}\right)$ per ogni $\left.m \in N\right)$. Si ha inoltre che $\mathcal{A} \subset \mathcal{A}$ visto che se $A \in \mathcal{A}$ allora $A \in \sigma(\{A\})$; quindi $\sigma(\mathcal{A}) \subset \mathcal{A}$. D'altra parte se $A \in \mathcal{M}$ allora esiste C C $\mathcal{A}$ tale che $A \in \sigma(\mathcal{C}) \subset \sigma(\mathcal{A})$, per cui $\mathscr{H} \subset \sigma(\mathfrak{A})$ e si conclude.

1.6 Esempi. - a) La tesi di a) del teorema 1.5 puo non essere vera se non si fa l'ipotesi che almeno uno tra gli spazi $(X, \varrho)$ e $(Y, \sigma)$ sia a base numerabile di aperti.

Basta considerare un insieme $X$ che abbia cardinalità maggiore di $2^{N_{0}}$ e $\varrho$ la topologia discreta su $X$. Allora si ha che $\varrho \times \varrho$ è la topologia discreta su $X \times X$ e pertanto $\Delta=\{(x, x): x \in X\} \in \mathfrak{B}(\varrho \times \varrho)$. D'altra parte $\Delta \notin \mathcal{B}(\varrho) \times \mathfrak{B}(\varrho)$. Se infatti per assurdo $\Delta \in \mathscr{B}(\varrho) \times \mathfrak{B}(\varrho)$, allora, poichè per $b)$ del teorema 1.5 risulta che $\mathfrak{B}(\varrho) \times \mathfrak{B}(\varrho)=$ $=\sigma(\{A \times B: A, B \in \varrho\})$, per $c)$ del teorema 1.5 esiste $\mathcal{C} \subset\{A \times B: A, B \in \varrho\}, \mathcal{C}$ al più numerabile tale che $\Delta \in \sigma(\mathcal{C})$ e quindi, se $\mathscr{D}=\{D \in \varrho$ : esiste $E \in \varrho$ per cui almeno uno tra $D \times E$ ed $E \times D$ è in $C\}$, si ha che $D$ è al più numerabile e $\Delta \in \sigma(\{A \times B$ : $A, B \in \mathbb{D}\})$. Dunque l'applicazione $\varphi: x \in X \mapsto\{D \in \mathbb{D}: x \in D\} \in 2^{\mathbb{D}}$ è iniettiva, poichè se $x_{1}, x_{2} \in X$ sono tali che $\varphi\left(x_{1}\right)=\varphi\left(x_{2}\right)$ allora $\sigma(\{A \times B: A, B \in \mathbb{D}\}) \subset\{E \subset X \times X$ : $\left(x_{1}, x_{1}\right) \in E$ se e solo se $\left.\left(x_{1}, x_{2}\right) \in E\right\}$ in quanto l'insieme a secondo membro è una $\sigma$-algebra che contiene $\{A \times B: A, B \in \mathcal{D}\}$, quindi $\Delta \in\left\{E \subset X \times X:\left(x_{1}, x_{1}\right) \in E\right.$ se e solo se $\left.\left(x_{1}, x_{2}\right) \in E\right\}$, da cui $x_{1}=x_{2}$. Ma se $\varphi$ è iniettiva, allora la cardinalità di $X$ è minore o uguale di quella di $2^{D}$ il che è assurdo.

b) La tesi di $b$ ) del teorema 1.5 può non essere vera se non si fa l'ipotesi che $X \in \mathfrak{t}, Y \in \mathcal{C}$.

Basta considerare $X=Y=\{0,1\}, \mathcal{A}=\{\{0\},\{0,1\}\}, \mathcal{C}=\{\{0\}\}$. Risulta allora: $\sigma(\mathfrak{A})=2^{X}, \sigma(\mathcal{C})=2^{Y}, \sigma(\mathcal{A}) \times \sigma(\mathcal{C})=2^{X \times Y}, \operatorname{ma} \mathcal{M}=\{\emptyset,\{(0,0)\},\{(0,0),(1,0)\},\{(1$, $0)\},\{(0,1),(1,1)\},\{(0,0),(0,1),(1,1)\},\{(1,0),(0,1),(1,1)\}, X \times Y\}$ e pertanto $\{(0,1)\}$ e $\{(1,1)\}$ non sono in $\mathcal{H}$.

1.7 Lemma. - Siano $Z, W, Z_{i}, W_{i}$ insiemi, $\mathcal{A} \subset 2^{Z}, \mathcal{C} \subset 2^{W}, \mathfrak{A}_{i} \subset 2^{Z i}, \mathfrak{C}_{i} \subset 2^{W_{i}}, p: Z \rightarrow$ $\rightarrow W, p_{i}: Z_{i} \rightarrow W_{i}, \hat{p}: 2^{Z} \rightarrow 2^{W}, \hat{p}_{i}: 2^{Z_{i}} \rightarrow 2^{W_{i}}, \widehat{p_{1} \times p_{2}}: 2^{Z_{1} \times Z_{2}} \rightarrow 2^{W_{1}} \times W_{2}$ tali che $\hat{p}(A)=$ $=p(A)$ per ogni $A \in 2^{Z}, \hat{p}_{i}(E)=p_{i}(E)$ per ogni $E \in 2^{Z_{i}}, \widehat{p_{1} \times p_{2}}(D)=\left(p_{1} \times p_{2}\right)(D)$ per ogni $D \in 2^{Z_{1} \times Z_{2}}(i=1,2)$. Allora:

a) $p^{-1}(\sigma(\mathcal{C})) \subset \sigma\left(p^{-1}(\mathcal{C})\right)$;

b) $p^{-1}(\tau(\mathfrak{C})) \subset \tau\left(p^{-1}(\mathcal{C})\right)$; 
c) se $p^{-1}(p(A))=A$ per ogni $A \in \mathcal{A}$, si ha che $p^{-1}(\sigma(\hat{p}(\mathcal{A}))) \subset \sigma(\mathcal{A}), p^{-1}(p(A))=A$ per ogni $A \in \sigma(\mathcal{A})$ e, se $Z \in \mathcal{A}$, si ha anche che $\hat{p}(\sigma(\mathcal{A})) \subset \sigma(\hat{p}(\mathfrak{A}))$;

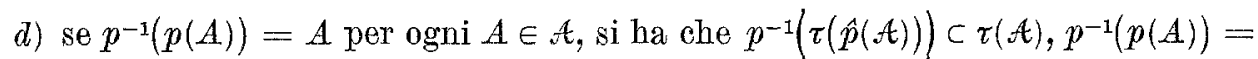
$=A$ per ogni $A \in \tau(\mathcal{A})$ e, se $Z \in \mathcal{A}$, si ha anche che $\hat{p}(\tau(\mathcal{A})) \subset \tau(\hat{p}(\mathfrak{A}))$;

e) se $p_{i}^{-1}\left(p_{i}(A)\right)=A$ per ogni $A \in \mathcal{A}_{i}(i=1,2)$, si ha che $\left(p_{1} \times p_{2}\right)^{-1}\left(\left(p_{1} \times\right.\right.$ $\left.\left.\times p_{2}\right)(A)\right)=A$ per ogni $A \in\left\{A_{1} \times A_{2}: A_{i} \in \mathcal{A}_{i}(i=1,2)\right\}$;

f) se $p_{i}^{-1}\left(\mathcal{C}_{i}\right) \subset \mathcal{A}_{i}(i=1,2)$, si ha che $\left(p_{1} \times p_{2}\right)^{-1}\left(\left\{C_{1} \times C_{2}: C_{i} \in \mathcal{C}_{i}(i=1,2)\right\}\right) \subset$ $\subset\left\{A_{1} \times A_{2}: A_{i} \in \mathcal{A}_{i}(i=1,2)\right\}$;

g) se $\hat{p}_{i}\left(\mathcal{A}_{i}\right) \subset \mathcal{C}_{i}(i=1,2)$, si ha che $\widehat{p_{1} \times p_{2}}\left(\left\{A_{1} \times A_{2}: A_{i} \in \mathcal{A}_{i}(i=1,2)\right\}\right) \subset$ c $\left\{C_{1} \times C_{2}: O_{i} \in \mathcal{C}_{i}(i=1,2)\right\}$

h) si ha che $\left(p_{1} \times p_{2}\right)^{-1}\left(\sigma\left(\mathcal{C}_{1}\right) \times \sigma\left(\mathcal{C}_{2}\right)\right) \subset \sigma\left(p_{1}^{-1}\left(\mathcal{C}_{1}\right)\right) \times \sigma\left(p_{2}^{-1}\left(\mathcal{C}_{2}\right)\right)$;

i) si ha che $\left(p_{1} \times p_{2}\right)^{-1}\left(\tau\left(\mathcal{C}_{1}\right) \times \tau\left(\mathcal{C}_{2}\right)\right) \subset \tau\left(p_{1}^{-1}\left(\mathcal{C}_{1}\right)\right) \times \tau\left(p_{2}^{-1}\left(\mathfrak{C}_{2}\right)\right)$;

j) se $p_{i}^{-1}\left(p_{i}(A)\right)=A$ per ogni $A \in \mathcal{A}_{i}(i=1,2)$, si ha che $\left(p_{1} \times p_{2}\right)^{-1}\left(\sigma\left(\hat{p}_{1}\left(\mathcal{A}_{1}\right)\right) \times\right.$ $\left.\times \sigma\left(\hat{p}_{2}\left(\mathfrak{t}_{2}\right)\right)\right) \subset \sigma\left(\mathfrak{t}_{1}\right) \times \sigma\left(\mathfrak{A}_{2}\right),\left(p_{1} \times p_{2}\right)^{-1}\left(\left(p_{1} \times p_{2}\right)(A)\right)=A$ per ogni $A \in \sigma\left(\mathcal{A}_{1}\right) \times$ $\times \sigma\left(\mathfrak{A}_{2}\right)$ e se $Z_{i} \in \mathfrak{A}_{i} \quad(i=1,2)$ si ha anche che $\widehat{p_{1} \times p_{2}}\left(\sigma\left(\mathcal{A}_{1}\right) \times \sigma\left(\mathcal{A}_{2}\right)\right) c$ $\operatorname{C\sigma }\left(\hat{p}_{1}\left(\mathscr{A}_{1}\right)\right) \times \sigma\left(\hat{p}_{2}\left(\mathcal{A}_{2}\right)\right)$

k) se $p_{i}^{-1}\left(p_{i}(A)\right)=A$ per ogni $A \in \mathcal{A}_{i}(i=1,2)$, si ha che $\left(p_{1} \times p_{2}\right)^{-1}\left(\tau\left(\hat{p}_{1}\left(\mathcal{A}_{1}\right)\right) \times\right.$ $\left.\times \tau\left(\hat{p}_{2}\left(\mathcal{A}_{2}\right)\right)\right) \subset \tau\left(\mathfrak{H}_{1}\right) \times \tau\left(\mathcal{A}_{2}\right),\left(p_{1} \times p_{2}\right)^{-1}\left(\left(p_{1} \times p_{2}\right)(A)\right)=A$ per ogni $A \in \tau\left(\mathcal{A}_{1}\right) \times$ $\times \tau\left(\mathfrak{A}_{2}\right)$ e se $Z_{i} \in \mathfrak{A}_{i} \quad(i=1,2)$ si ha anche che $\widehat{p_{1} \times p_{2}}\left(\tau\left(\cdot \mathfrak{A}_{1}\right) \times \tau\left(\mathscr{A}_{2}\right)\right) \subset$ ᄃ $\tau\left(\hat{p}_{\mathrm{r}}\left(\mathfrak{A}_{1}\right)\right) \times \tau\left(\hat{p}_{2}\left(\mathcal{A}_{2}\right)\right)$.

Dimostrazione. - a) Sia $\mathrm{C}^{\prime}=\left\{C \in \sigma(\mathcal{C}): p^{-1}(C) \in \sigma\left(p^{-1}(\mathcal{C})\right)\right\}$. Allora $\mathrm{C}^{\prime}$ è una $\sigma$-algebra $\left(p^{-1}\left(\bigcup_{n \in N} C_{n}\right)=\bigcup_{n \in N} p^{-1}\left(C_{n}\right), p^{-1}(W \backslash C)=Z \backslash p^{-1}(C)\right.$ se $\left.C_{n}, C \in 2^{W}(n \in N)\right)$ che contiene $\mathfrak{C}$ e pertanto $\mathfrak{C}^{\prime}=\sigma(\mathfrak{C})$.

b) Sia $\mathbf{C}^{\prime}=\left\{C \in \tau(\mathcal{C}): p^{-1}(C) \in \tau\left(p^{-1}(\mathcal{C})\right)\right\}$. Allora $\quad \mathfrak{C}^{\prime} \quad \grave{\mathrm{e}}$ una topologia $\left(p^{-1}\left(\bigcup_{i \in I} C_{i}\right)=\bigcup_{i \in I} p^{-1}\left(C_{i}\right), p^{-1}\left(\bigcap_{j=0}^{k} D_{j}\right)=\bigcap_{j=0}^{k} p^{-1}\left(D_{j}\right)\right.$ se $\left.C_{i}, D_{j} \in \mathcal{2}^{W} \quad(i \in I, j \in\{0, \ldots, k\})\right)$ che contiene $\mathrm{C}$ e pertanto $\mathrm{e}^{\prime}=\tau(\mathcal{C})$.

c) Si ha che $p^{-1}(\hat{p}(\mathfrak{t})) \subset \mathcal{A t}$ : infatti se $C \in \hat{p}(\mathfrak{A})$ si ha che esiste $A \in \mathcal{A}$ tale che $C=p(A)$ e pertanto $p^{-1}(C)=p^{-1}(p(A))=A \in \mathcal{A}$. Allora da a) applicata a $\mathrm{C}=\hat{p}(\mathfrak{A})$ segue la prima parte della tesi. Sia ora $\mathcal{A}^{\prime}=\left\{A \in \sigma(\mathcal{A}): p^{-1}(p(A))=A\right\}$. Allora $\mathfrak{A}^{t}$ è una $\sigma$-algebra, poichè $p^{-1}\left(p\left(\bigcup_{n \in N} A_{n}\right)\right)=\bigcup_{n \in \mathbb{N}} p^{-1}\left(p\left(A_{n}\right)\right)$ se $A_{n} \in 2^{z} \quad(n \in N)$ e se $A \in \mathcal{A}^{\prime}$ risulta che $Z \backslash A=Z \backslash p^{-1}(p(A))$ e quindi $p(Z \backslash A)=p\left(Z \backslash p^{-1}(p(A))\right) \subset W \backslash p(A)$, da cui $Z \backslash A \subset p^{-1}(p(\mathbb{Z} \backslash A)) \subset p^{-1}(W \backslash p(A))=\mathscr{Z} \backslash p^{-1}(p(A))=\mathbb{Z} \backslash A$. Inoltre $\mathcal{A}^{\prime} \supset \mathcal{A}$ e quindi $\mathfrak{A}^{\prime} \supset \sigma(\mathcal{A})$, da cui $\mathfrak{A}^{\prime}=\sigma(\mathcal{A})$. Per provare ora la tesi basta dimostrare che, se $Z \in \mathcal{A}$ e se $\mathcal{A}^{\prime \prime}=\left\{A \in \sigma(\mathfrak{A}): p(\mathcal{A}) \in \sigma(\hat{p}(\mathcal{A})\}\right.$, allora $\mathcal{A}^{\prime \prime}=\sigma(\mathfrak{A})$. Si ha ovviamente 
che $\mathfrak{A}^{\prime \prime} \supset \mathfrak{A}$ e inoltre $\mathcal{A}^{\prime \prime}$ è una $\sigma$-algebra, visto che $p\left(\bigcup_{n \in N} A_{n}\right)=\bigcup_{n \in N} p\left(A_{n}\right)$ se $A_{n} \in 2^{Z}$ $(n \in N)$ e, se $A \in \mathcal{A}^{\prime \prime} \subset \sigma(\mathcal{A})=\mathcal{A}^{\prime}$, per quanto provato sopra risulta $p(Z \backslash A) \subset W \backslash p(A)$ e d'altra parte $p(Z \backslash A) \supset p(Z) \backslash p(A)$, per cui $p(Z \backslash A)=p(Z) \backslash p(A) \in \sigma(\hat{p}(\mathcal{A}))$ visto che $Z \in \mathcal{A}$. Pertanto $\mathcal{A}^{\prime \prime}=\sigma(\mathcal{A})$.

d) Come in $o$ ) si ha che $p^{-1}(\hat{p}(\mathfrak{A})) \subset \mathcal{A}$ e pertanto, applicando $\left.b\right)$ a $\mathcal{C}=\hat{p}(\mathfrak{A})$, si ottiene la prima parte della tesi. Come in $e)$, per concludere basta provare che $\mathcal{A}^{\prime}=\left\{A \in \tau(\mathfrak{A}): p^{-1}(p(A))=A\right\}$ e, nell'ipotesi che sia $Z \in \mathcal{A}, \mathfrak{A}^{\prime \prime}=\{A \in \tau(\mathfrak{A}): p(A) \in$ $\in \tau(\hat{p}(\mathfrak{A}))\}$ (che contengono ovviamente $\mathcal{A}$ ) sono topologie. Si ha che $p^{-1}\left(p\left(\bigcup_{i \in I} A_{i}\right)\right)=$ $=\bigcup_{i \in I} p^{-1}\left(p\left(A_{i}\right)\right), p\left(\bigcup_{i \in I} A_{i}\right)=\bigcup_{i \in I} p\left(A_{i}\right)$ se $A_{i} \in 2^{z}(i \in I)$ e che, se $E_{j} \in \mathcal{A}^{\prime}(j \in\{0, \ldots, k\})$, allora $\bigcap_{j=0}^{k} E_{j} \subset p^{-1}\left(p\left(\bigcap_{j=0}^{k} E_{j}\right)\right) \subset \bigcap_{j=0}^{k} p^{-1}\left(p\left(E_{j}\right)\right)=\bigcap_{j=0}^{k} E_{j} ;$ pertanto $\mathcal{A}^{\prime}=\tau(\mathcal{A})$ e quindi, se $E_{j} \in \mathcal{A}^{\prime \prime}(j \in\{0, \ldots, k\})$, allora $E_{j} \in \tau(\mathfrak{A})=\mathfrak{A}_{k}$, da cui $p\left(\bigcap_{j=0}^{k} E_{j}\right) \subset \bigcap_{j=0}^{l k} p\left(E_{j}\right)=$ $=p\left(p^{-1}\left(\bigcap_{j=0}^{k} p\left(E_{j}\right)\right)\right)=p\left(\bigcap_{j=0}^{k} p^{-1}\left(p\left(E_{j}\right)\right)\right)=p\left(\bigcap_{j=0}^{k} E_{j}\right)$ e, se $Z \in \mathcal{A}$, si ha che $p(Z) \in \hat{p}(\mathcal{A})$, per cui anche $\mathfrak{A}^{\prime \prime}$ è una topologia.

$e), f), g)$ Seguono rispettivamente dai seguenti fatti: $\left(p_{1} \times p_{2}\right)^{-1}\left(\left(p_{1} \times p_{2}\right)\left(A_{1} \times\right.\right.$ $\left.\left.\times A_{2}\right)\right)=\left(p_{1} \times p_{2}\right)^{-1}\left(p_{1}\left(A_{1}\right) \times p_{2}\left(A_{2}\right)\right)=p_{1}^{-1}\left(p_{1}\left(A_{1}\right)\right) \times p_{2}^{-1}\left(p_{2}\left(A_{2}\right)\right),\left(p_{1} \times p_{2}\right)^{-1}\left(C_{1} \times C_{2}\right)=$ $=p_{1}^{-1}\left(C_{1}\right) \times p_{2}^{-1}\left(C_{2}\right),\left(p_{1} \times p_{2}\right)\left(A_{1} \times A_{2}\right)=p_{1}\left(A_{1}\right) \times p_{2}\left(A_{2}\right)$ per ogni $A_{i} \in 2^{Z_{i}}, C_{i} \in 2^{W_{i}}(i=1,2)$.

h) Da $a)$ segue che $p_{i}^{-1}\left(\sigma\left(\mathrm{C}_{i}\right)\right) \subset \sigma\left(p_{i}^{-1}\left(\mathcal{C}_{i}\right)\right)(i=1,2)$ e quindi, utilizzando $\left.f\right)$ e poi di nuovo $a$ ), si conclude. ( $\mathrm{Si}$ noti che, se $W_{i} \in \mathrm{C}_{i}(i=1,2)$, la tesi si può provare anche usando $f$ ), $b$ ) del teorema 1.5 ed $a$ ) applicata a $\mathrm{C}=\left\{C_{1} \times C_{2}: C_{i} \in \mathcal{C}_{i}\right.$ $(i=1,2)\})$.

i) Da $b)$ segue che $p_{i}^{-1}\left(\tau\left(\mathcal{C}_{i}\right)\right) \subset \tau\left(p_{i}^{-1}\left(\mathcal{C}_{i}\right)\right)(i=1,2)$ e quindi, utilizzando $\left.f\right) \mathrm{e}$ poi di nuovo $b)$, si conclude.

j) Come in $c)$ si ha che $p_{i}^{-1}\left(\hat{p}_{i}\left(\mathfrak{A}_{i}\right)\right) \subset \mathfrak{A}_{i}(i=1,2)$ e pertanto, applicando $\left.h\right)$ a $\mathcal{C}_{i}=\hat{p}_{i}\left(\mathfrak{A}_{i}\right)(i=1,2)$, si ottiene la prima parte della tesi. Da $\left.c\right)$ segue inoltre che $p_{i}^{-1}\left(p_{i}(A)\right)=A$ per ogni $A \in \sigma\left(\mathcal{A}_{i}\right)(i=1,2)$ e quindi, utilizzando $\left.e\right)$ e di nuovo $\left.c\right)$, si ottiene la seconda parte della tesi. Infine, se $Z_{i} \in \mathcal{A}_{i}(i=1,2)$, usando $\left.\left.c\right), g\right)$ e di nuovo $c$ ) si conclude anche per quanto riguarda l'ultima parte della tesi. (Si noti che l'ultima parte della tesi si può provare anche utilizzando $b$ ) del teorema 1.5 e $c$ ) di questo lemma applicato con $\left.\mathcal{A}=\left\{A_{1} \times A_{2}: A_{i} \in \mathcal{A}_{i}(i=1,2)\right\}\right)$.

k) La prova è del tutto analoga a quella fatta in $j$ ). In questo caso basta applicare $i)$ a $\mathcal{C}_{i}=\hat{p}_{i}\left(\mathcal{A}_{i}\right)(i=1,2)$ per ottenere la prima parte della tesi; $\left.\left.d\right), e\right)$ e di nuovo $d$ ) per provare la seconda parte della tesi; $d$ ), $g$ ) e di nuovo $d$ ) per dimostrare la terza parte della tesi.

1.8 EsEMrr. - a) L'ultima parte di $c$ ) e di $d$ ) del lemma 1.7 può non valere se non si fa l'ipotesi che sia $p^{-1}(p(A))=A$ per ogni $A \in A$, Analogamente accade per l'ul- 
tima parte di $j$ ) e di $k$ ) del lemma 1.7 se non si fa l'ipotesi che sia $p_{i}^{-1}\left(p_{i}(A)\right)=A$ per ogni $A \in \mathcal{A}_{i}(i=1,2)$.

Basta infatti considerare $Z=\{a, b, c\}, W=\{d, e\}, p: Z \rightarrow W$ tale che $p(a)=$ $=p(c)=d, p(b)=e, \mathfrak{A}=\{\{a, b\},\{b, c\}, Z\}$. Allora $\hat{p}(\mathcal{A})=\{d, e\}$ e pertanto $\sigma(\hat{p}(\mathfrak{A}))=$ $=\tau(\hat{p}(\mathfrak{A}))=\{\emptyset, W\}$. D'altra parte $\{b\}=\{a, b\} \cap\{b, c\} \in \sigma(\mathfrak{A}) \cap \tau(\mathcal{A})$ e $p(\{b\})=$ $=\{e\} \notin\{\emptyset, W\}$.

Considerando ora $Z_{1}=\{f\}, W_{1}=\{g\}, p_{1}: Z_{1} \rightarrow W_{1}, p_{1}(f)=g, \mathfrak{t}_{1}=\left\{\emptyset, Z_{1}\right\}, Z_{2}, W_{2}$, $p_{2}, \mathcal{A}_{2}$ uguali rispettivamente a $Z, W, p, \mathfrak{A}$ di sopra, si ha che $\sigma\left(\hat{p}_{1}\left(\mathfrak{t}_{1}\right)\right) \times \sigma\left(\hat{p}_{2}\left(\mathfrak{t}_{2}\right)\right)=$ $=\tau\left(\hat{p}_{1}\left(\mathcal{A}_{1}\right)\right) \times \tau\left(\hat{p}_{2}\left(\mathcal{A}_{2}\right)\right)=\left\{\emptyset, W_{1} \times W_{2}\right\},\{(f, b)\}=\{(f, a),(f, b)\} \cap\{(f, b),(f, c)\} \in\left(\sigma\left(\mathcal{A}_{1}\right) \times\right.$ $\left.\times \sigma\left(\mathcal{A}_{2}\right)\right) \cap\left(\tau\left(\mathcal{A}_{1}\right) \times \tau\left(\mathcal{A}_{2}\right)\right)$ e $\left(p_{1} \times p_{2}\right)(\{(f, b)\})=\{(g, e)\} \notin\left\{\emptyset, W_{1} \times W_{2}\right\}$.

b) Per il caso delle $\sigma$-algebre si può dare anche un altro esempio della non validità delle ultime parti di $c$ ) e di $j$ ) del lemma 1.7 se non si fanno le ipotesi citate sopra.

Siano $(X, \varrho),(Y, \sigma)$ spazi topologici, $\varphi: X \rightarrow Y$ tale che $\varphi(A) \in \mathfrak{B}(\sigma)$ per ogni $A \in \mathfrak{B}(\varrho)$. Sia $\mathcal{L} \sigma$-algebra su $T$. Allora, come si vedrà nell'esempio che segue, non è detto che valga

$$
\left(i_{T} \times \varphi\right)(E) \in \mathfrak{L} \times \mathfrak{B}(\sigma) \quad \text { per ogni } E \in \mathfrak{L} \times \mathfrak{B}(\varrho)
$$

(e si ottiene un esempio per la non validità dell'ultima parte di $c$ ) del lemma 1.7, se non vale l'ipotesi che sia $p^{-1}(p(A))=A$ per ogni $A \in \mathcal{A}$, considerando $p=i_{T} \times \varphi$, $\mathfrak{A}=\mathfrak{L} \times \mathfrak{B}(\varrho)$ e utilizzando $g$ ) del lemma 1.7 ; un esempio per la non validità dell'ultima parte di $j$ ) del lemma 1.7 , se non vale l'ipotesi che sia $p_{i}^{-1}\left(p_{i}(A)\right)=A$ per ogni $A \in \mathcal{A}_{i}(i=1,2)$, considerando $\left.p_{1}=i_{T}, p_{2}=p, \mathfrak{A}_{1}=\mathcal{L}, \mathfrak{A}_{2}=\mathfrak{B}(\varrho)\right)$.

Si può notare inoltre che lo stesso esempio che ora si fornirà può essere utilizzato per far vedere che il teorema della proiezione (cfr. [7], Cap. III, Teorema 23) in generale non vale se lo spazio topologico considerato non è susliniano.

Si considerino $X=(\boldsymbol{R}, \delta), Y=(\boldsymbol{R}, \eta), T=\boldsymbol{R}, \mathfrak{L}=\mathfrak{L}(\boldsymbol{R}), \varphi: \boldsymbol{R} \rightarrow \boldsymbol{R}, \varphi(x)=\mathbf{0}$ per ogni $x \in \boldsymbol{R}$. Allora è ovvio che $\varphi(A) \in \mathfrak{B}(\eta)$ per ogni $A \in \mathfrak{B}(\delta)$. Sia inoltre $V \notin \mathscr{L}(\boldsymbol{R})$ (ad esempio $V$ l'insieme di Vitali su $[0,1])$, sia $\psi: V \rightarrow \boldsymbol{R}$ iniettiva e sia $D=\{(t, x) \in$ $\in \boldsymbol{R} \times \boldsymbol{R}: t \in V, x=\psi(t)\}$. Allora per ogni $x \in \boldsymbol{R}$ si ha che $D^{x}$ è $\emptyset$ oppure costituito da un solo punto e pertanto $D$ è chiuso in $\eta \times \delta$. Quindi per $a$ ) del teorema 1.5 risulta che $D \in \mathfrak{B}(\eta \times \delta) \subset \mathfrak{B}(\eta) \times \mathfrak{B}(\delta) \subset \mathcal{L}(\boldsymbol{R}) \times \mathfrak{B}(\delta)$. D'altra parte $\left(i_{T} \times \varphi\right)(D)=V \times\{0\} \notin$ $\notin \mathfrak{L}(\boldsymbol{R}) \times \mathfrak{B}(\eta)$, poichè se fosse $V \times\{0\} \in \mathfrak{L}(\boldsymbol{R}) \times \mathscr{B}(\eta)$ sarebbe $V \in \mathfrak{L}(\boldsymbol{R})$, il che è assurdo.

Si noti inoltre che $\operatorname{pr}_{T} D=\nabla \notin \mathfrak{L}(\boldsymbol{R})$ e quindi il teorema della proiezione non $\grave{\mathrm{e}}$ vero se si toglie l'ipotesi che $X$ sia separabile.

1.9 Teorema. - Siano $W, Z$ insiemi, $\varphi: W \rightarrow Z$. Allora:

a) se $\tau$ è una topologia su $W$ tale che $\sigma=\left\{\varphi^{-1}(\varphi(A)): A \in \tau\right\} \subset \tau$, si ha che $\sigma$ è una topologia su $W$; 
b) se $\varrho$ e $\tau$ sono topologie su. $W$ tali che $\varphi^{-1}(\varphi(A)) \in \varrho$ per ogni $A \in \tau$ e se $E \subset Z$ si ha che

$$
\varphi^{-1}(\varphi(A)) \in \varrho /_{\varphi^{-1}(E)} \quad \text { per ogni } A \in \tau /_{\varphi^{-2}(E)}
$$
Drmostrazione. - $-a)$ E ovvio che $\emptyset, W \in \sigma, \bigcup_{i \in I} B_{i} \in \sigma$ se $B_{i} \in \sigma\langle i \in I)$; se ora
$A_{i} \in \tau(i=0, \ldots, k)$ allora risulta che

$$
\bigcap_{i=0}^{k} \varphi^{-1}\left(\varphi\left(A_{i}\right)\right)=\varphi^{-1}\left(\varphi\left(\bigcap_{i=0}^{k} \varphi^{-1}\left(\varphi\left(A_{i}\right)\right)\right)\right)
$$

È ovvia l'inclusione del primo membro nel secondo perchè $C \subset \varphi^{-1}(\varphi(C))$ per $\underset{b}{\operatorname{ogni}} C \subset W$ e per quanto riguarda l'altra inclusione si ha che $\varphi^{-1}\left(\varphi\left(\bigcap_{i=0}^{k} \varphi^{-1}\left(\varphi\left(A_{i}\right)\right)\right)\right) \subset$ $c \bigcap_{i=0}^{k} \varphi^{-1}\left(\varphi\left(\varphi^{-1}\left(\varphi\left(A_{i}\right)\right)\right)\right)=\bigcap_{i=0}^{k} \varphi^{-1}\left(\varphi\left(A_{i}\right)\right)$. Pertanto vale $(1.9 .0)$ e, poichè $\varphi^{-1}\left(\varphi\left(A_{i}\right)\right) \in$ $\in \sigma \subset \tau$, si ha che $\bigcap_{i=0}^{h} \varphi^{-1}\left(\varphi\left(A_{i}\right)\right) \in \tau$ e si conclude.

b) Se $\left.A \in \tau\right|_{\varphi^{-1}(E)}$ allora esiste $A^{\prime} \in \tau$ tale che $A=A^{\prime} \cap \varphi^{-1}(E)$. Risulta ora che

$$
\varphi^{-1}\left(\varphi\left(A^{\prime} \cap \varphi^{-1}(E)\right)\right)=\varphi^{-1}\left(\varphi\left(A^{\prime}\right)\right) \cap \varphi^{-1}(E)
$$

Infatti $\varphi^{-1}\left(\varphi\left(A^{\prime} \cap \varphi^{-1}(E)\right)\right) \subset \varphi^{-1}\left(\varphi\left(A^{\prime}\right)\right) \cap \varphi^{-1}\left(\varphi\left(\varphi^{-1}(E)\right)=\varphi^{-1}\left(\varphi\left(A^{\prime}\right)\right) \cap \varphi^{-1}(E)=\right.$ $=\varphi^{-1}\left(\varphi\left(A^{\prime}\right) \cap E\right) \subset \varphi^{-1}\left(\varphi\left(A^{\prime} \cap \varphi^{-1}(E)\right)\right)$, ove per ottenere l'ultima inclusione si è tenuto conto del fatto che se $x \in \varphi\left(A^{\prime}\right) \cap E$ allora esiste $y \in A^{\prime}$ tale che $\varphi(y)=x$, da cui $y \in \varphi^{-1}(\{x\}) \subset \varphi^{-1}(E)$ e quindi $x \in \varphi\left(A^{\prime} \cap \varphi^{-1}(E)\right)$. Ora, visto che $\varphi^{-1}\left(\varphi\left(A^{\prime}\right)\right) \in \varrho$, da (1.9.1) segue che $\varphi^{-1}(\varphi(A))=\varphi^{-1}\left(\varphi\left(A^{\prime} \cap \varphi^{-1}(E)\right)\right) \in \varrho /_{\varphi^{-1}(E)}$.

1.10 Estmpio. - Se $W, Z, \varphi, \tau, \sigma$ sono come in $a)$ del teorema 1.9, $\sigma \subset \tau$, allora non è detto che sia

$$
\varphi^{-1}(\varphi(A)) \in s \sigma \quad \text { per ogni } A \in s \tau .
$$

Basta considerare $\varphi: R \rightarrow\{0,1\}$ tale che $\varphi(]-\infty, 0[)=\{0\}, \varphi([0, \infty[)=\{1\}$. Allora se $\tau=\{A \subset \boldsymbol{R}: \boldsymbol{R} \backslash A$ è al più numerabile $\} \cup\{\emptyset\}$, si ha ohe $\sigma=\{\boldsymbol{R}, \emptyset\}$ e quindi $s \sigma=\sigma$, mentre $s \tau=\delta$ (visto che in $\tau$ convergono solo le successioni definitivamente costanti) e allora se $A=\{0\}$ si ha che $A \in s \tau$, mentre $\varphi^{-1}(\varphi(A))=[0, \infty[\notin \sigma$.

1.11 Lemala. - Siano $(Z, \zeta)$ e $(W, \theta)$ spazi topologici, $\varphi: Z \rightarrow W$. Allora:

a) se $\varphi$ è aperta, se $\varphi^{-1}(\varphi(A))=A$ per ogni $A \in \zeta$ e se $\varphi(Z)$ è chiuso in $(W, \theta)$, si ha che $\varphi^{-1}(H)$ è compatto in $\zeta$ per ogni $H$ compatto in $\theta$; 
b) se $\varphi^{-1}(\varphi(A))=A$ per ogni $A \in \zeta$, si ha che $\varphi^{-1}(\varphi(K))$ è compatto in $\zeta$ per ogni $K$ compatto in $\zeta$;

o) se $\varphi_{Z, \varphi(Z)}:(Z, \zeta) \rightarrow(\varphi(Z), \theta / \varphi(Z))$ è aperta, se $\varphi^{-1}(\varphi(A))=A$ per ogni $A \in \zeta$, se $(I, \succ)$ è un insieme diretto, $z_{i}, z \in Z(i \in I), \lim _{i \in I} \varphi\left(z_{i}\right)=\varphi(z)$ in $\theta$, si ha che $\lim _{i \in I} z_{i}=z$ in $\zeta$;

d) se $\varphi_{Z, \varphi(Z)}:(Z, \zeta) \rightarrow\left(\varphi(Z), s\left(\left.\theta\right|_{\varphi(Z)}\right)\right)$ è aperta (cfr. definizione $\left.1.0 a\right)$ ), se $\varphi^{-1}(\varphi(A))=A$ per ogni $A \in \zeta$, se $z_{n}, z \in Z(n \in N), \lim _{n \rightarrow \infty} \varphi\left(z_{n}\right)=\varphi(z)$ in $\theta$, si ha che $\lim _{n \rightarrow \infty} z_{n}=z$ in $\zeta$.

Dimostrazione. - a) Sia $H$ compatto in $\theta$. Siano $A_{i} \in \zeta(i \in I)$ tali che $\bigcup A_{i} \supset$ $\supset \varphi^{-1}(H)$. Allora $\varphi\left(A_{i}\right) \in \theta \quad(i \in I)$ e $\bigcup_{i \in I} \varphi\left(A_{i}\right)=\varphi\left(\bigcup_{i \in I} A_{i}\right) \supset \varphi\left(\varphi^{-1}(H)\right)=H \cap \varphi(Z)$ e quindi $\left(\bigcup_{i \in I} \varphi\left(A_{i}\right)\right) \cup(W \backslash \varphi(Z)) \supset H$. Poichè $H$ è compatto e poichè $W \backslash \varphi(Z) \in \theta$, esistono $i_{1}, \ldots, i_{n} \in I$ tali che

$$
\left(\bigcup_{k=1}^{n} \varphi\left(A_{i_{k}}\right)\right) \cup(W \backslash \varphi(Z)) \supset H
$$

e allora

$$
\bigcup_{k=1}^{n} A_{i_{k}}=\varphi^{-1}\left(\varphi\left(\bigcup_{k=1}^{n} A_{i_{k}}\right)\right)=\varphi^{-1}\left(\bigcup_{k=1}^{n} \varphi\left(A_{i_{k}}\right)\right)=\varphi^{-1}\left[\left(\bigcup_{k=1}^{n} \varphi\left(A_{i_{k}}\right)\right) \cup(W \backslash \varphi(Z))\right] \supset \varphi^{-1}(H),
$$

ove per la prima eguaglianza si è tenuto conto del fatto che $\bigcup_{k=1}^{n} A_{i_{k}} \in \zeta$.

b) Sia $K$ compatto in $\zeta$. Siano $A_{i} \in \zeta(i \in I)$ tali che $\bigcup A_{i} \supset \varphi^{-1}(\varphi(K)) \supset \mathbb{K}$. Allora esistono $i_{1}, \ldots, i_{n} \in I$ tali che $\bigcup_{k=1}^{n} A_{i_{k}} \supset K$ e pertanto $\bigcup_{k=1}^{n} A_{i_{k}}=\varphi^{-1}\left(\varphi\left(\bigcup_{k=1}^{n} A_{i_{k}}\right)\right) \supset$
$\supset \varphi^{-1}(\varphi(K))$.

c) Siano $I, z_{i}, z(i \in I)$ come nell'enunciato. Sia $A \in \zeta$ tale che $z \in A$. Allora $\varphi(z) \in \varphi(A) \in \theta /_{\varphi(Z)}$, per cui esiste $i_{A} \in I$ tale che $\varphi\left(z_{i}\right) \in \varphi(A)$ per ogni $i \succ i_{A}$ e pertanto $z_{i} \in \varphi^{-1}\left(\left\{\varphi\left(z_{i}\right)\right\}\right) \subset \varphi^{-1}(\varphi(A))=A$ per ogni $i>i_{A}$ e quindi $\lim _{i \in I} z_{i}=z$ in $\zeta$.

d) Siano $z_{n}, z(n \in N)$ come nell'enunciato. Sia $A \in \zeta$ tale che $z \in A$. Allora $\varphi(z) \in \varphi(A) \in s(\theta / \varphi(Z))$ e d'altra parte dalle ipotesi segue che $\lim _{n \rightarrow \infty} \varphi\left(\mathcal{Z}_{n}\right)=\varphi(z)$ in $\theta / \varphi(Z)$ e da $a)$ del teorema 1.1 segue che $\lim _{n \rightarrow \infty} \varphi\left(z_{n}\right)=\varphi(z)$ in $s(\theta / \varphi(Z))$; pertanto esiste $n_{A} \in \boldsymbol{N}$ tale che $\varphi\left(z_{n}\right) \in \varphi(A)$ per ogni $n>n_{A}$. Quindi $z_{n} \in \varphi^{-1}\left(\left\{\varphi\left(z_{n}\right)\right\}\right) \subset \varphi^{-1}(\varphi(A))=A$ per ogni $n>n_{4}$, da cui $\lim _{n \rightarrow \infty} z_{n}=z$ in $\zeta$.

1.12 Defintzione. - Siano $\left(X_{i}, \varrho_{i}\right)$ spazi topologici $(i \in I), X$ insieme, $\varphi_{i}: X_{i} \rightarrow X$ $(i \in I)$. Allora si dice topologia finale delle topologie $\varrho_{i}$ rispetto alle $\varphi_{i}(i \in I)$ la topologia $\varrho$ su $X$ tale che $A \in \varrho$ se e solo se $\varphi_{i}^{-1}(A) \in \varrho_{i}$ per ogni $i \in I$.

Si ha anche che $Q$ è la più fine topologia su $X$ per cui per ogni $i \in I$ le $\varphi_{i}$ siano 
continue (cfr. [4], Cap. 1, §2, Proposizione 6). Se nel contesto ciò sarà sufficiente, si dirà semplicemente che $\varrho$ è la topologia finale per le $\phi_{i}(i \in I)$.

1.13 Definizione. - Siano $(X, \varrho)$ spazio topologico, $X_{j} \subset X(j \in J)$.

Si dice che $\left(\varrho,\left\{X_{j}: j \in J\right\}\right)$ verifica la proprietà $(L)$ se:

per ogni $x_{i}, x \in X(i \in I),(I, \succ)$ insieme diretto tali che $\lim _{i \in I} x_{i}=x$ in $\varrho$, segue che esistono $j \in J,(K, \cdot \succ)$ insieme diretto e $(\beta(k))_{k \in K}$ successione generalizzata estratta da $(i)_{i \in I}$ per cui $x, x_{\beta(k)} \in X_{j}$ per ogni $k \in K$.

Si dice che $\left(\varrho,\left\{X_{j}: j \in J\right\}\right)$ verifica la proprietà $(s L)$ se:

per ogni $x_{n}, x \in X(n \in N)$ tali che $\lim _{n \rightarrow \infty} x_{n}=x$ in $\varrho$, segue che esistono $j \in J$ e $\left(n_{k}\right)_{k \in N}$ successione strettamente crescente di naturali tali che $x, x_{n_{k}} \in X_{j}$ per ogni $k \in \boldsymbol{N}$.

1.14 Teorema. - Siano $(X, \varrho),\left(X_{j}, \varrho_{j}\right)$ spazi topologici, $\varphi_{j}: X_{j} \rightarrow X(j \in J)$. Allora:

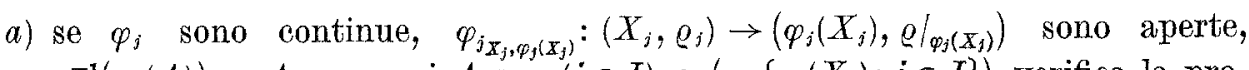
$\varphi_{j}^{-1}\left(\varphi_{j}(A)\right)=A$ per ogni $A \in \varrho_{j}(j \in J)$ e $\left(\varrho,\left\{\varphi_{j}\left(X_{j}\right): j \in J\right\}\right)$ verifica la proprietà $(\mathrm{L})$, si ha che $\varrho$ è la topologia finale delle topologie $\varrho_{j}$ rispetto alle $\varphi_{j}(j \in J)$;

b) se $\varphi_{j}$ sono sequenzialmente continue, $\varphi_{j_{X_{j}, \varphi_{j}\left(X_{j}\right)}}:\left(X_{j}, \varrho_{j}\right) \rightarrow\left(\varphi_{j}\left(X_{j}\right), s\left(\left.\varrho\right|_{\varphi_{j}\left(X_{j}\right)}\right)\right)$ sono aperte, $\varphi_{i}^{-1}\left(\varphi_{j}(A)\right)=A$ per ogni $A \in \varrho_{j}(j \in J)$ e $\left(\varrho,\left\{\varphi_{j}\left(X_{j}\right): j \in J\right\}\right)$ verifica la proprietà $(\mathrm{sL})$, si ha che $8 \varrho$ è la topologia finale delle topologie so, rispetto alle $\varphi_{j}(j \in J)$.

Dimostrazione. - Dalla continuità [risp. sequenziale continuità] delle $\varphi_{j}(j \in J)$ segue che $\varrho$ [risp. s $\varrho$ ] è meno fine della topologia finale delle topologie $\varrho_{j}$ [risp. $s \varrho_{j}$ ] rispetto alle $\varphi_{j}(j \in J)$. Esista ora per assurdo $A \notin \varrho[$ risp. $A \notin s \varrho]$ tale che $\varphi_{j}^{-1}(A) \in \varrho_{j}$ [risp. $\left.\varphi_{j}^{-1}(A) \in s Q_{j}\right]$ per ogni $j \in J$. Allora esistono $x \in A,(I, \succ)$ insieme diretto e $x_{i} \in X \backslash A(i \in I)$ tali che $\lim _{i \in I} x_{i}=x$ in 0 [risp. esistono $x \in A$ e $x_{n} \in X \backslash A(n \in N)$ tali che $\lim _{n \rightarrow \infty} x_{n}=x$ in $\left.\varrho\right]$. Pertanto per la proprietà (L) [risp. (sL)] esistono $j \in J$, $(K, \bullet \succ)$ insieme diretto e $(\beta(k))_{k \in K}$ successione generalizzata estratta da $(i)_{i \in I}$ [risp. $j \in J,\left(n_{k}\right)_{k \in N}$ successione strettamente crescente di naturali] tali che $x, x_{\beta(k)} \in \varphi_{j}\left(X_{j}\right)$ [risp. $\left.x, x_{m_{k}} \in \varphi_{j}\left(X_{j}\right)\right]$ per ogni $k \in K\left[\right.$ risp. $k \in N$ ]. Dunque esistono $y, y_{\beta(k)} \in X_{j}$ [risp. $\left.y, y_{n_{k}} \in X_{j}\right]$ tali che $\varphi_{j}(y)=x, \varphi_{j}\left(y_{\beta(k)}\right)=x_{\beta(k)}\left[\operatorname{risp} . \varphi_{j}(y)=x, \varphi_{j}\left(y_{n_{k}}\right)=x_{n_{k}}\right.$ ] e per $\left.c\right)$ [risp. d)] del lemma 1.11 risulta che $\lim _{k \in K} y_{\beta(k)}=y$ in $\varrho_{j}$ [risp. $\lim _{k \rightarrow \infty} y_{n_{k}}=y$ in $\varrho_{j}$ e quindi in $s \varrho_{j}$ per a) del teorema 1.1]. D'altra parte $y \in \varphi_{j}^{-1}(\{x\}) \subset \varphi_{j}^{-1}(A)$, che è in $\varrho_{j}$ [risp. in $s \varrho_{j}$ ] e quindi esiste $k_{A} \in K$ [risp. $\left.k_{A} \in N\right]$ tale che $y_{\beta(k)} \in \varphi_{j}^{-1}(A)$ se $k \cdot>k_{A}$ [risp. $y_{n_{k}} \in \varphi_{j}^{-1}(\boldsymbol{A})$ se $\left.k>k_{A}\right]$. Pertanto $x_{\beta(k)}=\varphi_{j}\left(y_{\beta(k)}\right) \in A \quad\left[\operatorname{risp} . x_{n_{k}}=\varphi_{j}\left(y_{n_{k}}\right) \in A\right]$, il che è assurdo. 
1.15 Esenpr. - a) Si noti che nelle ipotesi di a) del teorema 1.14, se si sostituisce l'ipotesi sulla proprietà (L) con la stessa ipotesi sulla proprietà (sL), la tesi può non valere, anche se $J=N, X_{N} \subset X, \varphi_{N}: X_{N} \rightarrow X$ le immersioni, $\varrho_{N}=\varrho / x_{N}, X_{N} \subset X_{N+1}$ e $X_{N}$ chiuso in $(X, \varrho)$ per ogni $N \in N$.

Basta infatti considerare $X$ spazio di Hilbert separabile e di dimensione infinita su $\boldsymbol{R}, \varrho=w(X),\left\{e_{n}: n \in N\right\}$ un sistema O.N.C. in $X, X_{N}=\overline{S_{X}(0, N)}(N \in N)$. Si ha che $\left(w(X),\left\{\overline{S_{X}(0, N)}: N \in \boldsymbol{N}\right\}\right)$ verifica la proprietà (sL) perchè le successioni convergenti in $w(X)$ sono limitate. Sia ora $A=X \backslash\left\{\sqrt{n+1} e_{n}: n \in N\right\}$. Allora $0 \in A \backslash \AA$,

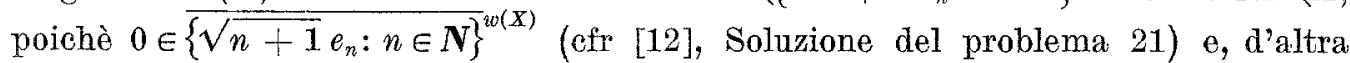
parte $\left(\varphi_{N}\right)^{-1}(A)=A \cap \overline{S_{X}(0, N)}=\overline{S_{X}(0, N)} \backslash\left\{\sqrt{n+1} e_{n}: n+1 \leqslant N^{2}\right\} \in w(X) / \overline{S_{x}(0, N)}$, visto che $\left\{\sqrt{n+1} e_{n}: n \leqslant N^{2}-1\right\}$ è finito e quindi chiuso in $\overline{S_{X}(0, N)}(N \in N)$.

$\left.b^{\prime}\right)$ Si noti che, se $(X, \varrho),\left(X_{j}, \varrho_{j}\right)$ sono spazi topologici, $\varphi_{j}: X_{j} \rightarrow X(j \in J)$ sono tali che $\varrho$ sia la topologia finale delle topologie $\varrho_{j}$ rispetto alle $\varphi_{j}(j \in J)$, $\varphi_{j_{X_{j}}, \varphi_{j}\left(X_{j}\right)}:\left(X_{j}, \varrho_{j}\right) \rightarrow\left(\varphi_{j}\left(X_{j}\right), \varrho / \varphi_{\varphi_{j}\left(X_{j}\right)}\right)$ sono aperte, $\varphi_{j}^{-1}\left(\varphi_{j}(A)\right)=A$ per ogni $A \in \varrho_{j}$ $(j \in J), \bigcup_{j \in J} \varphi_{j}\left(X_{j}\right)=X$, non è detto che $\left(\varrho,\left\{\varphi_{j}\left(X_{j}\right): j \in J\right\}\right)$ verifichi la proprietà $(\mathrm{L}) \mathrm{e}$ nemmeno la proprietà (sL). Si noti inoltre che nell'esempio che segue $\varrho$ e $\varrho_{j}$ sono a base numerabile di aperti $(j \in J)$ e che quindi per $b$ ) del teorema 1.1 in tale esempio si ha: $s \varrho=\varrho, s \varrho_{j}=\varrho_{j}(j \in J)$.

Basta considerare $X=N, J=N, X_{N}=\{0, \ldots, N\}(N \in N), \varphi_{N}: X_{N} \rightarrow X$ le immersioni, $\varrho_{N}=\{[M, N] \cap\{0, \ldots, N\}: M \in N, M \leqslant N\} \cup\{\emptyset\}$. Pertanto $\varrho=\{[M, \infty[\cap$ $\cap \boldsymbol{N}: M \in \boldsymbol{N}\}$ e $(n)_{n \in N}$ è una successione che converge a qualunque elemento di $\boldsymbol{N}$ (ad esempio 0), ma che non ha alcuna estratta (neanche generalizzata) che sia limitata.

$\left.b^{\prime \prime}\right)$ Si può fare anche un altro esempio in cui, nelle condizioni di $\left.b^{\prime}\right),\left(\varrho,\left\{\varphi_{j}\left(X_{j}\right)\right.\right.$ : $j \in J\})$ non verifica la proprietà (L) anche se $\varrho$ e $\varrho_{j}(j \in J)$ sono $T_{2}$.

Basta considerare $x_{0} \notin \boldsymbol{R} \cup \boldsymbol{R}^{2}, X_{0}=\boldsymbol{R} \cup\left\{x_{0}\right\}$ e $\varrho_{0}$ tale che $\left(X_{0}, \varrho_{0}\right)$ sia la compattificazione con un punto di $(\boldsymbol{R}, \eta), X_{\mathbf{1}}=\boldsymbol{R}^{2}, \varrho_{1}=\eta \times \eta, X=\boldsymbol{R}^{\mathbf{2}} \cup\left\{x_{0}\right\}, \varphi_{j}: X_{j} \rightarrow X$ $(j \in\{0,1\})$ tali che $\varphi_{0}(x)=(x, 0)$ per ogni $s \in \boldsymbol{R}, \varphi_{0}\left(x_{0}\right)=x_{0}, \varphi_{1}$ l'immersione. Allora $\varrho=(\eta \times \eta) \cup\left\{\left\{x_{0}\right\} \cup A: A \in \eta \times \eta, A \supset(]-\infty, a[\cup] b, \infty[) \times\{0\}\right.$ per qualche $\left.a, b \in \boldsymbol{R}\right\}$. Pertanto, se si considera l'insieme diretto $(\mathcal{U}, \succ)$, ove $\mathcal{U}=\left\{U\right.$ intorni di $x_{0}$ in $\left.\varrho\right\}$ e $U_{1} \succ U_{2}\left(U_{1}, U_{2} \in \mathcal{U}\right)$ se $U_{1} \subset U_{2}$, per ogni $U \in \mathcal{U}$ esiste $y_{U} \in U$ tale che $y_{U} \notin \varphi\left(X_{0}\right)$. Quindi $\lim _{U \in \mathcal{U}} y_{U}=x_{0}$ in $\varrho$ ma per nessuna estratta $(\beta(k))_{k \in K}$ di $(U)_{U \in \mathcal{U}}(\operatorname{con}(K, \cdot>)$ insieme diretto) si può avere che $y_{\beta(k)} \in \varphi_{0}\left(X_{0}\right)$ per ogni $k \in K$.

1.16 Teorema. - Siano $(X, \varrho)$ spazio topologico, $X_{j} \subset X(j \in J)$. Allora:

a) se $\left(\varrho,\left\{X_{j}: j \in J\right\}\right)$ verifica la proprietà $(L)$, si ha che $\left(\varrho,\left\{X_{j}: j \in J\right\}\right)$ verifica la proprietà $(\mathrm{sL})$;

b) se $\left(\varrho,\left\{X_{j}: j \in J\right\}\right)$ verifica la proprietà $(\mathrm{L})$, si ha che $\left(8 \varrho,\left\{X_{j}: j \in J\right\}\right)$ verifica la proprietà $(\mathrm{L})$; 
c) se $\left(s \varrho,\left\{X_{j}: j \in J\right\}\right)$ verifica la proprietà $(L)$, si ha che $\left(s \varrho,\left\{X_{j}: j \in J\right\}\right)$ verifica la proprietà (sL);

d) si ha che $\left(\varrho,\left\{X_{j}: j \in J\right\}\right)$ verifica la proprietà (sL) se e solo se $\left(s \varrho,\left\{X_{j}: j \in J\right\}\right)$ verifica la proprietà (sL).

Dimostrazione. - a) Siano $x_{n}, x \in X(n \in N)$ tali che $\lim _{n \rightarrow \infty} x_{n}=x$ in $\underline{o}$. Allora per l'ipotesi esistono $j \in J,(K, \succ)$ insieme diretto e $(\beta(k))_{k \in K}$ successione generalizzata estratta da $(n)_{n \in \boldsymbol{N}}$ tali che $x, x_{\beta(k)} \in X_{j}$ per ogni $k \in K$. Pertanto esistono $k_{0} \in K$ tale che $\beta\left(k_{0}\right)>0$ e $k_{1} \in K$ tale che $\beta\left(k_{1}\right)>\max \left(1, \beta\left(k_{0}\right)\right)$ e, se $h \in N \backslash\{0\}$ e $k_{m} \in K$ sono tali che $\beta\left(k_{m}\right)>\max \left(m, \beta\left(k_{m_{-1}}\right)\right)(m \in\{1, \ldots, h\})$, esiste $k_{h_{+1}} \in K$ tale che $\beta\left(k_{h_{+1}}\right)>$ $>\max \left(h+1, \beta\left(k_{h}\right)\right)$. Considerando ora $n_{h}=\beta\left(k_{h}\right)$ per ogni $h \in N$, si ha che $\left(n_{h}\right)_{h \in N}$ è una successione strettamente crescente di naturali tale che $x, x_{n_{h}} \in X_{j}$ per ogni $h \in \mathbf{N}$.

b) Basta osservare che, per $b)$ del teorema 1.1, se $x_{i}, x \in X(i \in I)$, con $(I, \succ)$ insieme diretto, sono tali che $\lim _{i \in I} x_{i}=x$ in $s \varrho$, allora risulta che $\lim _{i \in I} x_{i}=x$ in $\varrho \mathrm{e}$ utilizzare l'ipotesi.

c) Segue da a) applicato alla topologia s@.

d) Basta tener conto di come è definita la proprietà (sL) e del fatto che per a) del teorema 1.1 , se $x_{n}, x \in X(n \in N)$, si ha che $\lim _{n \rightarrow \infty} x_{n}=x$ in $\varrho$ se e solo se $\lim _{n \rightarrow \infty} x_{n}=x$ in $s \varrho$.

1.17 EsEMPI. - Siano $(X, \varrho)$ spazio topologico, $X_{j} \subset X(j \in J)$. Allora:

a) non è detto che, se $\left(\varrho,\left\{X_{j}: j \in J\right\}\right)$ verifica la proprietà (sL), si abbia che $\left(s \varrho,\left\{X_{i}: j \in J\right\}\right)$ verifichi la proprietà $(L)$;

b) non è detto che, se $\left(s \varrho,\left\{X_{j}: j \in J\right\}\right)$ verifica la proprietà $(\mathrm{L})$, si abbia che $\left(\varrho,\left\{X_{j}: j \in J\right\}\right)$ verifichi la proprietà $(\mathrm{L})$.

Basta considerare rispettivamente i due seguenti esempi.

a) Siano $X$ spazio di Hilbert separabile e di dimensione infinita su $\boldsymbol{R}, \varrho=w(X)$, $X_{j}=S_{X}(0, j)\left(j \in \boldsymbol{Z}_{+}\right)$. Allora se $\lim _{n \rightarrow \infty} x_{n}=x$ in $w(X)$ si ha che $\left(x_{n}\right)_{n \in N}$ è limitata, pertanto esiste $j \in \boldsymbol{Z}_{+}$tale che $x, x_{n} \in S_{X}(\mathbf{0}, j)$ per ogni $n \in \boldsymbol{N}$ e quindi $\left(w(X),\left\{S_{X}(0\right.\right.$, $\left.j): j \in \boldsymbol{Z}_{+}\right\}$) verifica la proprietà (sL). D'altra parte, se $k \in \boldsymbol{R}$ e se $\left\{e_{n}: n \in \boldsymbol{N}\right\}$ è un sistema O.N.C. in $X$, si ha che $\lim _{n \rightarrow \infty} k e_{n}=0$ in $w(X)$ e quindi, per $\left.a\right)$ del teorema 1.1, anche in $s w(X)$; pertanto 0 non ha intorni limitati in $s w(X)$. Se ora si considera $(\mathcal{\vartheta}, \succ)$ l'insieme diretto tale che $V=\{(U, n): U$ intorno di 0 in $s w(X), n \in N\}$ e con $\left(U_{1}, n_{1}\right) \succ\left(U_{2}, n_{2}\right)\left(\left(U_{1}, n_{1}\right),\left(U_{2}, n_{2}\right) \in \mathcal{U}\right)$ se e solo se $U_{1} \subset U_{2}$ e $n_{1}>n_{2}$, se per ogni $(U, n) \in \mathcal{V}$ si considera $x_{U, n} \in U,\left|x_{U, n}\right|_{X}>n$, si ha che $\lim _{(U, n) \in \mathcal{U}} x_{U, n}=0$ in $\operatorname{sw}(X) \mathrm{e}$ non esiste alcuna successione generalizzata estratta da $\left(x_{U, n}\right)_{(U, n) \in \mathcal{V}}$ e costituita da 
elementi appartenenti ad una sfera di $X$. Pertanto $\left(s w(X),\left\{S_{\Sigma}(0, j): j \in Z_{+}\right\}\right)$non verifica la proprietà $(\mathrm{L})$.

b) Siano $X$ spazio di Hilbert separabile e di dimensione infinita su $\boldsymbol{R}, \varrho=$ $=w(X),\left\{e_{n}: n \in \boldsymbol{N}\right\}$ un sistema O.N.C. in $X$. Allora, poichè per l'esempio $1.2 a$ ) risulta che $0 \in\left\{\sqrt{n+1} e_{n}: n \in N\right\}^{w(X)}$ e che $\left\{\sqrt{n+1} e_{n}: n \in N\right\}$ è sequenzialmente chiuso in $w(X)$ (visto che le successioni ad elementi in $\left\{\sqrt{n+1} e_{n}: n \in N\right\}$ e convergenti in $w(X)$ sono definitivamente costanti), si ha che

$$
0 \in\left\{{\left.\overline{\sqrt{n+1}} e_{n}: n \in N, n \geqslant j\right\}^{w(X)}}^{w(x)} \text { ogni } j \in N\right.
$$

(visto che $\left\{\sqrt{n+1} e_{n}: n \in N, n<j\right\}$ è chiuso in $w(X)$ per ogni $j \in N$ ) e che

$$
\left\{\sqrt{n+1} e_{n}: n \in N, n \geqslant j\right\} \quad \text { è sequenzialmente chiuso in } w(X)(j \in N) \text {. }
$$

Quindi, considerando $X_{j}=X \backslash\left\{\sqrt{n+1} e_{n}: n \in N, n \geqslant j\right\} \quad(j \in N)$, si ha che se $x_{i}$, $x \in X(i \in I)$, con $(I, \succ)$ insieme diretto, sono tali che $\lim _{i \in I} x_{i}=x$ in $s w(X)$, poichè $\bigcup_{j \in N} X_{j}=X$ esiste $j \in N$ tale che $x \in X_{j}$ e, poichè $X_{j} \in s w(X)$, esiste $i_{j} \in I$ tale che $x_{i} \in X_{j}$ per ogni $i \succ i_{j}$, per cui $\left(s w(X),\left\{X_{j}: j \in N\right\}\right)$ verifica la proprietà (L). D'altra parte, se $(V, \cdot>)$ è l'insieme diretto tale che $\vartheta=\{(U, j): U$ intorno di 0 in $w(X)$, $j \in N\}$ e con $\left(U_{1}, j_{1}\right) \cdot>\left(U_{2}, j_{2}\right)\left(\left(U_{1}, j_{1}\right),\left(U_{2}, j_{2}\right) \in \mathcal{U}\right)$ se e solo se $U_{1} \subset U_{2}$ e $j_{1}>j_{2}$, allora per ogni $(U, j) \in \mathcal{V}$, visto che $0 \in\left\{\sqrt{\sqrt{n+1}} e_{n}: n \in N, n \geqslant j\right\}^{w(X)}$, esiste $x_{U, j} \in$ $\in\left\{\sqrt{n+1} e_{n}: n \in N, n \geqslant j\right\} \cap U$. Pertanto $\lim _{0, j} x_{U, j}=0$ in $w(X)$ e non esiste alcuna successione generalizzata estratta da $\left(x_{U, j}\right)_{(U, j) \in} \mathcal{V}$ e costituita da elementi appartenenti ad un solo insieme $X_{j}$ (per qualche $j \in N$ ). Quindi $\left(w(X),\left\{X_{j}: j \in N\right\}\right.$ ) non verifica la proprietà $(\mathrm{L})$.

1.18 Trortima. - Siano $(X, \varrho),(Y, \sigma)$ spazi topologici, $X_{i, j} \subset X, Y_{j} \subset Y\left(i \in I_{j}\right.$, $j \in J),\left(\varrho,\left\{X_{i, l}: i \in I_{l}\right\}\right)$ e $\left(\sigma,\left\{Y_{j}: j \in J\right\}\right)$ che verificano la proprietà (L) [risp. (sL)] per ogni $l \in J$. Allora $\left(\varrho \times \sigma,\left\{X_{i, j} \times Y_{3}: i \in I_{i}, j \in J\right\}\right)$ verifica la proprietà (L) [risp. $(\mathrm{sL})]$.

Dimostrazione. - Se $\left(x_{\alpha}, y_{\alpha}\right),(x, y) \in X \times Y \quad(\alpha \in A,(A, \succ)$ insieme diretto), $\lim _{\alpha \in A}\left(x_{\alpha}, y_{\alpha}\right)=(x, y)$ in $\varrho \times \sigma$ [risp. se $\left(x_{n}, y_{n}\right),(x, y) \in X \times Y(n \in N), \lim _{n \rightarrow \infty}\left(x_{n}, y_{n}\right)=$ $=(x, y)$ in $\varrho \times \sigma]$, allora esistono $j \in J,(H, \cdot \succ)$ insieme diretto e $(\gamma(h))_{h \in H}$ successione generalizzata estratta da $(\alpha)_{\alpha \in A}$ [risp. $j \in J$ e $\left(n_{h}\right)_{h \in N}$ successione strettamente crescente di naturali] tali che $y_{\gamma(h)}, y \in Y_{j}\left[\right.$ risp. $y_{n_{h}}, y \in Y_{j}$ ] per ogni $h \in H$ [risp. $\left.h \in N\right] \mathrm{e}$ quindi esistono $i \in I_{j},(K, \cdots \succ)$ insieme diretto e $(\beta(k))_{k \in K}$ successione generalizzata estratta da $(\gamma(h))_{h \in H}\left[\right.$ risp. $i \in I_{j}$ e $\left(h_{k}\right)_{k \in N}$ successione strettamente crescente di naturali] tali che $x_{\beta(k)}, x \in X_{i, j}$ [risp. $x_{n_{h_{i b}}}, x \in X_{i, j}$ ] per ogni $k \in K$ [risp. $k \in N$ ]. Pertanto $\left(x_{\beta(k)}, y_{\beta(k)}\right),(x, y) \in X_{i, j} \times Y_{j}\left[\right.$ risp. $\left.\left(x_{n_{h_{k}}}, y_{n_{k_{k}}}\right),(x, y) \in X_{i, j} \times Y_{j}\right]$ per ogni $T_{i} \in K$ [risp. $k \in N]$. 
1.19 TEorema. - Siano $(X, \varrho),\left(X_{j}, \varrho_{j}\right)$ spazi topologici, $\varphi_{j}: X_{j} \rightarrow X(j \in J), \varrho$ topologia finale delle topologie $\varrho_{j}$ rispetto alle $\varphi_{j}(j \in J)$. Allora:

a) se $\varrho_{j}=s \varrho_{j}$ per ogni $j \in J$, risulta che $\varrho=8 \varrho$;

b) se $\sigma$ è la topologia finale delle topologie $s \varrho_{j}$ rispetto alle $\varphi_{j}(j \in J)$, si ha che $\sigma \supset s Q ;$

c) se $\sigma$ ̀̀ come in $b)$, se per ogni $j \in J$ si ha che $\varphi_{i_{X_{j}, \varphi_{j}\left(X_{j}\right)}}:\left(X_{j}, \underline{o}_{j}\right) \rightarrow\left(\varphi_{j}\left(X_{j}\right)\right.$, $\left.s\left(\varrho /_{\varphi_{j}\left(X_{j}\right)}\right)\right)$ è aperta, $\varphi_{j}^{-1}\left(\varphi_{j}(A)\right)=A$ per ogni $A \in \varrho_{j}$ e se $\left(\varrho / \cup_{j \in J} \varphi_{j}\left(X_{j}\right)\right.$, $\left.\left\{\varphi_{j}\left(X_{j}\right): j \in J\right\}\right)$ verifica la proprietà (sL), risulta che $\sigma=s \varrho$.

Dimostrazione. - a) Per $b$ ) del teorema 1.1 e per la definizione $1.0 a$ ), basta provare che gli insiemi sequenzialmente chiusi in $\varrho$ sono chiusi in $\varrho$. Sia $C$ sequenzialmente chiuso in $\varrho$. Allora $\varphi_{i}^{-1}(C)$ è sequenzialmente chiuso in $\varrho_{j}$ poichè $\varphi_{j}:\left(X_{j}, \varrho_{j}\right) \rightarrow$ $\rightarrow(X, \varrho)$ è continua e per $f)$ del teorema $1.1(j \in J)$. Pertanto, visto che $\varrho_{j}=s \varrho_{j}$ $(j \in J)$, si ha che $\varphi_{j}^{-1}(C)$ è chiuso in $\varrho_{j}$ per ogni $j \in J$. Ne segue che $C$ è chiuso in $\varrho$.

b) Poichè per $c$ ) del teorema 1.1 risulta che $s\left(s \varrho_{j}\right)=s \varrho_{j}(j \in J)$, da $\left.a\right)$ segue che $\sigma=s \sigma$ e, poichè $s \varrho_{j} \supset \varrho_{j}(j \in J)$ per $\left.b\right)$ del teorema 1.1, si ha anche che $\sigma \supset \varrho$ Allora $\sigma=s \sigma \supset s \varrho$.

c) Poichè per $b$ ) si ha che $\sigma \supset s \varrho$, per $a$ ) del teorema 1.1 basta provare che se $x_{n}, x \in X(n \in N), \lim _{n \rightarrow \infty} x_{n}=x$ in $\varrho$ allora $\lim _{n \rightarrow \infty} x_{n}=x$ in $\sigma$. Siano ora $x_{n}, x \in X(n \in N)$, $\lim _{n \rightarrow \infty} x_{n}=x$ in $\varrho$. Se $x \notin \bigcup_{j \in J} \varphi_{j}\left(X_{j}\right)$ allora $\{x\} \in \varrho$ e pertanto $\left(x_{n}\right)_{n \in N}$ è definitivamente costante e uguale a $x$ e quindi convergente a $x$ in $\sigma$. Se $x \in \bigcup_{j \in J} \varphi_{j}\left(X_{j}\right)$, visto che $\bigcup_{j \in J} \varphi_{j}\left(X_{j}\right) \in \varrho$, esiste $n_{0} \in N$ tale che $x_{n} \in \bigcup_{j \in J} \varphi_{j}\left(X_{j}\right)$ per ogni $n>n_{0}$. Quindi per ogni estratta di $\left(x_{n}\right)_{n \in N}$ esistono $j \in J$ e una sottosuccessione $\left(x_{n_{k}}\right)_{k \in N}$ tali che $x$, $x_{n_{k}} \in \varphi_{j}\left(X_{j}\right)$ per ogni $k \in N$. Se ora $y, y_{k} \in X_{j}$ sono tali che $\varphi_{j}(y)=x, \varphi_{j}\left(y_{k}\right)=x_{n_{k}}$ $(k \in N)$, per $d)$ del lemma 1.11 si ha che $\lim _{k \rightarrow \infty} y_{k}=y$ in $\varrho_{j}$. Pertanto per $\left.a\right)$ del teorema 1.1 risulta che $\lim _{k \rightarrow \infty} y_{k}=y$ in $s Q_{j}$ e quindi $x_{n_{k}}=\varphi_{j}\left(y_{k}\right) \rightarrow \varphi_{j}(y)=x$ in $\sigma$ poichè $\varphi_{j}:\left(X_{j}, s \varrho_{j}\right) \rightarrow(X, \sigma)$ è continua. Perciò $\lim _{n \rightarrow \infty} x_{n}=x$ in $\sigma$.

1.20 EsaMPIo. - Nelle ipotesi del teorema $1.19 b)$ può accadere che $\sigma \supsetneqq s \varrho$. Siano $X=X_{0}=X_{1}=\boldsymbol{R}, \varphi_{0}=\varphi_{1}=i_{\boldsymbol{R}}, \varrho_{0}=\{A$ intorni euclidei di 0$\} \cup\{A \backslash\{0\}: A$ intorno euclideo di 0$\} \cup\{\{0\}, \emptyset\}, \varrho_{1}=\{A \subset \boldsymbol{R}: 0 \notin A\} \cup\{A \subset \boldsymbol{R}: \boldsymbol{R} \backslash A$ è al più numerabile $\}$. Allora $\varrho_{0}$ è a base numerabile di intorni e quindi $s \varrho_{0}=\varrho_{0}$ per $b$ ) del teorema 1.1; in $\varrho_{1}$, che è $T_{2}$, convergono solo le successioni definitivamente costanti e quindi $s \varrho_{1}=\delta$. Pertanto $\sigma=\varrho_{0}$. Inoltre $\varrho=\{A \backslash\{0\}: A$ intorno euclideo d i 0$\} \cup\{A: A$ intorno euclideo di $0, \boldsymbol{R} \backslash A$ al più numerabile $\} \cup\{\emptyset\}$. Per provare ora che $s \varrho \subsetneq \sigma$, visto che $\sigma=s \sigma$, visto che $s \varrho \subset \sigma$ per $b$ ) del teorema 1.19 e visto che per $a$ ) del teorema 1.1 una successione è convergente in una topologia $\tau$ se e solo se è convergente 
in $s \tau$, basta dimostrare che esiste $\left(x_{n}\right)_{n \in N}$ tale che $x_{n} \in \boldsymbol{R}(n \in \boldsymbol{N}), x_{n} \rightarrow x \in \boldsymbol{R}$ in $\varrho$, $x_{n}$ non tende a $x$ in $\sigma$. Quindi si conclude osservando che $1 /(n+1) \rightarrow 0$ in $\varrho, 1 /(n+1)$ non tende a 0 in $\sigma$.

Si noti che l'esempio considerato sopra verifica tutte le ipotesi di $e$ ) del teorema 1.19 , tranne il fatto che $\left.\varphi_{j_{X_{j}, \varphi_{j}\left(X_{j}\right)}}:\left(X_{j}, \varrho_{j}\right) \rightarrow\left(\varphi_{j}\left(X_{j}\right), s\left(\left.\varrho\right|_{\varphi_{j}\left(X_{j}\right)}\right)\right)\right)$ siano aperte $(j \in J)$.

1.21 Teorema. - Siano $\left(X_{j}, \varrho_{j}\right),\left(Y_{i}, \sigma_{i}\right)$ spazi topologici, $X, Y$ insiemi, $\varphi_{j}: X_{j} \rightarrow X$, $\psi_{i}: Y_{i} \rightarrow Y(j \in J, i \in I)$. Siano inoltre $\varrho$ [risp. $\varrho^{s}$ ] la topologia finale delle topologie $o_{j}$ [risp. $\left.s \varrho_{j}\right]$ rispetto alle $\varphi_{j}(j \in J), \sigma$ [risp. $\left.\sigma^{s}\right]$ la topologia finale delle topologie $\sigma_{i}$ [risp. $s \sigma_{i}$ ] rispetto alle $\psi_{i}(i \in I)$ e $\tau$ [risp. $\left.\tau^{s}\right]$ la topologia finale delle topologie $\varrho_{j} \times \sigma_{i}$ [risp. $\left.s\left(\varrho_{j} \times \sigma_{i}\right)\right]$ rispetto alle $\varphi_{j} \times \psi_{i}(j \in J, i \in I)$. Allora:

a) $\varrho \times \sigma \subset \tau$ e inoltre:

$\left.a^{\prime}\right)$ se $X=\bigcup_{j \in J} \varphi_{j}\left(X_{j}\right), Y=\bigcup_{i \in I} \psi_{i}\left(Y_{i}\right)$ e se per ogni $j \in J, i \in I$ si ha che $\varphi_{j}:\left(X_{j}, \varrho_{j}\right) \rightarrow(X, \varrho)$ e $\psi_{i}:\left(Y_{i}, \sigma_{i}\right) \rightarrow(Y, \sigma)$ sono aperte, risulta che $\varrho \times \sigma=\tau$;

$\left.a^{\prime \prime}\right)$ se per ogni $j \in J$ e $i \in I$ si ha che $\varphi_{i_{X_{j}, \varphi_{j}\left(X_{j}\right)}}:\left(X_{j}, \varrho_{j}\right) \rightarrow\left(\varphi_{j}\left(X_{j}\right), \varrho /_{\varphi_{j}\left(X_{j}\right)}\right)$ e $\psi_{i_{Y_{i}, \psi_{i}\left(Y_{i}\right)}}:\left(Y_{i}, \sigma_{i}\right) \rightarrow\left(\psi_{i}\left(Y_{i}\right), \sigma_{\psi_{i}\left(Y_{i}\right)}\right)$ sono aperte e che $\varphi_{j}^{-1}\left(\varphi_{j}(A)\right)=A$ per ogni $A \in \varrho_{j}$ e $\psi_{i}^{-1}\left(\psi_{i}(B)\right)=B$ per ogni $B \in \sigma_{i}$, se $\left(\varrho,\left\{\varphi_{j}\left(X_{j}\right): j \in J\right\}\right)$ e $\left(\sigma,\left\{\psi_{i}\left(Y_{i}\right): i \in I\right\}\right)$ verificano la proprietà $(L)$, risulta che $\varrho \times \sigma=\tau$;

b) $s\left(\varrho^{s} \times \sigma^{s}\right) \subset \tau^{s}$ e inoltre:

$\left.b^{\prime}\right)$ se $X=\bigcup_{j \in J} \varphi_{j}\left(X_{j}\right), Y=\bigcup_{i \in I} \psi_{i}\left(Y_{i}\right)$ e se per ogni $j \in J, i \in I$ si ha che $\phi_{i} \times \psi_{i}:\left(X_{j} \times Y_{i}, \varrho_{j} \times \sigma_{i}\right) \rightarrow\left(X \times Y, \varrho^{s} \times \sigma^{s}\right)$ è sequenzialmente aperta, risulta che $s\left(Q^{s} \times \sigma^{8}\right)=\tau^{s}$;

$\left.b^{\prime \prime}\right)$ se per ogni $j \in J, i \in I$ si ha che $\varphi_{j_{X_{j}, \varphi_{j}\left(X_{j}\right)}}:\left(X_{j}, \varrho_{j}\right) \rightarrow\left(\varphi_{j}\left(X_{j}\right), \varrho^{8} / \varphi_{j}\left(X_{j}\right)\right)$ e $\psi_{i_{T_{i}, \psi_{i}\left(Y_{i}\right)}}:\left(Y_{i}, \sigma_{i}\right) \rightarrow\left(\psi_{i}\left(Y_{i}\right), \sigma^{s} /_{\psi_{j}\left(Y_{j}\right)}\right)$ sono sequenzialmente aperte e che $\varphi_{j}^{-1}\left(\varphi_{j}(A)\right)=A$ per ogni $A \in s \varrho_{j}$ e $\psi_{i}^{-1}\left(\psi_{i}(B)\right)=B$ per ogni $B \in s \sigma_{i}$, se $\left(\varrho^{s},\left\{\varphi_{j}\left(X_{j}\right): j \in J\right\}\right)$ e $\left(\sigma^{s},\left\{\psi_{i}\left(Y_{i}\right): i \in I\right\}\right)$ verificano la proprietà (sL), risulta che $s\left(\varrho^{s} \times \sigma^{s}\right)=\tau^{s}$.

Dimostrazione. $-a)$ Si noti intanto che se $U \in \underline{Q}, V \in \sigma$ allora $\left(\varphi_{j} \times \psi_{i}\right)^{-1}(U \times V)=$ $=\varphi_{j}^{-1}(U) \times \psi_{i}^{-1}(V) \in \varrho_{j} \times \sigma_{i}$ per ogni $j \in J, i \in I$ e che pertanto la topologia prodotto $\varrho \times \sigma$ è meno fine della topologia $\tau$.

$\left.a^{\prime}\right)$ Sia ora $A \in \tau$. Allora $\left(\varphi_{j} \times \psi_{i}\right)^{-1}(A) \in \varrho_{j} \times \sigma_{i}$ per ogni $j \in J, i \in I$ e per il lemma 1.4 risulta pertanto che $\left(\varphi_{j} \times \psi_{i}\right)\left[\left(\varphi_{j} \times \psi_{i}\right)^{-1}(A)\right] \in \varrho \times \sigma$ per ogni $j \in J, i \in I$, da cui $A=\underset{(j, i) \in J \times I}{\bigcup_{i}}\left(\varphi_{j} \times \psi_{i}\right)\left(\left(\varphi_{j} \times \psi_{i}\right)^{-1}(A)\right) \in \underline{Q} \times \sigma$.

$\left.a^{\prime \prime}\right)$ Basta provare che l'applicazione identica $i_{X \times X}:(X \times Y, \varrho \times \sigma) \rightarrow(X \times Y, \tau)$ è continua. Siano pertanto $(\boldsymbol{K},>)$ insieme diretto, $\left(x_{k}, y_{k}\right),(x, y) \in X \times Y(k \in K)$ tali 
che $\lim _{k \in K}\left(x_{k}, y_{k}\right)=(x, y)$ in $\varrho \times \sigma$. Ora per il teorema 1.18 si ha che $\left(\varrho \times \sigma,\left\{\varphi_{j}\left(X_{j}\right) \times\right.\right.$ $\left.\left.\times \psi_{i}\left(Y_{i}\right): j \in J, i \in I\right\}\right)$ verifica la proprietà $(\mathrm{L})$. Allora per ogni successione generalizzata $S$ estratta da $(k)_{k \in K}$ esistono $(H, \cdot \succ)$ insieme diretto, $(\beta(h))_{h \in H}$ successione generalizzata estratta da $S$ e $j \in J, i \in I$ per cui $x, x_{\beta(h)} \in \varphi_{j}\left(X_{j}\right), y, y_{\beta(h)} \in \psi_{i}\left(Y_{i}\right)$ per ogni $h \in H$. Per $c$ ) del lemma 1.11 si ha ora che, se $z, z_{h} \in X_{j}, t, t_{h} \in Y_{i}$ sono tali che $\varphi_{j}(z)=x, \varphi_{j}\left(z_{h}\right)=x_{\beta(h)}, \psi_{i}(t)=y, \psi_{i}\left(t_{h}\right)=y_{\beta(h)}(h \in H)$, risulta che $\lim _{h \in \mathbb{H}} z_{h}=z$ in $\varrho_{j}$, $\lim _{h \in B} t_{h}=t$ in $\sigma_{i}$ e pertanto, poichè $\varphi_{j} \times \psi_{i}:\left(X_{j} \times Y_{i}, \varrho_{j} \times \sigma_{i}\right) \rightarrow(X \times Y, \tau)$ è continua, si ha che $\lim _{h \in H}\left(x_{\beta(h)}, y_{\beta(h)}\right)=\lim _{h \in H}\left(\varphi_{j}\left(z_{h}\right), \psi_{i}\left(t_{h}\right)\right)=\left(\varphi_{j}(z), \psi_{i}(t)\right)=(x, y)$ in $\tau$. Visto che ciò si può ripetere per ogni successione generalizzata estratta da $(k)_{k \in \mathbb{K}}$, si conclude.

b) Da $e)$ del teorema 1.1 segue intanto che $s\left(\varrho_{j} \times \sigma_{i}\right) \supset s \varrho_{j} \times s \sigma_{i}$ per ogni $j \in J$, $i \in I$. Quindi, se $\tau^{0}$ è la topologia finale delle topologie $s Q_{j} \times 8 \sigma_{i}$ rispetto alle $\varphi_{j} \times \psi_{i}$ $(j \in J, i \in I)$, utilizzando $a)$ si ha che $\varrho^{s} \times \sigma^{s} \subset \tau^{0} \subset \tau^{s}$ e da $\left.a\right)$ del teorema 1.17 e da $\left.e\right)$ del teorema 1.1 si deduce che $s\left(\varrho^{8} \times \sigma^{8}\right) \subset s \tau^{8}=\tau^{8}$.

$\left.b^{\prime}\right)$ Sia $A \in \tau^{s}$. Allora $\left(\varphi_{j} \times \psi_{i}\right)^{-1}(A) \in s\left(\varrho_{j} \times \sigma_{i}\right)$ per ogni $j \in J, i \in I$ e quindi $\left(\varphi_{j} \times \psi_{i}\right)\left[\left(\varphi_{j} \times \psi_{i}\right)^{-1}(A)\right] \in s\left(Q^{s} \times \sigma^{8}\right)$ per ogni $j \in J, i \in I$, da cui $A=\bigcup\left(\varphi_{j} \times \psi_{i}\right)\left(\left(\varphi_{j} \times\right.\right.$ $\left.\left.\times \psi_{i}\right)^{-1}(A)\right) \in s\left(\varrho^{s} \times \sigma^{s}\right)$. $(j, i) \in J \times I$

$\left.b^{\prime \prime}\right)$ se $\tau^{0, s}$ è la topologia finale delle topologie $s\left(s \varrho_{j} \times s \sigma_{i}\right)$ rispetto alle $\varphi_{j} \times \psi_{i}$ $(j \in J, i \in I)$, per $b)$ del teorema 1.1 si ha che

$$
\tau^{s} \subset \tau^{0, s}
$$

e d'altra parte risulta che

$$
\tau^{0, s}=s \tau^{0}
$$

Infatti dalle ipotesi e dal lemma 1.4 segue che $\left(\varphi_{j} \times \psi_{i}\right)_{X_{j} \times Y_{i}, \varphi_{j}\left(X_{j}\right) \times \psi_{i}\left(Y_{i}\right)}:\left(X_{j} \times Y_{i}\right.$, $\left.s \varrho_{j} \times s \sigma_{i}\right) \rightarrow\left(\varphi_{j}\left(X_{j}\right) \times \psi_{i}\left(Y_{i}\right), s\left(\varrho^{s} /_{\varphi_{j}\left(X_{j}\right)}\right) \times s\left(\sigma^{s} /_{\psi_{i}}\left(Y_{i}\right)\right)\right)$ è aperta, d'altra parte si ha che $s\left(\left.\varrho^{s}\right|_{\varphi_{j}\left(X_{j}\right)}\right) \times s\left(\left.\sigma^{s}\right|_{\psi_{i}\left(Y_{i}\right)}\right) \subset s\left(\left.\varrho^{s}\right|_{\varphi_{j}\left(X_{j}\right)} \times \sigma^{s} /_{\psi_{i}\left(Y_{i}\right)}\right)=s\left(\left.\left(\varrho^{s} \times \sigma^{s}\right)\right|_{\varphi_{j}\left(X_{j}\right) \times \psi_{i}\left(Y_{i}\right)}\right) \subset s\left(\left.\tau^{0}\right|_{\varphi_{j}\left(X_{j}\right) \times \psi_{i}\left(Y_{i}\right)}\right)$, ove si è usato $e$ ) del teorema 1.1 per ottenere la prima inclusione, per l'uguaglianza si è tenuto conto del fatto che se $(Z, \zeta)$ e $(W, \theta)$ sono spazi topologici, $E \subset Z, D \subset W$, allora $\left.\zeta\right|_{E} \times\left.\theta\right|_{D}=\left.(\zeta \times \theta)\right|_{E \times D}$ e per l'ultima inclusione si è sfruttato il fatto che per a) si ha che

$$
Q^{8} \times \sigma^{s} \subset \tau^{0}
$$

pertanto

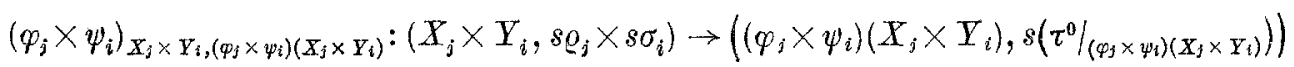

è aperta $(j \in J, i \in I)$; d'altra parte dalle ipotesi e da $k)$ del teorema 1.7 segue che $\left(\varphi_{j} \times \psi_{i}\right)^{-1}\left(\left(\varphi_{j} \times \psi_{i}\right)(E)\right)=E$ per ogni $E \in s \varrho_{j} \times s \sigma_{i}(j \in J, i \in I)$; inoltre dalle ipotesi e dal teorema 1.18 segue che $\left(\varrho^{s} \times \sigma^{s},\left\{\varphi_{j}\left(X_{i}\right) \times \psi_{i}\left(Y_{i}\right): j \in J, i \in I\right\}\right)$ verifica 
la proprietà (sL) e, tenendo conto di (1.21.2), si ha che anche $\left(\tau^{0},\left\{\left(\varphi_{j} \times \psi_{i}\right)\left(X_{j} \times Y_{i}\right)\right.\right.$ : $j \in J, i \in I\})$ la verifica. Pertanto, applicando $c$ ) del teorema 1.19, si ottiene (1.21.1).

Si ha anche che

$$
s \tau^{0} \subset s\left(\varrho^{8} \times \sigma^{s}\right) .
$$

Per provarlo basta dimostrare che $i_{X \times Y}:\left(X \times Y, s\left(\varrho^{s} \times \sigma^{s}\right)\right) \rightarrow\left(X \times Y, s \tau^{0}\right)$ è continua e cioè che $i_{X \times Y}:\left(X \times Y, \varrho^{s} \times \sigma^{s}\right) \rightarrow\left(X \times Y, \tau^{0}\right)$ è sequenzialmente continua e cioè, per $f)$ del teorema 1.1, basta provare che $i_{X \times Y}:\left(X \times Y, \varrho^{s} \times \sigma^{s}\right) \rightarrow\left(X \times Y, \tau^{0}\right)$ verifica $\left.f^{\prime \prime}\right)$. Siano allora $\left(x_{n}, y_{n}\right),(x, y) \in X \times Y(n \in N)$ tali che $\lim _{n \rightarrow \infty}\left(x_{n}, y_{n}\right)=(x, y)$ in $\varrho^{s} \times \sigma^{s}$. Ora per il teorema 1.18 si ha che $\left(\varrho^{s} \times \sigma^{s},\left\{\varphi_{j}\left(X_{j}\right) \times \psi_{i}\left(Y_{i}\right): j \in J, i \in I\right\}\right)$ verifica la proprietà (sL). Allora per ogni sottosuccessione $S$ di $(n)_{n \in N}$ esistono $\left(n_{k}\right)_{k \in N}$ sucessione estratta da $S$ e $j \in J, i \in I$ per cui $x, x_{n_{k}} \in p_{j}\left(X_{j}\right), y, y_{n_{k}} \in \psi_{i}\left(Y_{i}\right)$ per ogni $k \in N$. Per $d$ ) del lemma 1.11 si ha quindi che, se $z, z_{k} \in X_{j}, t, t_{k} \in Y_{i}$ sono tali che $\varphi_{j}(z)=x, \varphi_{j}\left(z_{k}\right)=x_{n_{k}}, \psi_{i}(t)=y, \psi_{i}\left(t_{k}\right)=y_{n_{k}}(k \in N)$, risulta che $\lim _{k \rightarrow \infty} z_{k}=z$ in $s \varrho_{j}$, $\lim _{k \rightarrow \infty} t_{k}=t$ in $s \sigma_{i}$ e pertanto, poichè $\varphi_{j} \times \psi_{i}:\left(X_{j} \times Y_{i}, s \varrho_{j} \times s \sigma_{i}\right) \rightarrow\left(X \times Y, \tau^{0}\right)$ è continua, si ha che $\lim _{k \rightarrow \infty}\left(x_{n_{k}}, y_{n_{k}}\right)=\lim _{k \rightarrow \infty}\left(\varphi_{j}\left(z_{k}\right), \psi_{i}\left(t_{k}\right)\right)=\left(\varphi_{j}(z), \psi_{i}(t)\right)=(x, y)$ in $\tau^{0}$. Visto che ciò si può ripetere per ogni sottosuccessione di $(n)_{n \in N}$, si conclude. Ora dalle relazioni (1.21.0), (1.21.1), (1.21.3) segue che:

$$
\tau^{s} \subset \tau^{0, s}=s \tau^{0} \subset s\left(\varrho^{s} \times \sigma^{s}\right) .
$$

1.22 Esenpio. - Si noti che, nelle condizioni del teorema 1.21, se non valgono le ipotesi di $\left.\left.a^{\prime}\right), a^{\prime \prime}\right)$ [risp. $\left.\left.\left.b^{\prime}\right), b^{\prime \prime}\right)\right]$, può accadere che sia $\varrho \times \sigma \neq \tau$ [risp. $\left.s\left(\varrho^{s} \times \sigma^{s}\right) \neq \tau^{s}\right]$ anche se $Y_{i}=Y$ e $\psi_{i}$ sono le applicazioni identiche $(i \in I)$ e anche se $J=\{0\}$.

Basta considerare $X=\boldsymbol{R}, X_{0}=\{0\}, Y=\boldsymbol{R}, \varrho=\delta, \varrho_{0}=\{\emptyset,\{0\}\}, \sigma=\eta, \varphi_{0}: X_{0} \rightarrow X$, $\varphi(0)=0$. E allora ovvio che $\delta$ è la topologia finale della topologia $\varrho_{0}$ rispetto a $\varphi_{0}$. Allora $\varrho \times \sigma=\delta \times \eta$ e $\tau / \boldsymbol{R}^{2} \backslash\left(\varphi_{0} \times i_{\boldsymbol{R}^{\prime}}\right)(\{0\} \times \boldsymbol{R})=\tau /_{\boldsymbol{R}^{2} \backslash(\{0\} \times \boldsymbol{R})}$ è discreta, mentre

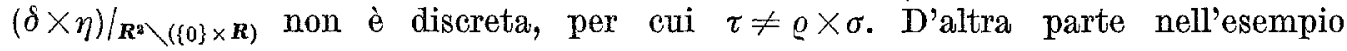
considerato si ha che $\varrho^{8}=\varrho=\delta, \sigma^{s}=\sigma=\eta, s\left(\varrho_{0} \times \sigma\right)=\varrho_{0} \times \eta$, poichè $\varrho_{0}, \eta, \varrho_{0} \times \eta$ sono a base numerabile di intorni e per $b$ ) del teorema 1.1. Allora $\tau^{s}=\tau$ e quindi $\tau^{s} \neq \varrho^{s} \times \sigma^{s}$ e, visto che, ancora per $\left.b\right)$ del teorema 1.1, si ha che $s\left(\varrho^{s} \times \sigma^{s}\right)=s(\delta \times \eta)=$ $=\delta \times \eta=\varrho^{s} \times \sigma^{s}$, risulta anche che $\tau^{s} \neq s\left(\varrho^{s} \times \sigma^{s}\right)$.

1.23 Teorema. - Siano $\left(Z_{k}, \zeta_{k}\right),(Z, \zeta)$ spazi topologici, $\theta_{k}: Z_{k} \rightarrow Z(k \in K), E \subset Z$ e valga almeno uno dei due seguenti gruppi di condizioni:

a) sia $\zeta$ [risp. $s \zeta$ ] la topologia finale delle topologie $\zeta_{k}$ [risp. $s \zeta_{k}$ ] rispetto alle $\theta_{k}$ $(k \in K)$ e valga almeno uno tra:

$\left.a^{\prime}\right) E \in \zeta$ [risp. $\left.E \in s \zeta\right]$,

$\left.a^{\prime \prime}\right) E$ sia chiuso in $\zeta$ [risp. in $s \zeta$ ];

b) $\operatorname{sia} \theta_{k}:\left(Z_{k}, \zeta_{k}\right) \rightarrow(Z, \zeta)$ continua [risp. sequenzialmente continua] per ogni $k \in K$ e valga almeno uno tra: 
$\left.b^{\prime}\right) \theta_{k_{Z_{k}}, \theta_{k}\left(Z_{k}\right)}:\left(Z_{k}, \zeta_{k}\right) \rightarrow\left(\theta_{k}\left(Z_{k}\right),\left.\zeta\right|_{\theta_{k}\left(Z_{k}\right)}\right) \quad\left[\right.$ risp. $\theta_{k_{Z_{k}, \theta_{k}\left(Z_{k}\right)}}:\left(Z_{k}, \zeta_{k}\right) \rightarrow\left(\theta_{k}\left(Z_{k}\right)\right.$, $\left.\delta\left(\left.\zeta\right|_{\theta_{k}\left(Z_{k}\right)}\right)\right]$ siano aperte per ogni $k \in K$, sia $\theta_{k}^{-1}\left(\theta_{k}(A)\right)=A$ per ogni $A \in \zeta_{k}$ e per ogni $k \in K,\left(\zeta,\left\{\theta_{k}\left(Z_{k_{k}}\right): k \in K\right\}\right)$ verifichi la proprietà (L) [risp. (sL)];

$\left.b^{\prime \prime}\right) \theta_{k}:\left(Z_{k}, \zeta_{k}\right) \rightarrow(Z, \zeta)$ [risp. $\theta_{k}:\left(Z_{k}, s \zeta_{k}\right) \rightarrow(Z, s \zeta)$ ] siano aperte per ogni $k_{k} \in K$ e $\operatorname{sia} Z=\bigcup_{k \in K} \theta_{k}\left(Z_{k}\right)$ [risp. sia $Z=\bigcup_{k \in \mathbb{K}} \theta_{k}\left(Z_{k}\right)$ e inoltre sia $\theta_{k}^{-1}\left(\theta_{k}(A)\right)=$ $=A$ per ogni $A \in \zeta_{k}$ e per ogni $k \in K$ ]

Allora $\zeta /_{E}$ [risp. $\left.s\left(\zeta /_{E}\right)\right]$ è la topologia finale delle topologie $\zeta_{k} / \theta_{k}^{-1}(E)$ [risp. $\left.s\left(\zeta_{k} / \theta_{k}^{-1}(E)\right)\right]$ rispetto alle $\theta_{k_{k}^{-1}(E), E}(k \in K)$.

Dmostrazione. - Sia che valga $a)$ e sia che valga $b$ ) si ha che $\theta_{k \theta_{k}^{-1}(E), E}$ : $\left(\theta_{k}^{-1}(E), \zeta_{k} / \theta_{k}^{-1}(E)\right) \rightarrow\left(E, \zeta /_{E}\right)$ sono continue [risp. sequenzialmente continue (utilizzando $f$ ) del teorema 1.1)] per ogni $k \in K$. Sia ora $D \subset E$ tale che $\theta_{k}^{-1}(D) \in \zeta_{k} / \theta_{k}^{-1}(E)$ [risp. $\left.\theta_{k}^{-1}(D) \in s\left(\zeta_{k} / \theta_{k}^{-1}(E)\right)\right]$ per ogni $k \in K$. Per concludere basta allora provare che $\left.D \in \zeta\right|_{E}$ [risp. $\left.D \in s\left(\left.\zeta\right|_{E}\right)\right]$. Se vale $a$ ) con la condizione $a^{\prime}$ ) risulta che $\theta_{k}^{-1}(E) \in \zeta_{k}$ [risp. $\theta_{k}^{-1}(E) \in s \zeta_{k}$ ] e pertanto $\theta_{k}^{-1}(D) \in \zeta_{k}$ [risp. $\theta_{k}^{-1}(D) \in s \zeta_{k}$ (usando $d$ ) del teorema 1.1)] per ogni $k \in K$, per cui $D \in \zeta$ [risp. $D \in s \zeta]$ e quindi $D \in \zeta /_{E}\left[\right.$ risp. $D \in(s \zeta) /_{E} \subset s(\zeta / E)$ (per d) del teorema 1.1)]. Se vale $a)$ con la condizione $a^{\prime \prime}$ ) risulta che $\theta_{k}^{-1}(E)$ è chiuso in $\zeta_{k}$ [risp. in $s \zeta_{k}$ ] e pertanto $\theta_{k}^{-1}(E \backslash D)=\theta_{k}^{-1}(E) \backslash \theta_{k}^{-1}(D)$ è chiuso in $\zeta_{k}$ [risp. in $s \zeta_{k}$ (usando $d$ ) del teorema 1.1)] per ogni $k \in K$, per cui $E \backslash D$ è chiuso in $\zeta$ [risp. in $s \zeta$ ], da cui segue che $E \cap(X \backslash(E \backslash D))=E \cap((X \backslash E) \cup D)=\left.D \in \zeta\right|_{E}$ [risp. $\left.D \in(s \zeta)\right|_{E} \subset$ $c s\left(\left.\zeta\right|_{E}\right)$ (per $d$ ) del teorema 1.1)]. Se ora vale $b$ ) con la condizione $b^{\prime}$ ) e se per assurdo $\left.D \notin \zeta\right|_{E}$ [risp. $D \notin s\left(\left.\zeta\right|_{E}\right)$ ], allora esistono $z \in D,(I,>)$ insieme diretto e $z_{i} \in \mathbb{D} D$ $(i \in I)$ tali che $\lim _{i \in I} z_{i}=z$ in $\zeta$ [risp. esistono $t \in D$ e $t_{n} \in E \backslash D(n \in N)$ tali che $\lim _{n \rightarrow \infty} t_{n}=t$ in $\left.\zeta\right]$ e poichè $\left(\zeta,\left\{\theta_{k}\left(Z_{k}\right): k \in K\right\}\right)$ verifica la proprietà (L) [risp. (sL)] esistono $k \in K,(J, \cdot>)$ insieme diretto e $(\beta(j))_{j \in J}$ successione generalizzata estratta da $(i)_{i \in I}$ tali che $z, z_{\beta(j)} \in \theta_{k}\left(Z_{k}\right)$ per ogni $j \in J$ [risp. esistono $k \in K$ e $\left(n_{h}\right)_{h \in N}$ successione strettamente crescente di naturali tali che $t, t_{n_{h}} \in \theta_{k}\left(Z_{k}\right)$ per ogni $h \in N$ ] e pertanto esistono $w, w_{j} \in Z_{k}(j \in J)$ [risp. $s, s_{h} \in Z_{k}(h \in N)$ ] tali che $\theta_{k}(w)=z, \theta_{k}\left(w_{j}\right)=z_{\beta(j)}$ $(j \in J)$ [risp. $\theta_{k}(s)=t, \theta_{k i}\left(s_{h}\right)=t_{n_{h}}(h \in N)$ ] e per $\left.c\right)$ [risp. $\left.d\right)$ ] del lemma 1.11 risulta che $\lim _{j \in J} w_{j}=w$ [risp. $\left.\lim _{h \rightarrow \infty} s_{k}=s\right]$ in $\zeta_{k}$; d'altra parte $w \in \theta_{k}^{-1}(\{z\}) \subset \theta_{k}^{-1}(D) \in \zeta_{k} / \theta_{k}^{-1}(E)$, $w_{j} \in \theta_{k}^{-1}\left(\left\{z_{\beta(j)}\right\}\right) \subset \theta_{k}^{-1}(E)$ per ogni $j \in J$ [risp. $s \in \theta_{k}^{-1}(\{t\}) \subset \theta_{k}^{-1}(D) \in s\left(\zeta_{k} / \theta_{k}^{-1}(E)\right), s_{h} \in$ $\in \theta_{k}^{-1}\left(\left\{t_{n_{h}^{*}}^{*}\right\}\right) \subset \theta_{k}^{-1}(E)$ per ogni $\left.h \in N\right]$ e pertanto esiste $j^{0} \in J$ [risp. $\left.h^{0} \in N\right]$ tale che $w_{j} \in \theta_{k}^{-1}(D)$ [risp. $\left.s_{h} \in \theta_{k}^{-1}(D)\right]$ per ogni $j \cdot \succ j^{0}$ [risp. $h>h^{0}$ ] e quindi $z_{\beta(j)}=\theta_{k}\left(w_{j}\right) \in$ $\in \theta_{k}\left(\theta_{k}^{-1}(D)\right) \subset D$ per ogni $j \cdot>j^{0}\left[\right.$ risp. $t_{n_{h}}=\theta_{k}\left(s_{h}\right) \in \theta_{k}\left(\theta_{k}^{-1}(D)\right) \subset D$ per ogni $h>h^{0}$ ], il che è assurdo. Valga infine $b$ ) con la condizione $\left.b^{\prime \prime}\right)$. Allora, poichè $\theta_{k}^{-1}(D) \in \zeta_{k} / \theta_{k}^{-1}(B)$, esiste $D_{k} \in \zeta_{k}$ tale che $\theta_{k}^{-1}(D)=D_{k} \cap \theta_{k}^{-1}(E)(k \in K)$ e si ha che $D=\bigcup_{k \in K} \theta_{k}\left(\theta_{k}^{-1}(D)\right)=$ $=\bigcup_{k \in K} \theta_{k}\left(D_{k} \cap \theta_{k}^{-1}(E)\right)=\left(\bigcup_{k \in K} \theta_{k}\left(D_{k}\right)\right) \cap E$, ove l'ultima eguaglianza si prova nel se- 
guente modo:

$$
\begin{aligned}
\bigcup_{k \in \mathbb{K}} \theta_{k}\left(D_{k} \cap \theta_{k}^{-1}(E)\right) \subset \bigcup_{k \in \mathbb{K}}\left[\theta_{k}\left(D_{k}\right) \cap \theta_{k}\left(\theta_{k}^{-1}(E)\right)\right] \subset\left(\bigcup_{k \in K} \theta_{k}\left(D_{k}\right)\right) \cap\left(\bigcup_{k \in \mathbb{K}} \theta_{k}\left(\theta_{k}^{-1}(E)\right)\right) & = \\
= & \left(\bigcup_{k \in \mathbb{K}} \theta_{k}\left(D_{k}\right)\right) \cap E
\end{aligned}
$$

e viceversa se $z \in\left(\bigcup_{k \in K} \theta_{k}\left(D_{k}\right)\right) \cap E$ esiste $k \in K$ tale che $z \in \theta_{k}\left(D_{k}\right) \cap D$, pertanto esiste $z^{0} \in D_{k}$ tale che $\theta_{k}\left(z^{0}\right)=z$ e risulta $z^{0} \in \theta_{k}^{-1}(\{z\}) \subset \theta_{k}^{-1}(E)$, da cui $z=\theta_{k}\left(z^{0}\right) \in \theta_{k}\left(D_{k} \cap\right.$ $\left.\cap \theta_{k}^{-\mathbf{1}}(E)\right)$. Pertanto $\left.D \in \zeta\right|_{k}$, visto che $\bigcup \theta_{k}\left(D_{k}\right) \in \zeta$. [Risp.: sia $k \in K$. Allora si has che

$$
\theta_{k}\left(\theta_{k}^{-1}(D)\right) \in s\left(\left.\zeta\right|_{E \cap \theta_{k}\left(Z_{k}\right)}\right)
$$

Infatti, se per assurdo (1.23.0) non vale, esistono $z_{n}, z \in E \cap \theta_{k}\left(Z_{k}\right), z_{n} \notin \theta_{k}\left(\theta_{k}^{-1}(D)\right)$, $z \in \theta_{k}\left(\theta_{k}^{-1}(D)\right)$, con $\lim _{n \rightarrow \infty} z_{n}=z$ in $\zeta$, allora esistono $w_{n}, w \in \theta_{k}^{-1}(E), w \in \theta_{l}^{-1}(D)$ tali che $\theta_{k}\left(w_{n}\right)=z_{n}, \theta_{k}(w)=z$. Poichè ora $\zeta_{k} \subset s \zeta_{k}$ per $\left.b\right)$ del teorema 1.1, dalle ipotesi segue che se $A \in \zeta_{k}$ allora $\theta_{k}(A) \in s \zeta$ e $\theta_{k}(A) \subset \theta_{k}\left(Z_{k}\right)$, per cui $\left.\theta_{k}(A) \in(s \zeta)\right|_{\theta_{k}\left(Z_{k}\right)} \subset s\left(\left.\zeta\right|_{\theta_{k}\left(Z_{k}\right)}\right)$ (si è utilizzato $d$ ) del teorema 1.1) e pertanto valgono le ipotesi di $d$ ) del lemma 1.11. Quindi $\lim _{n \rightarrow \infty} w_{n}=w$ in $\zeta_{k}$; d'altra parte per ogni $n \in N$ si ha che $w_{n} \notin \theta_{k}^{-1}(D)$, visto che altrimenti $z_{n} \in \theta_{k}\left(\theta_{k}^{-1}(D)\right)$, e $\theta_{k}^{-1}(D) \in s\left(\zeta_{k} / \theta_{k}^{-1}(E)\right)$, per cui $w \notin \theta_{k}^{-1}(D)$, il che è assurdo. Pertanto vale (1.23.0).

D'altra parte dalle ipotesi per ogni $k \in K$ segue che $\theta_{k}\left(Z_{k}\right) \in s \zeta$, da cui $E \cap \theta_{k}\left(Z_{k}\right) \in$ $\left.\in(s \zeta)\right|_{E} \subset s\left(\left.\zeta\right|_{E}\right)$ (si ̀े usato d) del teorema 1.1) e quindi per $d$ ) del teorema 1.1 si ha che

$$
s\left(\left.\zeta\right|_{E \cap \theta_{k}\left(Z_{k}\right)}\right)=s\left(\left.\left(\left.\zeta\right|_{E}\right)\right|_{E \cap \theta_{k}\left(Z_{k}\right)}\right)=\left.\left(s\left(\left.\zeta\right|_{E}\right)\right)\right|_{E \cap \theta_{k}\left(Z_{k}\right)} \subset s\left(\zeta /_{E}\right)
$$

Allora da (1.23.0) segue che $\left.\theta_{k}\left(\theta_{k}^{-1}(D)\right) \in s(\zeta)_{E}\right)$ per ogni $k \in K$, da cui $\left.\left.D=\bigcup_{k \in K} \theta_{k}\left(\theta_{k}^{-1}(D)\right) \in s(\zeta /)_{E}\right) \cdot\right]$

1.24 EsEMPI. - $a)$ Si noti che, se $\left(Z_{k}, \zeta_{k}\right),(Z, \zeta), \theta_{k}(k \in K), E$ sono come nel teorema 1.23, anche se $\zeta$ è la topologia finale delle topologie $\zeta_{k}$ rispetto alle $\theta_{k}(k \in K)$, anche se $E$ d̀ nell'anello generato da $\zeta$ (cfr. [11], $\S 4$ e 5) e $Z_{k}, Z$ sono finiti per cui $\zeta_{k}, \zeta$ sono a base numerabile di aperti e quindi $s \zeta_{k}=\zeta_{k}, s \zeta=\zeta$ per $b$ ) del teorema $1.1(k \in K)$ e anche se $K=\{0\}$ e $\theta_{0}$ è surgettiva (in particolare, quindi, anche se $\left(\zeta,\left\{\theta_{k}\left(Z_{k}\right): k \in K\right\}\right)$ verifica la proprietà $\left.(\mathrm{L})\right)$, ma se non valgono nè $\left.a\right)$ nè $\left.b\right)$ del teorema 1.23 , la tesi del teorema 1.23 può non valere.

Basta considerare $Z_{0}=\{a, b, c, d, e\}, \zeta_{0}=\{\{a, b, d\},\{c, d\},\{c, e\},\{c\},\{d\},\{a, b, c$, $\left.d\},\{c, d, e\}, Z_{0}, \emptyset\right\}, Z=\{f, g, h\}, \theta_{0}(a)=\theta_{0}(b)=f, \theta_{0}(e)=g, \theta_{0}(d)=\theta_{0}(e)=h$. Allora $\zeta=\{\{g\},\{g, h\}, Z, \emptyset\}$ e pertanto, se $E=\{f, g\}$, si ha che $E$ è nell'anello generato da $\zeta$ visto che $\{f\}$ è chiuso in $\zeta$ e $\{g\} \in \zeta$ e d'altra parte risulta che $\{f\} \subset E, \theta_{0}^{-1}(\{f\})=$ 
AdA BotTaro ARUfFo: Su alcune proprietà delle topologie, eece.

$=\{a, b\} \in \zeta_{\mathbf{0}} / \theta_{\mathbf{0}}^{-1}(E) \quad$ poichè $\quad\{a, b\}=\{a, b, d\} \cap\{a, b, c\}=\{a, b, d\} \cap \theta_{0}^{-1}(E)$, mentre $\left.\{f\} \notin \zeta\right|_{E^{*}}$.

b) Si noti che, se vale $b$ ) del teorema 1.23 (nel caso sequenziale) con la condizione $b^{\prime \prime}$ ) eccettuata la sola ipotesi che $\operatorname{sia}_{k}^{-1}\left(\theta_{k}(A)\right)=A$ per ogni $A \in \zeta_{k}$ e per ogni $k_{i} \in K$ e anche se $K=\{0\}$ e $\theta_{0}$ è surgettiva (in particolare, quindi, anche se $\left(\zeta,\left\{\theta_{k}\left(Z_{k}\right)\right.\right.$ : $k \in K\})$ verifica la proprietà $(\mathrm{sL}))$, la tesi del teorema 1.23 può non valere.

Basta considerare $Z=\{a, b, c, d\}, \zeta=\{\{d\},\{b, d\},\{b, c, d\}, Z, \emptyset\}, Z_{0}$ uno spazio di Hilbert separabile e di dimensione infinita su $\boldsymbol{R}, \zeta_{0}=w\left(\boldsymbol{Z}_{0}\right), C=\left\{\boldsymbol{e}_{n}: n \in \boldsymbol{Z}_{+}\right\}$ sistema O.N.C. in $Z_{0}, B=\left\{n e_{k}+e_{n}: n, k \in \boldsymbol{Z}_{+}\right\}, \theta_{0}(0)=a, \theta_{0}(B)=\{b\}, \theta_{0}(C)=\{c\}$, $\theta_{0}\left(Z_{0} \backslash(\{0\} \cup B \cup C)\right)=\{d\}$ (ciò è possibile visto che $\{0\}, B$ e $C$ sono a due a due disgiunti: infatti $\left|n e_{k}+e_{n}\right|_{z_{0}}=\sqrt{n^{2}+1}$ se $n \neq k,\left|n e_{k}+e_{n}\right|_{z_{0}}=n+1$ se $n=k(n$, $\left.k \in \boldsymbol{Z}_{+}\right)$e quindi $|w|_{z_{0}}>1$ per ogni $w \in B$, mentre $|w|_{g_{\mathrm{o}}}=1$ per ogni $\left.w \in C\right)$. Si ha allora che $\theta_{0}:\left(Z_{0}, \operatorname{sw}\left(Z_{0}\right)\right) \rightarrow(Z, s \zeta)=(Z, \zeta)$ è continua e aperta. Infatti $\theta_{0}^{-1}(\{b, c, d\})=$ $=Z_{0} \backslash\{0\} \in \operatorname{sw}\left(Z_{0}\right)$ visto che $\{0\}$ è chiuso in $\operatorname{sw}\left(Z_{0}\right), \theta_{0}^{-1}(\{b, d\})=Z_{0} \backslash(\{0\} \cup O) \in$ $\in \operatorname{sw}\left(Z_{0}\right)$ visto che $\{0\} \cup O$ è chiuso in $\operatorname{sw}\left(Z_{0}\right)$ (se $w_{h}, w \in Z_{0}, w_{h} \in\{0\} \cup O(h \in N)$, $\lim _{h \rightarrow \infty} w_{h}=w$ in $w\left(Z_{0}\right)$ e se $\left(w_{h}\right)_{h \in N}$ non è definitivamente costante si ha che esiste una sottosuccessione di $\left(w_{h}\right)_{h \in N}$ costituita di elementi appartenenti tutti a $C$; allora, se la sottosuccessione considerata non ha estratte costanti, contiene infiniti elementi di $C$ e quindi ha un'estratta in comune con la successione $\left(e_{n}\right)_{n \in Z_{+}}$, per cui $w=0$ ) e $\theta_{0}^{-1}(\{d\})=Z_{0} \backslash(\{0\} \cup B \cup O) \in s w\left(Z_{0}\right)$ visto che $\{0\} \cup B \cup C$ è chiuso in $s w\left(Z_{0}\right)$ (se $w_{h}, w \in Z_{0}, w_{h} \in\{0\} \cup B \cup C(h \in N), \lim _{h \rightarrow \infty} w_{h}=w$ in $w\left(Z_{0}\right)$ si ha che esiste una sottosuccessione di $\left(w_{h}\right)_{h \in N}$ costituita di elementi appartenenti a uno solo dei due insiemi $\{0\} \cup C, B$; se tale insieme $\grave{e}\{0\} \cup C$ allora $w \in\{0\} \cup C$, se ̀̀ $B$ allora per la dimostrazione fatta nell'esempio $1.2 b$ ), se la sottosuccessione considerata non ha un'estratta costante, si ha che $w \in C)$. Pertanto $\theta_{0}:\left(Z_{0}, \operatorname{sq}\left(Z_{0}\right)\right) \rightarrow(Z, \zeta)$ è continua. Per provare ora che è aperta, basta dimostrare che se $A \in s w\left(Z_{0}\right)$ risulta:

(1.24.0) se $0 \in A$ allora $\theta_{0}(A)=Z$;

(1.24.1) se $0 \notin A$ e se $A \cap C \neq \emptyset$ allora $\theta_{0}(A)=\{b, c, d\}$

(1.24.2) se $0 \notin A$, se $A \cap C=\emptyset$ e se $A \cap B \neq \emptyset$ allora $\theta_{0}(A)=\{b, d\}$.

Sia $0 \in A$. Allora, poichè $\lim _{n \rightarrow \infty} e_{n}=0$ in $w\left(Z_{0}\right)$, esiste $n^{0} \in \boldsymbol{Z}_{+}$tale che $e_{n} \in A$ per ogni $n>n^{0}$ e quindi $A \cap C \neq \emptyset$; inoltre, visto che $e_{n^{0}+1} \in A$ e visto che $\lim _{k \rightarrow \infty}\left(\left(n^{0}+1\right) e_{k}+\right.$ $\left.+e_{n^{0}+1}\right)=e_{n^{0}+1}$ in $w\left(Z_{0}\right)$, si ha anche che $A \cap B \neq \emptyset$; infine, visto che $\lim _{n \rightarrow \infty}\left|(1 / n) e_{n}\right|_{z_{0}}=0$, si ha che $\lim _{n \rightarrow \infty}\left((1 / n) e_{n}\right)=0$ in $w\left(Z_{0}\right)$ e quindi $A \cap\left[Z_{0} \backslash(\{0\} \cup B \cup C)\right] \neq \emptyset$, per cui vale $(1.24 .0)$.

Sia $0 \notin A$ e sia $A \cap C \neq \emptyset$; allora, se $n \in Z_{+}$è tale che $e_{n} \in A$, poichè $\lim _{k \rightarrow \infty}\left(n e_{k}+\right.$ $\left.+e_{n}\right)=e_{n}$ in $w\left(Z_{0}\right)$ e poichè $\lim _{k \rightarrow \infty}\left|e_{n}(1-(1 / k))-e_{n}\right|_{z_{0}}=0$, si ha che $A \cap B \neq \emptyset \mathrm{e}$ $A \cap\left[Z_{0} \backslash(\{0\} \cup B \cup C)\right] \neq \emptyset$, per cui vale $(1.24 .1)$. 
Se ora $0 \notin A, A \cap C=\emptyset$ e $A \cap B \neq \emptyset$ allora, se $n, k \in \boldsymbol{Z}_{+}$sono tali che $n e_{k}+$ $+e_{n} \in A$, poichè $\lim _{h \rightarrow \infty}\left|\left(n e_{k}+e_{n}\right)(1+(1 / h))-\left(n e_{k}+e_{n}\right)\right|_{z_{0}}=0$ e poichè $\left(n e_{k}+e_{n}\right)(1+$ $+(1 / h)) \in Z_{0} \backslash(\{0\} \cup B \cup C)$ se $h>1$ visto che $\left\{e_{n}: n \in Z_{+}\right\}$è O.N.C. in $Z_{0}$, si ha che $A \cap\left(Z_{0} \backslash(\{0\} \cup B \cup C)\right) \neq \emptyset$, per cui vale (1.24.2).

Per concludere basta ora considerare $E=\{a, b\}, D=\{a\}$. Si ha infatti che $\theta_{0}^{-1}(D)=\{0\}$ e quindi $\theta_{0}^{-1}(D) \in s\left(w\left(Z_{0}\right) / \theta_{0}^{-1}(E)\right)=s\left(w\left(Z_{0}\right) / B_{B \cup\{0\}}\right)$ (per la dimostrazione fatta nell'esempio 1.20$)$ ), mentre $D \notin s\left(\left.\zeta\right|_{E}\right)$ visto che $\left.\zeta\right|_{E}=\{\{b\},\{a, b\}, \emptyset\}$ e visto che $s\left(\zeta /_{E}\right)=\left.\zeta\right|_{E}$ per $\left.b\right)$ del teorema 1.1 .

1.25 Teorema. - Siano $(Z, \zeta),(W, \theta)$ spazi topologici, $\varphi:(Z, \zeta) \rightarrow(W, \theta)$. Allora, se $\varphi$ è aperta [risp. sequenzialmente aperta], se $E \subset Z, \varphi(E) \subset D \subset W$ e se vale almeno una delle seguenti condizioni:

a) $E \in \zeta$ [risp. $E \in s \zeta]$

b) $\varphi^{-1}(\varphi(E))=E \quad($ o, equivalentemente, $\varphi(Z \backslash E) \cap \varphi(E)=\emptyset), D \backslash \varphi(E) \subset W \backslash$ $\backslash \varphi(Z)\left[\operatorname{risp} . \varphi^{-1}(\varphi(E))=E, D \backslash \varphi(E) \subset W \backslash \varphi(Z), E\right.$ chiuso in $\left.s \zeta\right]$;

o) $\varphi^{-1}(\varphi(A))=A$ per ogni $A \in \zeta, D \backslash \varphi(E) \subset W \backslash \varphi(Z)$

si ha che $\varphi_{E, D}:\left(E,\left.\zeta\right|_{E}\right) \rightarrow(D, \theta / D)$ è aperta [risp. sequenzialmente aperta].

Dimostrazione. - Sia $\varphi$ aperta e sia $\left.A \in \zeta\right|_{E}$. Allora esiste $A^{\prime} \in \zeta$ tale che $A=$ $=E \cap A^{\prime}$ e pertanto, se vale $\left.a\right), A \in \zeta$ e quindi $\phi(A) \in \theta$. Se vale una almeno tra b) e o) si ha che

$$
\varphi(A)=\varphi(E) \cap \varphi\left(\boldsymbol{A}^{\prime}\right)
$$

Infatti se vale $b)$ si ha che $\varphi(A)=\varphi\left(E \cap A^{\prime}\right)$ e, poichè $\varphi\left(A^{\prime}\right)=\varphi\left(A^{\prime} \backslash E\right) \cup$ $\cup \varphi\left(A^{\prime} \cap E\right)$, risulta che $\varphi\left(A^{\prime}\right) \cap \varphi(E)=\left[\varphi\left(A^{\prime} \backslash E\right) \cap \varphi(E)\right] \cup\left[\varphi\left(A^{\prime} \cap E\right) \cap \varphi(E)\right]=$ $=\varphi\left(A^{\prime} \cap E\right)$, ove si è usato che $\varphi(\mathbb{Z} \backslash E) \cap \varphi(E)=\emptyset$; pertanto vale (1.25.0). Valga ora $c)$. Allora $\varphi\left(Z \backslash A^{\prime}\right) \cap \varphi\left(A^{\prime}\right)=\emptyset$. Pertanto, visto che $\varphi(E)=\varphi\left(E \backslash A^{\prime}\right) \cup \varphi\left(E \cap A^{\prime}\right)$, risulta $\varphi(E) \cap \varphi\left(A^{\prime}\right)=\left[\varphi\left(E \backslash A^{\prime}\right) \cap \varphi\left(A^{\prime}\right)\right] \cup\left[\varphi\left(E \cap A^{\prime}\right) \cap \varphi\left(A^{\prime}\right)\right]=\varphi\left(E \cap A^{\prime}\right)$; quindi $\varphi(A)=\varphi\left(E \cap A^{\prime}\right)=\varphi(E) \cap \varphi\left(A^{\prime}\right)$. Ora, utilizzando che $D \backslash \varphi(E) \subset W \backslash \varphi(Z)$, per (1.25.0) risulta che

$$
D \cap \varphi\left(A^{\prime}\right)=\left[(D \backslash \varphi(E)) \cap \varphi\left(A^{\prime}\right)\right] \cup\left[\varphi(E) \cap \varphi\left(A^{\prime}\right)\right]=\varphi(E) \cap \varphi\left(A^{\prime}\right)=\varphi(A)
$$

e pertanto, visto che $\varphi\left(A^{\prime}\right) \in \theta$, si ha che $\varphi(A) \in \theta / D_{D}$.

[Risp.: sia $\varphi$ sequenzialmente aperta. Se vale almeno una tra $a$ ) e $b$ ), allora per $d$ ) del teorema 1.1 risulta che $s\left(\left.\zeta\right|_{E}\right)=\left.(s \zeta)\right|_{E}$. D'altra parte, utilizzando il teorema dimostrato sopra, di cui vale almeno una tra le ipotesi $a$ ) e $b$ ) relativamente alle topologie $s \zeta$ su $Z$ e $s \theta \mathrm{su} \mathrm{W}$, si ottiene che $\varphi_{E, D}:\left(E,(s \zeta) /_{E}\right) \rightarrow\left(D,\left.(s \theta)\right|_{D}\right)$ è aperta e quindi, poichè da $d$ ) del teorema 1.1 segue che $\left.(s \theta)\right|_{D} \subset s(\theta / D)$, si conclude. Valga 
ora $c)$. Sia $A \in s\left(\left.\zeta\right|_{E}\right)$. Allora risulta che

$$
\varphi(A) \in s(\theta / \varphi(H))
$$

Infatti se per assurdo esistessero $w \in \varphi(A), w_{n} \in \varphi(E) \backslash \varphi(A)(n \in N), w_{n} \rightarrow w$ in $\theta$, allora esisterebbero $z \in A, z_{n} \in E \backslash A$ tali che $\varphi(z)=w, \varphi\left(z_{n}\right)=w_{n}(n \in \mathbf{N})$ e pertanto per $d$ ) del lemma 1.11 (che si può usare in quanto per $d$ ) del teorema 1.1 si ha che $\left.\left.(s \theta)\right|_{\varphi(Z)} \subset s\left(\left.\theta\right|_{\varphi(Z)}\right)\right)$ si avrebbe che $z_{n} \rightarrow z$ in $\zeta$, il che è assurdo. Pertanto vale (1.25.1). D'altra parte, visto che $\varphi(Z) \in s \theta$ e che $D \backslash \varphi(E) \subset W \backslash \varphi(Z)$, si ha che $\varphi(E)=$ $=\left.\varphi(Z) \cap D \in(s \theta)\right|_{D} \subset s(\theta / D)$ (si è usato $d$ ) del teorema 1.1) e quindi per $d$ ) del teorema 1.1 risulta che

$$
s(\theta / \varphi(E))=s\left((\theta / D) /_{\varphi(E)}\right)=(s(\theta / D)) /_{\varphi(E)} \subset s(\theta / D)
$$

per cui da (1.25.1) segue che $\varphi(A) \in s(\theta / D) \cdot 1$

1.26 EsEmp. - a) Se non vale nessuna delle condizioni $a), b), c$ ), anche se $E$ è chiuso in $\zeta$ e $D=\varphi(E)$, il teorema 1.25 può non valere.

Basta considerare $Z=\{a, b, c\}, W=\{d, e\}, \zeta=\{\{a\},\{a, b\}, Z, \emptyset\}, \theta=\{\{e\}, W, \emptyset\}$, $\varphi(a)=\varphi(c)=e, \varphi(b)=d$. Allora $\varphi$ è aperta, ma se $E=\{b, c\}$ che è chiuso in $\zeta$, si ha che $\left.\{b\} \in \zeta\right|_{E}$ e $\varphi(\{b\})=\{d\} \notin \theta / \varphi(E)=\theta$.

b) Se, nel caso sequenziale, vale l'ipotesi $b$ ) del teorema 1.25 eccettuata la condizione che $E$ sia chiuso in $s \zeta$, allora la tesi del teorema 1.25 può non valere.

Basta considerare l'esempio $b)$ del n. 1.24. Risulta allora che $\theta_{0}:\left(Z_{0}, s w\left(Z_{0}\right)\right) \rightarrow$ $\rightarrow(Z, s \zeta)$ è aperta. D'altra parte se si considera $E=B \cup\{0\}$ e $D=\theta_{0}(E)=\{a, b\}$, si ha che $\{0\} \in s\left(\left.w\left(Z_{0}\right)\right|_{E}\right)$, mentre $\theta_{0}(\{0\})=\{a\} \notin s\left(\zeta /_{\theta_{0}(E)}\right)$ (come si è visto nell'esempio $1.24 \mathrm{~b})$ ).

1.27 Teorema. - Siano $X_{j}, X$ insiemi, $\varphi_{j}: X_{j} \rightarrow X, \mathfrak{A}_{j} \subset 2^{X_{j}}, \mathfrak{A} \subset 2^{X}(j \in J)$. Allora:

a) se $X=\bigcup_{j \in J} \varphi_{j}\left(X_{j}\right), J \subset N, \varphi_{j}(A) \in \sigma(\mathcal{A})$ per ogni $A \in \sigma\left(\mathcal{A}_{j}\right)$ e per ogni $j \in J$ e se $E \subset X$, risulta che: da $\varphi_{j}^{-1}(E) \in \sigma\left(\mathscr{A}_{j}\right)$ per ogni $j \in J$ segue che $E \in \sigma(\mathcal{A})$.

Siano inoltre $T_{i}, T$ insiemi, $\psi_{i}: T_{i} \rightarrow T, \mathcal{C}_{i} \subset 2^{T_{i}}, \mathcal{C} \subset 2^{T}$ tali che $T_{i} \in \mathcal{C}_{i}(i \in I)$ e $X_{j} \in \mathcal{A}_{j}$ per ogni $j \in J$. Allora:

b) se $X=\bigcup_{j \in J} \varphi_{j}\left(X_{j}\right), J \subset \boldsymbol{N}, \varphi_{j}(A) \in \sigma(A)$ per ogni $A \in \mathfrak{A}_{j}$ e per ogni $j \in J$, $\varphi_{j}^{-1}\left(\varphi_{j}(A)\right)=A$ per ogni $A \in \mathcal{A}_{j}$ e per ogni $j \in J$, se $T=\bigcup_{i \in I} \psi_{i}\left(T_{i}\right), I \subset N$, $\psi_{i}(C) \in \sigma(\mathcal{C})$ per ogni $O \in \mathbb{C}_{i}$ e per ogni $i \in I, \psi_{i}^{-1}\left(\psi_{i}(C)\right)=C$ per ogni $C \in \mathbb{C}_{i} \mathrm{e}$ per ogni $i \in I$ e se $E \subset T \times X$, risulta che:

da $\left(\psi_{i} \times \varphi_{j}\right)^{-1}(\mathbb{E}) \in \sigma\left(\mathcal{C}_{i}\right) \times \sigma\left(\mathcal{A}_{j}\right)$ per ogni $i \in I, j \in J$ segue che $E \in \sigma(\mathcal{C}) \times \sigma(\mathcal{A})$. 
Dimostrazione. - a) Sia $E \subset X$ tale che $\varphi_{j}^{-1}(E) \in \sigma\left(\mathscr{A}_{j}\right)$ per ogni $j \in J$. Allora $E=\bigcup_{j \in J} \varphi_{j}\left(\varphi_{j}^{-1}(E)\right) \in \sigma(\mathfrak{A})$ poichè $\varphi_{j}\left(\varphi_{j}^{-1}(E)\right) \in \sigma(\mathfrak{A})$ per ogni $j \in J$ e poichè $J \subset N$.

b) Sia $E \subset T \times X$ tale che $\left(\psi_{i} \times \varphi_{j}\right)^{-1}(E) \in \sigma\left(\mathcal{C}_{i}\right) \times \sigma\left(\mathcal{A}_{j}\right)$ per ogni $i \in I, j \in J$. Allora $E \underset{(i, j) \in I \times J}{\bigcup_{J}}\left(\psi_{i} \times \varphi_{j}\right)\left[\left(\psi_{i} \times \varphi_{j}\right)^{-1}(E)\right] \in \sigma(\mathcal{C}) \times \sigma(\mathcal{A})$ visto che $\left(\psi_{i} \times \varphi_{j}\right)\left[\left(\psi_{i} \times \varphi_{j}\right)^{-1}(E)\right] \in$ $\in \sigma(\mathcal{C}) \times \sigma(\mathfrak{A})$ per ogni $i \in I, j \in J$ per $c)$ e $j$ ) del lemma 1.7 e visto che $I \subset N$ e $J \subset N$.

1.28 Esemplo. - Siano $\left(X_{j}, \varrho_{j}\right),(X, \varrho)$ spazi topologici, $\varphi_{j}: X_{j} \rightarrow X$ e $\varrho$ sia la topologia finale per le $\varphi_{j}(j \in J)$. Allora la tesi di $a$ ) del teorema 1.27 (relativo ad $\left.\mathcal{A}_{j}=\varrho_{j}, \mathcal{A}=\varrho(j \in J)\right)$ può non valere (anche se $J \subset N$ e $X=\bigcup_{j \in J} p_{j}\left(X_{j}\right)$ ) e cioè possono esistere insiemi $A \subset X, A \notin \mathfrak{B}(\varrho)$ tali che $\varphi_{j}^{-1}(A) \in \mathfrak{B}\left(\varrho_{j}\right)$ per ogni $j \in J$.

Basta considerare $J=\{0,1\}, X_{j}=\left(\boldsymbol{R}, \varrho_{j}\right)(j=0,1), \varrho_{0}=\{j a, \infty[: a \in \boldsymbol{R}\} \cup\{[a$, $\left.\left.\infty[: a \in \boldsymbol{R}\} \cup\{\boldsymbol{R}, \emptyset\}, \varrho_{1}=\{]-\infty, a[: a \in \boldsymbol{R}\} \cup\{]-\infty, a\right]: a \in \boldsymbol{R}\right\} \cup\{\boldsymbol{R}, \emptyset\}, \varphi_{j}=i_{\boldsymbol{R}}(j=$ $=0,1)$. Allora $(X, \varrho)=(\boldsymbol{R}, \varrho)$, ove $\varrho$ è la topologia banale. D'altra parte si ha che $\mathfrak{B}\left(\varrho_{j}\right) \supset \varrho_{0} \cup \varrho_{1}(j=0,1)$ e quindi ogni $A \in \varrho_{0} \cup \varrho_{1}$ è tale che $\left(i_{\boldsymbol{R}}\right)^{-1}(A) \in \mathfrak{B}\left(\varrho_{j}\right)(j=$ $=0,1)$, mentre $\mathcal{B}(\varrho)=\{\boldsymbol{R}, \emptyset\}$.

1.29 Teorema. - Siano $I \subset N, T, T_{i}(i \in I)$ insiemi, $\mathfrak{C}_{i} \sigma$-algebra su $T_{i}(i \in I), \mathcal{L}$ $\sigma$-algebra su $T, \mu_{i}: \mathfrak{L}_{i} \rightarrow[0, \infty](i \in I), \mu: \mathcal{L} \rightarrow[0, \infty]$ misure, $\psi_{i}: T_{i} \rightarrow T, \mathscr{H}_{i} \subset \mathfrak{L}_{i}$, $\pi \subset \mathcal{L}(i \in I)$. Allora:

a) se $\mu$ è $K$-internamente regolare e se vale

(1.29.0) per ogni $i \in I$ esiste $d_{i}>0$ tale che $\psi_{i}(E) \in \mathfrak{L}$ e $\mu\left(\psi_{i}(E)\right) \leqslant d_{i} \mu_{i}(E)$ per ogni $E \in \mathfrak{L}_{i}$

risulta che:

(1.29.1) per ogni $\left(H_{n, i}: n \in \boldsymbol{Z}_{+}\right.$, $\left.i \in I\right)$, con $H_{n, i} \in J_{i}$ tale che $\mu_{i}\left(T_{i} \backslash H_{n, i}\right)<1 / n$ $\left(n \in \boldsymbol{Z}_{+}, i \in I\right)$, esistono $\left(H_{n}: n \in \boldsymbol{Z}_{+}\right)$e $\left(k_{n, i}: n \in \boldsymbol{Z}_{+}, i \in I\right)$ con $H_{n} \in \mathcal{K} \mathrm{e}$ $k_{n, i} \in \boldsymbol{Z}_{+}$tali che $\psi_{i}^{-1}\left(H_{n}\right) \subset H_{k_{n, i}, i}$ e $\mu\left(T \backslash H_{n}\right)<1 / n\left(n \in \boldsymbol{Z}_{+}, i \in I\right)$;

la stessa condizione (1.29.1) si ottiene se vale $(1.29 .0)$, se vale

(1.29.2) $\bigcup_{h=0}^{k} \psi_{i_{h}}\left(K_{h}\right) \in \mathcal{K}$ per ogni $k \in N, i_{h} \in I$ e $K_{h} \in \mathcal{H}_{i_{h}}(h=0, \ldots, k)$; esistono

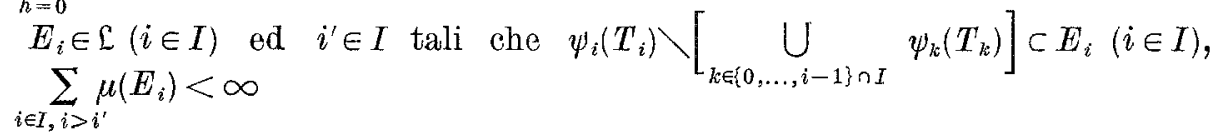

e se vale

$$
K \backslash\left(\bigcup_{i \in I} \psi_{i}\left(T_{i} \backslash K_{i}\right)\right) \in \mathcal{K} \text { per ogni } K_{i} \in \mathcal{H}_{i}(i \in I) \text { e } K \in \mathscr{K}, \mu\left[T \backslash\left(\bigcup_{i \in I} \psi_{i}\left(T_{i}\right)\right)\right]=0
$$


b) se $\mu$ è $\hbar$-internamente regolare e se vale

$$
\begin{aligned}
& \psi_{i}(H) \in \mathcal{L} \text { e } \psi_{i}\left(T_{i}\right) \in \mathcal{L} \text { per ogni } H \in \mathfrak{H}_{i} \text { e } i \in I, \mu\left[T \backslash\left(\bigcup_{i \in I} \psi_{i}\left(T_{i}\right)\right)\right]=0 \text { e per } \\
& \text { ogni } i \in I \text { esiste } d_{i}>0 \text { tale che } \psi_{i}^{-1}(D) \in \mathcal{L}_{i} \text { e } \mu(D) \leqslant d_{i} \mu_{i}\left(\psi_{i}^{-1}(D)\right) \text { per ogni } \\
& D \in \mathfrak{L}, D \subset \psi_{i}\left(T_{i}\right)
\end{aligned}
$$

risulta che:

(1.29.5) per ogni $\left(H_{n, i}: n \in \boldsymbol{Z}_{+}, i \in I\right)$, con $H_{n, i} \in \mathfrak{H}_{i}$ tale che $\mu_{i}\left(T_{i} \backslash H_{n, i}\right)<1 / n(n \in$ $\left.\in \boldsymbol{Z}_{+}, i \in I\right)$, esistono $\left(N_{n}: n \in \boldsymbol{Z}_{+}\right)$, con $N_{n} \subset I\left(n \in \boldsymbol{Z}_{+}\right),\left(H_{n}: n \in \boldsymbol{Z}_{+}\right)$e $\left(k_{n, i}:\right.$ $\left.i \in N_{n}, n \in \boldsymbol{Z}_{+}\right)$con $H_{n} \in \nVdash$ e $k_{n, i} \in \boldsymbol{Z}_{+}\left(i \in N_{n}\right)$ tali che $\mu\left(T \backslash H_{n}\right)<1 / n$, $\boldsymbol{H}_{n}=\bigcup_{i \in \mathbb{N}_{n}} \psi_{i}\left[H_{k_{n, i}, i} \cap \psi_{i}^{-1}\left(\boldsymbol{H}_{n}\right)\right]\left(n \in \boldsymbol{Z}_{+}\right)$;

la stessa condizione (1.29.5) segue dalle ipotesi (1.29.4) e (1.29.2).

Siano inoltre $\tau$ topologia su $T, \tau_{i}$ topologia su $T_{i}(i \in I),(X, \varrho),\left(X_{0}, \varrho_{0}\right)$ spazi topologici, $\varphi: X_{0} \rightarrow X, E \subset T \times X, E_{i}=\left(\psi_{i} \times \varphi\right)^{-1}(E)(i \in I)$. Allora:

c) se vale (1.29.4), se $\varphi$ è aperta [risp. se vale (1.29.4)], se vale (1.29.2) e se vale

(1.29.6) per ogni $i \in I$, per ogni $H \in \mathcal{H}_{i}$, per ogni $\varepsilon>0$ e per ogni $K \in \mathcal{K}$, $K \supset \psi_{i}(H)$ esiste $K_{\varepsilon}^{(i, H, K)} \subset K, K_{\varepsilon}^{(i, H, K)} \in \mathcal{L}$ tale che sia $\mu\left(K \backslash K_{\varepsilon}^{(i, H, K)}\right)<\varepsilon$, $\psi_{i_{H \cap \varphi_{i}^{-1}}\left(K_{\varepsilon}^{(i, H, K)}\right), K_{\varepsilon}^{(i, H, K)}}$ sia aperta [risp. $\left(\psi_{i} \times \varphi\right)_{E_{i} \cap\left[\left(H \cap \psi_{i}^{-1}(L)\right) \times X_{0}\right], E \cap(L \times X)}$ sia sequenzialmente aperta per ogni $\left.L \in \mathcal{K}, L \subset K_{\varepsilon}^{(i, H, K)}\right]$ e inoltre sia $\bigcap_{h=0}^{k} K_{\varepsilon_{h}}^{\left(i_{h}, H_{h}, K_{h}\right)} \in \mathcal{K}$ per ogni $k \in N, i_{h} \in I, H_{h} \in \mathcal{J}_{i_{h}}, K_{h} \in \nVdash, K_{h} \supset \psi_{i_{h}}\left(H_{h}\right)$, $\varepsilon_{h}>0(h=0, \ldots, k)$

risulta che

(1.29.7) per ogni $\left(H_{n, i}: n \in \boldsymbol{Z}_{+}, i \in I\right)$, con $H_{n, i} \in \mathcal{H}_{i}$ tale che $\mu_{i}\left(T_{i} \backslash H_{n, i}\right)<1 / n$ $\left(n \in \boldsymbol{Z}_{+}, i \in I\right)$, esistono $\left(N_{n}: n \in \boldsymbol{Z}_{+}\right)$, con $N_{n} \subset I\left(n \in \boldsymbol{Z}_{+}\right),\left(H_{n}: n \in \boldsymbol{Z}_{+}\right)$e $\left(k_{n, i}: i \in N_{n}, n \in \boldsymbol{Z}_{+}\right) \quad$ con $H_{n} \in \mathcal{K}$ e $k_{n, i} \in \boldsymbol{Z}_{+}\left(i \in N_{n}\right)$ tali che $\mu\left(T \backslash H_{n}\right)<$ $<1 / n, H_{n}=\bigcup_{i \in N_{n}} \psi_{i}\left(H_{k_{n, i}, i} \cap \psi_{i}^{-1}\left(H_{n}\right)\right)$ e

$$
\left(\psi_{i} \times \varphi\right)_{E_{i} \cap\left[\left(H_{k_{n, i}, i} \cap \varphi_{i}^{-1}\left(H_{n}\right)\right) \times X_{0}\right], E \cap\left(H_{n} \times X\right)}
$$

sia aperta [risp.

$$
\left(\psi_{i} \times \varphi\right)_{\left.E_{i} \cap\left[\left(H_{k_{n}, i}, i \cap \psi_{i}^{-1}(L)\right) \times X_{0}\right], E \cap \cap L \times X\right)}
$$

sia sequenzialmente aperta per ogni $\left.L \in \mathscr{K}, L \subset H_{n}\right]$ per ogni $i \in N_{n}, n \in \boldsymbol{Z}_{+}$. 
Dimostrazione. - a) Siano $H_{n, i} \in \mathcal{J}_{i}$ tali che $\mu_{i}\left(T_{i} \backslash H_{n, i}\right)<1 / n\left(i \in I, n \in Z_{+}\right)$. Sia $\mu$ misura $\pi$-internamente regolare e valga $(1.29 .0)$; sia $n \in \boldsymbol{Z}_{+}$e siano $k_{n, i} \in \boldsymbol{Z}_{+}$ $(i \in I)$ tali che $\sum_{i \in I} d_{i} / k_{n, i}<1 /(2 n)$ (ad esempio $\left.k_{n, i}=\left[2^{i+2} n d_{i}\right]+1(i \in I)\right)$ e $K_{n}=T \backslash$ $\backslash\left[\bigcup_{i \in I} \psi_{i}\left(T_{i} \backslash H_{k_{n, i}, i}\right)\right], H_{n} \in \pi, H_{n} \subset K_{n}$ tale che $\mu\left(K_{n} \backslash H_{n}\right)<1 /(2 n)$. Allora $\psi_{i}^{-1}\left(H_{n}\right) \subset$ $\subset \psi_{i}^{-1}\left(K_{n}\right) \subset H_{k_{n, i}, i}$ per ogni $i \in I \quad$ e $\mu\left(T \backslash K_{n}\right)=\mu\left[\bigcup_{i \in I} \psi_{i}\left(T_{i} \backslash H_{k_{n, i}, i}\right)\right] \leqslant \sum_{i \in I} \mu\left(\psi_{i}\left(T_{i} \backslash\right.\right.$ $\left.\left.\backslash H_{k_{n, i}, i}\right)\right) \leqslant \sum_{i \in I} d_{i} \mu_{i}\left(T_{i} \backslash H_{k_{n, i} i}\right) \leqslant \sum_{i \in I} d_{i}\left(1 / k_{n, i}\right)<1 /(2 n)$, da cui $\mu\left(T \backslash H_{n}\right)<1 / n$. Valgano ora $(1.29 .0),(1.29 .2)$ e $(1.29 .3)$ e sia $n \in Z_{+}$. Se $E_{i}(i \in I)$ ed $i^{\prime} \in I$ sono come in (1.29.2), poichè $\sum_{i \in I, i>i^{\prime}} \mu\left(E_{i}\right)<\infty$, esiste $i_{n} \in I, i_{n} \geqslant i^{\prime}$ tale che $\sum_{i \in I, i>i_{n}} \mu\left(E_{i}\right)<1 /(2 n)$. Siano $k_{n, i} \in Z_{+}(i \in I)$ tali che $\sum_{i \in I}\left(d_{i} / k_{n, i}\right)<1 /(2 n) \quad\left(\right.$ ad esempio $\left.k_{n, i}=\left[2^{i+2} n d_{i}\right]+1(i \in I)\right)$ e $H_{n}=\left[\bigcup_{i \in\left\{0, \ldots, i_{n}\right\} \cap I} \psi_{i}\left(H_{k_{n, i}, i}\right)\right] \backslash\left[\bigcup_{i \in I} \psi_{i}\left(T_{i} \backslash H_{k_{n, i}, i}\right)\right]$. Allora $H_{n} \in \mathcal{K}$ per (1.29.3) e poichè $\bigcup_{i_{i}, \cap I} \psi_{i}\left(H_{k_{n, i}, i}\right) \in \nVdash$ per $(1.29 .2)$. Risulta ora che $\psi_{i}^{-1}\left(H_{n}\right) \subset H_{k_{n, i}, i}$ per ogni $i \in I$ e $i \in\left\{0, \ldots, i_{n}\right\} \cap I$

$$
\begin{aligned}
& T \backslash H_{n}=\left(T \backslash\left[\bigcup_{i \in\left\{0, \ldots, i_{n}\right\} \cap I} \psi_{i}\left(H_{k_{n, i}, i}\right)\right]\right) \cup\left[\bigcup_{i \in I} \psi_{i}\left(T_{i} \backslash H_{k_{n, i}, i}\right)\right] \subset\left[T\left(\bigcup_{i \in I} \psi_{i}\left(T_{i}\right)\right)\right] \cup \\
& \cup\left[\left(\bigcup_{i \in I, i>i_{n}} \psi_{i}\left(T_{i}\right)\right) \backslash\left(\bigcup_{i \in\left\{0, \ldots, i_{n}\right\} \cap I} \psi_{i}\left(T_{i}\right)\right)\right] \cup\left[\bigcup_{i \in I} \psi_{i}\left(T_{i} \backslash H_{k_{n, i}, i}\right)\right],
\end{aligned}
$$

per cui

$$
\begin{array}{r}
\mu\left(T \backslash H_{n}\right) \leqslant \mu\left[T \backslash\left(\bigcup_{i \in I} \psi_{i}\left(T_{i}\right)\right)\right]+\mu\left[\left(\bigcup_{i \in I, i>i_{n}} \psi_{i}\left(T_{i}\right)\right) \backslash\left(\bigcup_{i \in\left\{0, \ldots, i_{n}\right\} \cap I} \psi_{i}\left(T_{i}\right)\right)\right]+ \\
+\mu\left[\bigcup_{i \in I} \psi_{i}\left(T_{i} \backslash H_{\bar{k}_{n, i}, i}\right)\right] \leqslant \mu\left(\bigcup_{i \in I, i>i_{n}}\left[\psi_{i}\left(T_{i}\right) \backslash\left(\bigcup_{k \in\{0, \ldots, i-1\} \cap I}^{\bigcup} \psi_{k}\left(T_{k}\right)\right)\right]\right)+ \\
+\sum_{i \in I} d_{i}\left(1 / k_{n, i}\right)<\mu\left(\bigcup_{i \in I, i>i_{n}} E_{i}\right)+(1 /(2 n))<1 / n
\end{array}
$$

(si è tenuto conto del fatto che se $x \in\left(\bigcup_{i \in I, i>i_{n}} \psi_{i}\left(T_{i}\right)\right) \backslash\left(\bigcup_{i \in\left\{0, \ldots, i_{n}\right\} \cap I} \psi_{i}\left(T_{i}\right)\right)$ e se $i_{x}=\min \{i \in$ $\left.\in I: x \in \psi_{i}\left(T_{i}\right)\right\}$ allora $\left.x \in \psi_{i_{x}}\left(T_{i_{x}}\right) \backslash\left(\bigcup_{k \in\left\{0, \ldots, i_{x}-1\right\} \cap I}^{i \in I, i>i_{n}} \psi_{k}\left(T_{k}\right)\right)\right)^{i \in}$.

b) Siano $H_{n, i} \in \mathcal{H}_{i}$ tali che $\mu_{i}\left(T_{i} \backslash H_{n, i}\right)<1 / n\left(i \in I, n \in Z_{+}\right)$. Sia $\mu$ misura Kinternamente regolare e valga (1.29.4); sia $n \in \boldsymbol{Z}_{+}$, siano $k_{n, i} \in \boldsymbol{Z}_{+}(i \in I)$ tali che $\sum_{i \in I}\left(d_{i} / k_{n, i}\right)<1 /(2 n)$ (ad esempio $\left.k_{n, i}=\left[2^{i+2} n d_{i}\right]+1(i \in I)\right)$ e $K_{n}=\bigcup_{i \in I} \psi_{i}\left(H_{k_{n, i}, i}\right), H_{n} \in K$, $H_{n} \subset K_{n}$ tale che $\mu\left(K_{n} \backslash H_{n}\right)<1 /(2 n)$. Allora $H_{n}=\bigcup_{i \in I} \psi_{i}\left(H_{k_{n, i}, i} \cap \psi_{i}^{-1}\left(H_{n}\right)\right)$ : infatti l'inclusione del secondo membro nel primo è ovvia e se $x \in H_{n}$ allora $x \in K_{n}$ e pertanto esistono $i_{x} \in I$ e $y_{x} \in H_{k_{n, t_{x}}, i_{x}}$ tali che $\psi_{i_{x}}\left(y_{x}\right)=x$ e quindi $y_{x} \in\left(\psi_{i_{x}}\right)^{-1}(\{x\}) \subset$ $\left(\psi_{i_{x}}\right)^{-1}\left(H_{n}\right)$. Inoltre risulta che $T \backslash K_{n}=T \backslash\left[\bigcup_{i \in I} \psi_{i}\left(H_{k_{n, i}, i}\right)\right] \subset\left[T \backslash\left(\bigcup_{i \in I} \psi_{i}\left(T_{i}\right)\right)\right] \cup$ 
$\cup\left[\bigcup_{i \in I}\left(\psi_{i}\left(T_{i}\right) \backslash \psi_{i}\left(H_{k_{n, i}, i}\right)\right]\right.$ e pertanto, tenendo conto di (1.29.4), si ha che

$$
\begin{aligned}
\mu\left(T \backslash K_{n}\right) & \leqslant \mu\left[\bigcup_{i \in I}\left(\psi_{i}\left(T_{i}\right) \backslash \psi_{i}\left(H_{\pi_{n, i}, i}\right)\right)\right] \leqslant \sum_{i \in I} \mu\left(\psi_{i}\left(T_{i}\right) \backslash \psi_{i}\left(H_{k_{n, i}, i}\right)\right) \leqslant \\
& \leqslant \sum_{i \in I} d_{i} \mu_{i}\left[\psi_{i}^{-1}\left(\psi_{i}\left(T_{i}\right) \backslash \psi_{i}\left(H_{k_{n, i}, i}\right)\right)\right] \leqslant \sum_{i \in I} d_{i} \mu_{i}\left(T_{i} \backslash H_{k_{n, i}, i}\right) \leqslant \sum_{i \in I}\left(d_{i} / k_{n, i}\right)<1 /(2 n),
\end{aligned}
$$

da cui $\mu\left(T \backslash H_{n}\right)<1 / n$. Valgano ora $(1.29 .4)$ e (1.29.2) e sia $n \in Z_{+}$. Se $E_{i}(i \in I)$ ed $i^{\prime} \in I$ sono come in (1.29.2), poichè $\sum_{i \in I, i>i^{\prime}} \mu\left(E_{i}\right)<\infty$, esiste $i_{n} \in I, i_{n} \geqslant i^{\prime}$ tale che $\sum_{i \in I, i>i_{n}} \mu\left(E_{i}\right)<1 /(2 n)$. Siano $k_{n, i} \in Z_{+}(i \in I)$ tali che $\sum_{i \in I}\left(d_{i} / k_{n, i}\right)<1 /(2 n)$ (ad esempio $\left.k_{n, i}=\left[2^{i+2} n d_{i}\right]+1(i \in I)\right)$ e $H_{n}=\bigcup_{i \in\left\{0, \ldots, i_{n}\right\} \cap I} \psi_{i}\left(H_{k_{n, i}, i}\right)$. Allora $H_{n} \in \mathcal{K}$ per (1.29.2). Ri-
sulta inoltre che

$$
H_{n}=\bigcup_{i \in\left\{0, \ldots, i_{n}\right\} \cap I} \psi_{i}\left(H_{k_{n, i}, i} \cap \psi_{i}^{-1}\left(H_{n}\right)\right)
$$

e

$$
\begin{aligned}
T \backslash H_{n} \subset\left[T \backslash\left(\bigcup_{i \in I} \psi_{i}\left(T_{i}\right)\right)\right] \cup\left(\bigcup_{i \in I, i>i_{n}}\left[\psi_{i}\left(T_{i}\right) \backslash\left(\bigcup_{k \in\{0, \ldots, i-1\} \cap I}^{\cup} \psi_{k}\left(T_{k}\right)\right)\right]\right) \cup \\
\cup\left[\bigcup_{i \in\left\{0, \ldots, i_{n}\right\} \cap I}\left(\psi_{i}\left(T_{i} \backslash \backslash \psi_{i}\left(H_{k_{n,}, i}\right)\right)\right],\right.
\end{aligned}
$$

da cui segue che

$$
\begin{aligned}
\mu\left(I \backslash H_{n}\right) \leqslant \mu\left(\bigcup_{i \in I, i>i_{n}} E_{i}\right)+\mu\left[\bigcup_{i \in\left\{0, \ldots, i_{n}\right\} \cap I}\left(\psi_{i}\left(T_{i}\right) \backslash \psi_{i}\left(H_{k_{n, i}, i}\right)\right)\right]<(1 /(2 n))+ \\
+\sum_{i \in\left\{0, \ldots, i_{n}\right\} \cap I} \mu\left(\psi_{i}\left(T_{i}\right) \backslash \psi_{i}\left(H_{k_{n, i}, i}\right)\right) \leqslant(1 /(2 n))+\sum_{i \in\left\{0, \ldots, i_{n}\right\} \cap I} d_{i} \mu_{i}\left(T_{i} \backslash H_{k_{n, s}, i}\right) \leqslant(1 /(2 n))+ \\
+\sum_{i \in\left\{0, \ldots, i_{n}\right\} \cap I}\left(d_{i} / k_{n, i}\right)<1 / n
\end{aligned}
$$

e quindi si ha la tesi con $N_{n}=\left\{0, \ldots, i_{n}\right\} \cap I\left(n \in \boldsymbol{Z}_{+}\right)$.

c) Siano $H_{n, i} \in \mathscr{H}_{i}$ tali che $\mu_{i}\left(T_{i} \backslash H_{n, i}\right)<1 / n\left(n \in \boldsymbol{Z}_{+}, i \in I\right)$. Sia $n \in \boldsymbol{Z}_{+}$. Poichè valgono (1.29.2) e (1.29.4), se $k_{n, i}, i_{n}(i \in I)$ sono come nella seconda parte della dimostrazione di $b), K_{n}=\bigcup_{i \in\left\{0, \ldots, i_{n}\right\} \cap I} \psi_{i}\left(H_{k_{n, i}, i}\right)$, si ha che $\mu\left(T \backslash K_{n}\right)<1 / n$ e

$$
K_{n}=\bigcup_{i \in\left\{0, \ldots, i_{n}\right\} \cap I} \psi_{i}\left(H_{k_{n, i}, i} \cap \psi_{i}^{-1}\left(K_{n}\right)\right) .
$$

Sia ora $K_{\varepsilon_{n, i}}^{0}$ relativo a $i, H_{k_{2 n, i}, i}, K_{2 n}$ ed a $\varepsilon_{n, i}=1 /\left(2^{i+2} n\right)\left(i \in\left\{0, \ldots, i_{2 n}\right\} \cap I\right)$ come in (1.29.6). Se ora si considera $H_{n}=\bigcap_{i \in\left\{0, \ldots, i_{2} n\right\} \cap I} K_{\varepsilon_{n, i}}^{0}$, risulta che

$$
\begin{aligned}
H_{n} \in \mathcal{K}, \mu\left(T \backslash H_{n}\right) \leqslant \mu( & \left.\backslash K_{2 n}\right)+\mu\left(K_{2 n} \backslash H_{n}\right)<(1 /(2 n))+ \\
& +\sum_{i \in\left\{0, \ldots, i_{2} n\right\} \cap I} \mu\left(K_{2 n} \backslash K_{\varepsilon_{n, i}}^{0}\right)<(1 /(2 n))+\sum_{i \in\left\{0, \ldots, i_{2 n}\right\} \cap I}\left(1 /\left(2^{i+2} n\right)\right) \leqslant 1 / n,
\end{aligned}
$$


da (1.29.8) segue che $H_{n}=\bigcup_{i \in\left\{0, \ldots, i_{2} n\right\} \cap I} \psi_{i}\left(H_{k_{2} n, i} \cap \psi_{i}^{-1}\left(H_{n}\right)\right)$. Infine da (1.29.6) segue

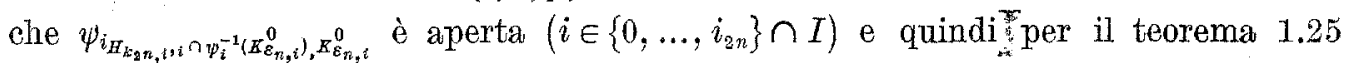
con l'ipotesi $b$ ) risulta che $\psi_{i_{H_{k n}, i, i} \cap \psi_{i}^{-1}\left(\mathbb{H}_{n}\right), \mathbb{Z}_{n}}$ è aperta $\left(i \in\left\{0, \ldots, i_{2 n}\right\} \cap I\right)$ e, visto che $\varphi$ è aperta per ipotesi, per il lemma 1.4 tale è anche $\left(\psi_{i} \times \varphi\right)_{\left(H_{k_{2} n, i ; i} \cap \psi_{i}{ }^{-1}\left(\boldsymbol{H}_{n}\right)\right) \times X_{0}, \boldsymbol{H}_{n} \times X}$ $\left(i \in\left\{0, \ldots, i_{2 n}\right\} \cap I\right)$ e quindi tenendo conto di nuovo del teorema 1.25 con l'ipotesi $\left.b\right)$ si ottiene $(1.29 .7)$ con $N_{n}=\left\{0, \ldots, i_{2 n}\right\} \cap I\left(n \in \boldsymbol{Z}_{+}\right)$[risp. da (1.29.6) segue che $\left(\psi_{i} \times \varphi\right)_{\left.E_{i} \cap\left[H_{k_{2 n}, i, i} \cap \psi_{i}^{-1}(L)\right) \times X_{0}\right], E \cap(L \times X)}$ è sequenzialmente aperta $\left(i \in\left\{0, \ldots, i_{2 n}\right\} \cap I\right)$ per ogni $L \in \widetilde{K}, L \subset H_{n}$ e quindi si ottiene (1.29.7) con $\left.N_{n}=\left\{0, \ldots, i_{2 n}\right\} \cap I\left(n \in \boldsymbol{Z}_{+}\right)\right]$.

\section{2. - Sulle funzioni $(\mathcal{L} \times \mathfrak{B}(\varrho))$-misurabili e s.c.i.}

\subsection{LeMMa. - Se}

A) $T, T_{i}$ sono insiemi, $(X, \varrho),\left(X_{j}, \varrho_{j}\right)$ sono spazi topologici $(i \in I, j \in J), \psi_{i}: T_{i} \rightarrow T$, $\varphi_{j}: X_{j} \rightarrow X(i \in I, j \in J), E \subset T \times X, E_{i, j}=\left(\psi_{i} \times \varphi_{j}\right)^{-1}(E)$ per ogni $i \in I, j \in J ; f: E \rightarrow$ $\rightarrow[-\infty, \infty], f_{i, j}: E_{i, j} \rightarrow[-\infty, \infty]$ sono tali che $f_{i, j}=f \circ\left[\left.\left(\psi_{i} \times \varphi_{j}\right)\right|_{E_{i, j}}\right]$ per ogni $i \in I, j \in J, T^{0}=\left\{t \in T: x \in E_{t} \mapsto f(t, x) \in[-\infty, \infty]\right.$ è s.c.i. [risp. s.s.c.i. $\left.]\right\}$ e $T_{i, j}^{0}=$ $=\left\{s \in T_{i}: x \in\left(E_{i, j}\right)_{s} \mapsto f_{i, j}(s, x) \in[-\infty, \infty]\right.$ è s.c.i. [risp. s.s.c.i.] $\} \quad(i \in I, j \in J)$

e se

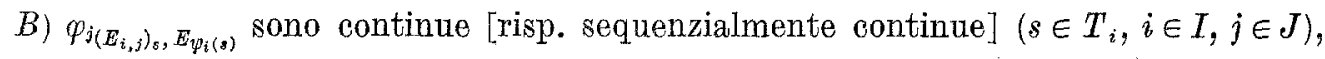

risulta che

$$
\psi_{i}^{-1}\left(T \backslash T^{0}\right) \supset T_{i} \backslash \bigcap_{j \in J} T_{i, j}^{0} \quad \text { per ogni } i \in I
$$

e, se inoltre $N_{i} \subset T_{i}(i \in I)$ sono tali che valga

(2.0.0) per ogni $i \in I$ e per ogni $s \in T_{i} \backslash N_{i}$ si ha che $\varrho /_{E_{v i}(s)}\left[\right.$ risp. $\left.s\left(\varrho / /_{w_{i}(s)}\right)\right]$ è la topologia finale delle topologie $\varrho_{j} /\left(E_{i, j}\right)_{s}\left[\right.$ risp. $\left.s\left(\varrho_{j} /_{\left(E_{i},\right)_{\varepsilon}}\right)\right]$ rispetto alle $\varphi_{j_{\left(E_{i, j}\right)}, E_{\psi_{i}(s)}}(j \in J)$,

si ottiene che

$$
\psi_{i}^{-1}\left(T \backslash T^{\mathbf{0}}\right) \subset\left(T_{i} \backslash \bigcap_{j \in J} T_{i, j}^{0}\right) \cup N_{i} \quad \text { per ogni } i \in I
$$

Dtmostraztone. - Sia $i \in I$. Se $s \in T_{i} \backslash \bigcap_{j \in J} T_{i, j}^{0}$, esiste $j^{0} \in J$ tale che $s \notin T_{i, j^{\circ}}^{0}$ e quindi $f_{i, j^{0}}(s, \cdot)$ non è s.c.i. [risp. non è s.s.c.i.] su $\left(E_{i, j^{0}}\right)_{s} ;$ perciò $f\left(\psi_{i}(s), \cdot\right)$ non è s.c.í. [risp. non è s.s.c.i.] su $E_{\psi_{i}(s)}$, perchè altrimenti $f_{i, j^{0}}(s, \cdot)$ sarebbe s.c.i. [risp. s.s.c.i.] 
su $\left(E_{i, j^{j}}\right)_{\varepsilon}\left(f_{i, j^{0}}(s, y)=f\left(\psi_{i}(s), \varphi_{j^{\circ}}(y)\right)\right.$ per ogni $y \in\left(E_{i, j^{\circ}}\right)_{s}$ e $\varphi_{j^{\circ}\left(E_{i, j^{0}}\right)_{s}, E_{\psi_{i}(s)}}$ è continua per $\left.B\right)$ [risp. sequenzialmente continua per $B$ ) e tenendo conto di $f$ ) e $g$ ) del teorema 1.1]); quindi $\psi_{i}(s) \in T \backslash T^{0}$.

Ora siano $N_{i} \subset T_{i}(i \in I)$ tali che valga $(2.0 .0)$. Sia $i \in I$. Si ha intanto che

$$
\psi_{i}\left[\left(\bigcap_{j \in J} T_{i, j}^{0}\right) \cap\left(T_{i} \backslash N_{i}\right)\right] \subset T^{0} .
$$

Infatti se $t=\psi_{i}(s)$ con $s \in\left(\bigcap_{i \in J} T_{i, j}^{0}\right) \cap\left(T_{i} \backslash N_{i}\right)$ e se $a \in[-\infty, \infty]$, tenendo conto del fatto che $\varphi_{j}^{-1}\left(E_{t}\right)=\left(E_{i, j}\right)_{s}$, si ha che $\left.\left.\left.\left.\varphi_{j}^{-1}\left[(f(t, \cdot))^{-1}(] a, \infty\right]\right)\right]=\left(f_{i, j}(s, \cdot)\right)^{-1}(] a, \infty\right]\right)$, che è in $\varrho_{j} /_{\left(E_{i, j}\right)_{s}}\left[\right.$ risp. in $s\left(\varrho_{j} /\left(E_{i,\rangle_{s}}\right)\right]$ per ogni $j \in J$, per cui da $(2.0 .0)$ segue che $\left.\left.(f(t, \cdot))^{-1}(] a, \infty\right]\right)$ è in $\varrho /_{E_{t}}\left[\right.$ risp. in $\left.s\left(\varrho / E_{E_{t}}\right)\right]$ e quindi $t \in T^{0}$.

Pertanto $\psi_{i}^{-1}\left(T \backslash T^{0}\right) \subset T_{i} \backslash\left[\left(\bigcap_{j \in J} T_{i, j}^{0}\right) \cap\left(T_{i} \backslash N_{i}\right)\right]=\left(T_{i} \backslash \bigcap_{j \in J} T_{i, j}^{0}\right) \cup N_{i}$.

2.1 Teorema. - Valga la seguente condizione:

A) Siano $T_{i}, T$ insiemi, $\mathfrak{L}_{i} \sigma$-algebra su $T_{i}(i \in I), \mathfrak{L} \sigma$-algebra su $T, \mu_{i}: \mathfrak{L}_{i} \rightarrow$ $\rightarrow[0, \infty](i \in I), \mu: \mathcal{L} \rightarrow[0, \infty]$ misure, $(X, \varrho),\left(X_{j}, \varrho_{j}\right), \psi_{i}, \varphi_{j}, E, E_{i, j}, f, f_{i, j}$, $T^{0}, T_{i, j}^{0}(i \in I, j \in J)$ come in $A$ ) del lemma 2.0 .

Allora, se

(2.1.0) $\varphi_{i_{\left(E_{i, j}\right)_{s}, E_{\psi_{i}(s)}}}$ sono continue [risp. sequenzialmente continue] $\left(s \in T_{i}, i \in I, j \in J\right)$

valgono i seguenti fatti:

a) se $\mu_{i}$ sono complete $(i \in I)$ e se

(2.1.1) $A \in \mathfrak{L}, \mu(A)=0$ implica $\psi_{i}^{-1}(A) \in \mathcal{L}_{i}$ e $\mu_{i}\left(\psi_{i}^{-1}(A)\right)=0$ per ogni $i \in I$,

da

(2.1.2) $\quad T^{0} \in \mathcal{E}, \mu\left(T \backslash T^{0}\right)=0$

segue

$T_{i, j}^{0} \in \mathfrak{L}_{i}, \mu_{i}\left(T_{i} \backslash T_{i, j}^{0}\right)=0$ per ogni $i \in I, j \in J$

b) se $J \subset N$ e se

(2.1.4) $\psi_{i}^{-1}(A) \in \mathcal{L}_{i}, \mu_{i}\left(\psi_{i}^{-1}(A)\right)=0$ per ogni $i \in I$ implica $A \in \mathcal{L}$ e $\mu(A)=0$,

(2.1.5) per ogni $i \in I$ esiste $N_{i} \in \mathcal{L}_{i}$ tale che $\mu_{i}\left(N_{i}\right)=0, \mu_{i} /_{\left(\mathcal{L}_{i / N_{i}}\right)}$ sia completa e per ogni $s \in T_{i} \backslash N_{i}$ si abbia che $\varrho /_{E_{i(s)}}\left[\right.$ risp. $\left.s\left(\varrho /\left.\right|_{E_{\psi_{i}(s)}}\right)\right]$ è la topologia finale delle topologie $\varrho_{j} /_{\left(E_{i, j}\right)_{s}}\left[\right.$ risp. $\left.s\left(\varrho_{j} /_{\left(E_{i, j}\right)_{a}}\right)\right]$ rispetto alle $\varphi_{j_{\left(E_{i, j}\right)}, E_{w j(s)}}(j \in J)$

da (2.1.3) segue (2.1.2). 
Dimostrazrone. - Per il lemma 2.0 risulta che $\psi_{i}^{-1}\left(T \backslash T^{0}\right) \supset T_{i} \backslash \bigcap_{j \in J} T_{i, j}^{0}$ per ogni $i \in I$ e quindi, se vale (2.1.2), da (2.1.1) e dalla completezza delle misure $\mu_{i}(i \in I)$ segue che $T_{i} \backslash \bigcap_{j \in J} T_{i, j}^{0} \in \mathcal{L}_{i}$ e $\mu_{i}\left(T_{i} \backslash \bigcap_{j \in \mathcal{J}} T_{i, j}^{0}\right)=0$ per ogni $i \in I$ e, visto che $T_{i} \backslash T_{i, j}^{0} \subset$ $\subset T_{i} \backslash \bigcap_{k \in J} T_{i, k}^{0}(j \in J)$, utilizzando nuovamente la completezza delle misure $\mu_{i}(i \in I)$ si ottiene (2.1.3). Viceversa valga (2.1.3). Utilizzando (2.1.5), per il lemma 2.0 si ha che per ogni $i \in I$ esiste $M_{i} \subset N_{i}, M_{i} \in \mathfrak{L}_{i}$, con $\mu_{i}\left(\boldsymbol{M}_{i}\right)=0$ e tale che

(2.1.6) $\quad \psi_{i}^{-1}\left(T \backslash T^{0}\right)=\left(T_{i} \backslash \bigcap_{j \in J} T_{i, j}^{0}\right) \cup M_{i}$.

D'altra parte, se $J \subset N$, risulta che $T_{i} \backslash \bigcap_{j \in J} T_{i, j}^{0} \in \mathfrak{L}_{i}$ e $\mu_{i}\left(T_{i} \backslash \bigcap_{j \in \mathcal{J}} T_{i, j}^{0}\right)=0$ per ogni $i \in I$ e quindi, poichè vale (2.1.6) per ogni $i \in I$, usando (2.1.4) si ottiene (2.1.2).

2.2 Corollario. - Siano $T, T_{i}, \mathcal{L}, \mathcal{L}_{i}, \mu, \mu_{i},(X, \varrho),\left(X_{j}, \varrho_{j}\right), \psi_{i}, \varphi_{j}, E, E_{i, j}, f, f_{i, j}$, $T^{0}, T_{i, j}^{0}(i \in I, j \in J)$ come in $\left.A\right)$ del teorema 2.1. Allora, se $J=\{0\}$, se

(2.2.0) per ogni $i \in I$ ed $s \in T_{i}$ vale che $\left.\varrho\right|_{E_{\psi_{i}(s)}}\left[\right.$ risp. $\left.s\left(\left.\varrho\right|_{E_{p_{i}(s)}}\right)\right]$ è la topologia finale della topologia $\varrho_{0} /\left(E_{i_{0}, 0}\right)_{s}\left[\right.$ risp. $\left.s\left(\varrho_{0} /_{\left.\left(E_{i_{0}}\right)_{s}\right)}\right)\right]$ rispetto $a \varphi_{\left(E_{i, 0}\right)_{s}, E_{\psi_{i}(s)}}$

e se valgono (2.1.0) e (2.1.1), si ha che (2.1.2) implica (2.1.3).

Drmostrazione. - Basta sfruttare (2.2.0) e il lemma 2.0 per ottenere che $\psi_{i}^{-\mathrm{I}}(T \backslash$ $\left.\backslash T^{0}\right)=T_{i} \backslash T_{i, 0}^{0}(i \in I)$ e poi usare (2.1.1).

2.3 Thorema. - Siano $T, T_{i}, \mathfrak{L}, \mathfrak{L}_{i},(X, \varrho),\left(X_{i}, \varrho_{j}\right), \psi_{i}, \varphi_{j}, E, E_{i, j}, f, f_{i, j}(i \in I$, $j \in J)$ come in $A$ ) del teorema 2.1. Allora:

a) se valgono le seguenti condizioni

(2.3.0) $\psi_{i}^{-1}(A) \in \mathfrak{L}_{i}$ per ogni $i \in I$ e per ogni $A \in \mathbb{L}$ $\varphi_{j}^{-1}(B) \in \Re\left(\varrho_{j}\right)$ per ogni $j \in J$ e per ogni $B \in Q$,

da

(2.3.2) $E \in \mathfrak{L} \times \mathfrak{B}(\varrho), f$ è $(\mathcal{L} \times \mathcal{B}(\varrho)) / /_{E}$-misurabile

segue

$E_{i, j} \in \mathfrak{L}_{i} \times \mathcal{B}\left(\varrho_{j}\right), f_{i, j}$ è $\left(\mathfrak{L}_{i} \times \mathfrak{B}\left(\varrho_{j}\right)\right) /_{E_{i, j}}$-misurabile per ogni $i \in I, j \in J$ 
b) se vale la seguente condizione

$$
\left(\psi_{i} \times \varphi_{j}\right)^{-1}(A) \in \mathbb{L}_{i} \times \mathfrak{B}\left(\varrho_{j}\right) \text { per ogni } i \in I, j \in J \text { implica } A \in \mathfrak{L} \times \mathfrak{B}(\varrho)
$$

da (2.3.3) segue (2.3.2).

Dimostrazione. - Sia $a \in[-\infty, \infty]$. Allora, se vale (2.3.2), risulta che $E \in \mathfrak{L} \times$ $\times \mathscr{B}(\varrho)$ e $\left.\left.f^{-1}(] a, \infty\right]\right) \in \mathcal{L} \times \mathscr{B}(\varrho)$. Siano $i \in I, j \in J$; si ha che $E_{i, j}=\left(\psi_{i} \times \varphi_{j}\right)^{-1}(E) \in$ $\in \mathfrak{L}_{i} \times \mathfrak{B}_{(}\left(\varrho_{j}\right)$ e $\left.\left.\left.\left.\left(f_{i, j}\right)^{-1}(] a, \infty\right]\right)=\left(\psi_{i} \times \varphi_{j}\right)^{-1}\left(f^{-1}(] a, \infty\right]\right)\right) \in \mathfrak{L}_{i} \times \mathfrak{B}\left(\varrho_{j}\right)$ per $(2.3 .0),(2.3 .1) \mathrm{e}$ per a) e $h$ ) del lemma 1.7. Pertanto vale (2.3.3). Viceversa valga (2.3.3). Allora si ha che $E_{i, j} \in \mathfrak{L}_{i} \times \mathfrak{B}\left(\varrho_{j}\right)$ e $\left.\left.\left(f_{i, j}\right)^{-1}(] a, \infty\right]\right) \in \mathfrak{L}_{i} \times \mathfrak{B}\left(\varrho_{j}\right)$ per ogni $i \in I, j \in J$. Pertanto $\left(\psi_{i} \times \varphi_{j}\right)^{-1}(E) \in \mathfrak{L}_{i} \times \mathscr{B}\left(\varrho_{j}\right)$ e $\left.\left.\left(\psi_{i} \times \varphi_{j}\right)^{-1}\left(f^{-1}(] a, \infty\right]\right)\right) \in \mathfrak{L}_{i} \times \mathcal{B}\left(\varrho_{j}\right)$ per ogni $i \in I, j \in J$ e quindi usando (2.3.4) si ottiene che $E \in \mathcal{L} \times \Re(o)$ e $\left.\left.f^{-1}(] a, \infty\right]\right) \in \mathfrak{L} \times \mathscr{B}(Q)$, per cui vale (2.3.2).

2.4 Teorema. - Siano $(T, \tau),\left(T_{i}, \tau_{i}\right)$ spazi topologici, $\mathcal{L}, \mathfrak{L}_{i}, \mu, \mu_{i},(X, \varrho),\left(X_{j}, \varrho_{j}\right)$, $\psi_{i}, \varphi_{i}, E, E_{i, j}, f, f_{i, j}(i \in I, j \in J)$ come in $A$ ) del teorema 2.1 ; inoltre siano $\mathfrak{K} \subset \mathfrak{L}$, $\mathcal{H}_{i, j} \subset \mathcal{L}_{i}(i \in I, j \in J)$. Allora:

a) se valgono le seguenti condizioni

$\left(\psi_{i} \times \varphi_{j}\right)_{E_{i, j}, E}$ sono continue [risp. sequenzialmente continue] $(i \in I, j \in J)$

(2.4.1) $\psi_{i}^{-1}(K) \in \mathfrak{H}_{i, j}$ per ogni $i \in I, j \in J$ e per ogni $K \in \nVdash$

(2.4.2) per ogni $i \in I$ esiste $c_{i}>0$ tale che sia $\mu_{i}\left(\psi_{i}^{-1}(A)\right) \leqslant c_{i} \mu(A)$ per ogni $A \in \mathfrak{L}$ per cui $\psi_{i}^{-1}(A) \in \mathfrak{L}_{i}$,

da

(2.4.3) per ogni $n \in \boldsymbol{Z}_{+}$esiste $K_{n} \in \nVdash$ per cui sia $\mu\left(T \backslash K_{n}\right)<1 / n$ e $\left.f\right|_{E \cap\left(K_{n} \times X\right)}$ sia s.e.i. [risp. s.s.c.i.]

segue

(2.4.4) per ogni $i \in I, j \in J$ e $n \in \boldsymbol{Z}_{+}$esiste $K_{n, i, j} \in \mathscr{H}_{i, j}$ per cui sia $\mu_{i}\left(T_{i} \backslash K_{n, i, j}\right)<$ $<1 / n$ e $\left.f_{i, j}\right|_{E_{t, j} \cap\left(K_{n, i, j} \times X_{j}\right)}$ sia s.c.i. [risp. s.s.c.i.];

b) se $J \subset N$, se $F \in \mathcal{L}, F \supset \bigcup_{i \in I} \psi_{i}\left(T_{i}\right), E \subset F \times X$, se $\mu /_{(\mathcal{L} /(T \backslash F))}$ è $\{K \in \mathcal{K}: K \subset$ $\subset T \backslash F\}$-internamente regolare, se $K \cup H \in \mathcal{K}$ per ogni $K, H \in \mathcal{K}, K \subset F, H \subset T \backslash F^{\prime} \mathrm{e}$ se vale almeno uno dei due seguenti gruppi di condizioni:

i)

(2.4.5) per ogni $j \in J$, per ogni $\left(H_{n, i}: n \in Z_{+-1}, i \in I\right)$, con $H_{n, i} \in K_{i, j}$ tale che $\mu_{i}\left(T_{i} \backslash\right.$ $\left.\backslash H_{n, i}\right)<1 / n\left(n \in \boldsymbol{Z}_{+}, i \in I\right)$, esistono $\left(H_{n}: n \in \boldsymbol{Z}_{+}\right)$e $\left(k_{n, i}: n \in \boldsymbol{Z}_{+}, i \in I\right)$ con $H_{n} \in \mathcal{K}, H_{n} \subset F$ e $k_{n, i} \in Z_{+}$tali che $\psi_{i}^{-1}\left(H_{n}\right) \subset H_{k_{n, i}, i}$ per ogni $i \in I$ e $\mu(F \backslash$ $\left.\backslash H_{n}\right)<1 / n\left(n \in Z_{+}, i \in I\right)$, 
(2.4.6) esiste $\delta^{0}>0$ tale che, se $0<\delta<\delta^{0}$ e se $K \in \mathcal{L}$ è un'intersezione al più numerabile di elementi dell'unione delle famiglie $\left(H_{n}: n \in \boldsymbol{Z}_{+}\right)$corrispondenti alle famiglie $\left(H_{n, i}: n \in \boldsymbol{Z}_{+}, i \in I\right)$ secondo la $(2.4 .5)$ e per cui $\mu(F \backslash K)<\delta$, esista $K^{\delta} \in \pi, K^{\delta} \subset K$ per cui $\mu\left(\mathbb{R} \backslash K^{\delta}\right)<\delta$ e $\left.\quad(\tau \times \varrho)\right|_{E \cap\left(K^{\delta} \times X\right)} \quad$ [risp. $\left.s\left((\tau \times \varrho) /_{E \cap\left(K^{\delta} \times X\right)}\right)\right]$ sia la topologia finale delle topologie $\left.\left(\tau_{i} \times Q_{j}\right)\right|_{E_{i, j} \cap\left(\psi_{i}^{-1}\left(\mathbb{K}^{\delta}\right) \times X_{j}\right)}$ [risp. $\left.s\left(\left.\left(\tau_{i} \times \varrho_{j}\right)\right|_{E_{t, j} \cap\left(\psi_{i}^{-1}\left(K^{\delta}\right) \times X_{j}\right)}\right)\right]$ rispetto alle $\left(\psi_{i} \times \varphi_{j}\right)_{E_{i, j} \cap\left(\varphi_{i}^{-1}\left(K^{\delta}\right) \times X_{j}\right), E^{n} \cap\left(K^{\delta} \times \mathbb{X}\right)}$ $(i \in I, j \in J)$

ii) $X=\bigcup_{j \in J} \varphi_{j}\left(X_{j}\right)$,

(2.4.7) per ogni $j \in J$, per ogni $\left(H_{n, i}: n \in \boldsymbol{Z}_{+}, i \in I\right)$, con $H_{n, i} \in \mathfrak{K}_{i, j}$ tale che $\mu_{i}\left(T_{i} \backslash\right.$ $\left.\backslash H_{n, i}\right)<1 / n\left(n \in \boldsymbol{Z}_{+}, i \in I\right)$, esistono $\left(L_{n}: n \in \boldsymbol{Z}_{+}\right)$, con $L_{n} \subset I \quad\left(n \in \boldsymbol{Z}_{+}\right),\left(H_{n}\right.$ : $\left.n \in \boldsymbol{Z}_{+}\right)$e $\left(k_{n, i}: i \in L_{n}, n \in \boldsymbol{Z}_{+}\right)$con $H_{n} \in \mathfrak{K}$ e $k_{n, i} \in \boldsymbol{Z}_{+}\left(i \in L_{n}\right)$ tali che $\mu\left(F \backslash H_{n}\right)<1 / n, H_{n}=\bigcup_{i \in L_{n}} \psi_{i}\left(H_{k_{n, i}, i} \cap \psi_{i}^{-1}\left(H_{n}\right)\right)\left(n \in \boldsymbol{Z}_{+}\right)$e tali che valga almeno una delle quattro seguenti condizioni:

A) $\left(\psi_{i} \times \varphi_{j}\right)_{E_{t, j} \cap\left[\left(H_{k_{n, i}, i} \cap \psi_{i}^{-1}\left(H_{n}\right)\right) \times X_{j]}\right], E \cap\left(H_{n} \times X\right)}$ sia aperta [risp.

$\left(\psi_{i} \times \varphi_{j}\right)_{E_{1, j} \cap\left[\left(H_{k_{n, i}, i} \cap \psi_{i}^{-1}(K)\right) \times X_{j}\right], E \cap(K \times X)}$ sia sequenzialmente aperta per ogni $\left.K \in \mathcal{K}, K \subset \boldsymbol{H}_{n}\right]$ per ogni $i \in L_{n}, n \in \boldsymbol{Z}_{+}$,

B) $\left(\psi_{i} \times \varphi_{j}\right)_{E_{i, n} \cap\left[\left(H_{k_{n}, i,} \cap \psi_{i}^{-1}\left(H_{n}\right)\right) \times X_{j}\right], E \cap\left(\left(H_{n} \cap \psi_{i}\left(T_{i}\right)\right) \times X\right)}$ sia aperta [risp.

$\left(\psi_{i} \times \varphi_{j}\right)_{E_{i, j} \cap\left[\left(H_{k_{n, i}, i} \cap \psi_{i}^{-1}(K)\right) \times X_{j}\right], E \cap\left(\left(K \cap \psi_{i}\left(T_{i}\right)\right) \times X\right)}$ sia sequenzialmente aperta per

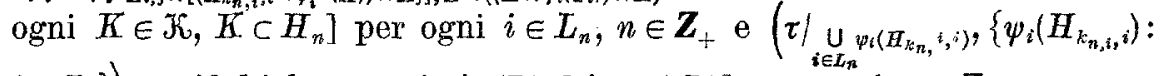
$\left.\left.i \in L_{n}\right\}\right)$ verifichi la proprietà (L) [risp. (sL)] per ogni $n \in \boldsymbol{Z}_{+}$,

C) $\left(\psi_{i} \times \varphi_{j}\right)_{E_{i, 3} \cap\left[\left(H_{k_{n, i}, i} \cap \varphi_{1}^{-1}\left(H_{n}\right)\right) \times X_{j}\right], E \cap\left(H_{n} \times \varphi_{j}\left(X_{j}\right)\right)}$ sia aperta [risp.

$\left(\psi_{i} \times \varphi_{j}\right)_{E_{i, j} \cap\left[\left(H_{k_{n}, i}, \cap \psi_{i}^{-1}(K)\right) \times X_{j}\right], E \cap\left(K \times \varphi_{j}\left(X_{j}\right)\right)}$ sia sequenzialmente aperta per ogni

$\left.K \in \mathcal{K}, K \subset H_{n}\right]$ per ogni $i \in L_{n}, n \in Z_{+}$e $\left(\varrho,\left\{\varphi_{j}\left(X_{j}\right): j \in J\right\}\right)$ verifichi la proprietà (L) [risp. (sL)],

D) $\left(\psi_{i} \times \varphi_{j}\right)_{E_{i, j} \cap\left[\left(H_{k_{n}, i}, \cap \psi_{i}^{-1}\left(H_{n}\right)\right) \times X_{j}\right], \mathbb{E} \cap\left[\left(H_{n} \cap \psi_{l}\left(T_{i}\right)\right) \times \varphi_{j}\left(X_{j}\right)\right]}$ sia aperta [risp.

$\left(\psi_{i} \times \phi_{j}\right)_{E_{i, j} \cap\left[\left(H_{k_{n}, i, i} \cap \varphi_{i}^{-1}(K)\right) \times X_{j}\right], E \cap\left[\left(K \cap \psi_{i}\left(T_{i}\right)\right) \times \varphi_{j}\left(X_{j}\right)\right]}$ sia sequenzialmente aperta per ogni $\left.K \in \mathcal{K}, K \subset H_{n}\right]$ per ogni $i \in L_{n}, n \in \boldsymbol{Z}_{+}$e $|\tau|_{i \in L_{n}} \psi_{i}\left(H_{k_{n}, i}, i\right)$, $\left.\left\{\psi_{i}\left(H_{k_{n, i}, i}\right): i \in L_{n}\right\}\right)$ e $\left(\varrho,\left\{\varphi_{j}\left(X_{j}\right): j \in J\right\}\right)$ verifichino la proprietà (L) [risp. (sL)] per ogni $n \in \boldsymbol{Z}_{+}$,

(2.4.8) esiste $\delta^{0}>0$ tale che, se $0<\delta<\delta^{0}$ e se $K \in \mathcal{L}$ è un'intersezione al più numerabile di elementi dell'unione delle famiglie $\left(H_{n}: n \in \boldsymbol{Z}_{+}\right)$corrispondenti alle famiglie $\left(H_{n, i}: n \in \boldsymbol{Z}_{+}, i \in I\right)$ secondo la (2.4.7) e per cui $\mu(\mathbb{F} \backslash K)<\delta$, esista $K^{\delta} \in \mathcal{K}, K^{\delta} \subset K$ per eui $\mu\left(F \backslash K^{\delta}\right)<\delta$

da $(2.4 .4)$ segue $(2.4 .3)$.

Druostrazione. - Valga (2.4.3) e siano $i \in I, j \in J$. Sia $n \in \boldsymbol{Z}_{+}$e sia $m_{n, i} \in \boldsymbol{Z}_{+}$ tale che $c_{i} / m_{n, i} \leqslant 1 / n$ (ove $c_{i}$ è come in (2.4.2)); allora basta scegliere $K_{n, i, j}=\psi_{i}^{-1}\left(K_{m_{n, i}}\right)$ 
e si ottiene che $K_{n, i, j} \in \mathscr{H}_{i, j}$ per (2.4.1), $\mu_{i}\left(T_{i} \backslash K_{n, i, j}\right)=\mu_{i}\left(\psi_{i}^{-1}\left(T \backslash K_{m_{n, i}}\right)\right) \leqslant c_{i} \mu(T \backslash$ $\left.\backslash K_{m_{n, i}}\right)<c_{i} / m_{n, i} \leqslant 1 / n$ per $(2.4 .2)$ e inoltre

$\left.f_{i, j}\right|_{E_{i, j} \cap\left(K_{n, i, j} \times X_{j}\right)}=\left.\left(\left.f \circ\left(\psi_{i} \times \varphi_{j}\right)\right|_{E_{i, j}}\right)\right|_{E_{i, j} \cap\left(K_{n, i, j} \times X_{j}\right)}=\left(\left.f\right|_{E \cap\left(K_{m_{n, i}} \times \mathbb{X}\right)}\right) \circ\left(\left.\left(\psi_{i} \times \varphi_{j}\right)\right|_{E_{i, j} \cap\left(\mathbb{K}_{n, i, j} \times X_{j}\right)}\right)$

è s.c.i. [risp. s.s.c.i.] per (2.4.0) [risp. tenendo conto del fatto che, utilizzando $f$ ) del teorema 1.1, si prova facilmente che da (2.4.0) segue che $\left(\psi_{i} \times \varphi_{j}\right)_{E_{i, j} \cap\left(K_{n, i, j} \times X_{j}\right), E \cap\left(K_{m_{n, i}} \times X\right)}$ sono sequenzialmente continue $(i \in I, j \in J)]$. Viceversa valga (2.4.4). Si proverà dapprima che

(2.4.9) per ogni $n \in \boldsymbol{Z}_{+}$esiste $K_{n}^{\prime} \in \nVdash, K_{n}^{\prime} \subset F$ per cui sia $\mu\left(F \backslash K_{n}^{\prime}\right)<1 / n$ e $\left.f\right|_{E \cap\left(K_{n}^{i} \times X\right)}$ sia s.c.i. [risp. s.s.c.i.].

Valga dapprima i) e sia $j \in J$. Siano $H_{n}^{(j)} \in \mathcal{K}, H_{n}^{(j)} \subset F$. relativi ai $K_{n, i, j}(i \in I)$ come in (2.4.5) e siano $k_{n, i, j} \in Z_{+}(i \in I)$ tali che $\psi_{i}^{-1}\left(H_{w_{0}}^{(j)}\right) \subset K_{k_{n, i, j}, i, j}$ per ogni $i \in I$ $\left(n \in \boldsymbol{Z}_{+}\right)$; sia inoltre $K_{n}^{\prime}$ relativo come in (2.4.6) all'intersezione $\bigcap_{j \in J} H_{n 2^{j+1}}^{(j)}$ e a $\delta=1 / n$ $\left(\mu\left(F \backslash \bigcap_{j \in J} H_{n 2^{j+1}}^{(j)}\right)<\sum_{j \in J} 1 /\left(n 2^{j+1}\right) \leqslant 1 / n\right)$ per ogni $n \in Z_{+}$per cui $1 / n<\delta^{j}$ (cioè per ogni $\left.n \geqslant\left[1 / \delta^{0}\right]+1\right)$ e sia $K_{n}^{\prime}=K_{\left[1 / \delta^{0}\right]+1}^{\prime}$ per ogni $n \in \boldsymbol{Z}_{+}, n<\left[1 / \delta^{0}\right]+1$. Allora $\boldsymbol{K}_{n}^{\prime} \in \mathcal{K}$, $\mu\left(\boldsymbol{F}^{\backslash} \backslash \boldsymbol{K}_{n}^{\prime}\right)<1 / n\left(n \in \boldsymbol{Z}_{+}\right)$. Sia ora $a \in[-\infty, \infty]$; allora per (2.4.6) si ha che $\left.\left.\left(\left.f\right|_{E \cap\left(\mathbb{K}_{n}^{\prime} \times X\right)}\right)^{-1}(] a, \infty\right]\right)$ è in $\left.(\tau \times \varrho)\right|_{E \cap\left(K_{n}^{\prime} \times X\right)}\left[\right.$ risp. in $\left.s\left(\left.(\tau \times \varrho)\right|_{E \cap\left(K_{n}^{\prime} \times X\right)}\right)\right]$ se per ogni $i \in I, j \in J$ l'insieme $\left.\left.\left.\left.\left(\psi_{i} \times \varphi_{j}\right)^{-1}\left[\left(\left.f\right|_{E \cap\left(K_{n}^{\prime} \times X\right)}\right)^{-1}(] a, \infty\right]\right)\right]=\left(f_{i, j} / I_{i, j} \cap\left(\psi_{i}^{-1}\left(K_{n}^{\prime}\right) \times X_{j}\right)\right)^{-1}(] a, \infty\right]\right)$ è in $\left.\left(\tau_{i} \times \varrho_{j}\right)\right|_{\mathbb{E}_{i, j} \cap\left(\psi_{i}^{-1}\left(K_{n}^{i}\right) \times X_{j}\right)}\left[\right.$ risp. in $\left.s\left(\left(\tau_{i} \times Q_{j}\right) /_{E_{i, j} \cap\left(\psi_{i}^{-1}\left(\pi_{n}^{\prime}\right) \times X_{j}\right)}\right)\right]\left(n \in Z_{+}\right)$, il che è vero per (2.4.4) e poichè $\psi_{i}^{-1}\left(K_{n}^{\prime}\right) \subset \psi_{i}^{-1}\left(\bigcap_{l \in J} H_{n 2^{l+1}}^{(l)}\right) \subset \psi_{i}^{-1}\left(H_{n 2^{j+1}}^{(j)} \subset K_{k_{2 n^{j+1}, i, j, i, j}}\right.$ per ogni $i \in I$, $j \in J$ e $n \in \boldsymbol{Z}_{+}, n \geqslant\left[1 / \delta^{0}\right]+1, \psi_{i}^{-1}\left(K_{n}^{\prime}\right)=\psi_{i}^{-1}\left(K_{\left[1 / \delta^{0}\right]+1}^{\prime}\right)$ per ogni $n \in \boldsymbol{Z}_{+}, n<\left[1 / \delta^{0}\right]+1$. Pertanto vale (2.4.9). Valga ora ii) e sia $j \in J$. Siano $L_{n, i} \subset I, H_{n}^{(j)} \in \mathcal{K}$ e $k_{n, i, i} \in Z_{+}$ relativi ai $K_{n, i_{s} j}(i \in I)$ come in (2.4.7) $\left(n \in \boldsymbol{Z}_{+}\right)$; sia inoltre $K_{n}^{\prime}$ relativo come in (2.4.8) all'intersezione $\bigcap_{j \in J} H_{n 2^{j+1}}^{(j)}$ e a $\delta=1 / n\left(\mu\left(E^{\nearrow} \bigcap_{j \in J} H_{n 2^{2+1}}^{(j)}\right)<\sum_{j \in J} 1 /\left(n 2^{j+1}\right) \leqslant 1 / n\right)$ per ogni $n \in \boldsymbol{Z}_{+}$per cui $1 / n<\delta^{0}$ (cioè per ogni $n \geqslant\left[1 / \delta^{0}\right]+1$ ) e sia $K_{n}^{\prime}=K_{\left[1 / \delta^{0}\right]+1}^{\prime}$ per ogni $n \in \boldsymbol{Z}_{+}, n<\left[1 / \delta^{0}\right]+1$. Allora $K_{n}^{\prime} \in \mathfrak{K}, \mu\left(F \backslash K_{n}^{\prime}\right)<1 / n\left(n \in \boldsymbol{Z}_{+}\right)$. Sia ora $n \in \boldsymbol{Z}_{+} ;$per ogni $j \in J$ risulta che $K_{n}^{f} \subset H_{n 2^{j+1}}^{(j)}=\bigcup_{i \in L_{n} 2^{i+1}, j} \psi_{i}\left(K_{k_{n 2^{2+1}, i, j, i, j}} \cap \psi_{i}^{-1}\left(H_{n 2^{j+1}}^{(j)}\right)\right.$, da cui

$$
K_{n}^{\prime}=\bigcup_{i \in L_{n 2^{j+1}, j}} \psi_{i}\left(K_{k_{n 2^{j+1}, i, j, i, j}} \cap \psi_{i}^{-1}\left(K_{n}^{\prime}\right)\right)
$$

e quindi se $a \in[-\infty, \infty]$ risulta che

$$
\begin{aligned}
& \left.\left.\left(\left.f\right|_{E \cap\left(R_{n}^{2} \times X\right)}\right)^{-1}(] a, \infty\right]\right)=
\end{aligned}
$$

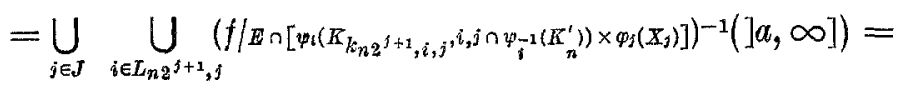

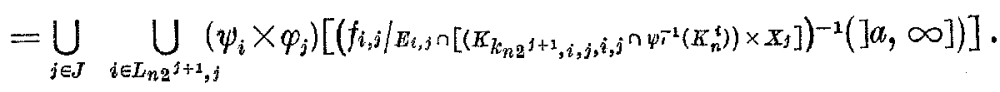


Allora se vale $A)$ risulta che l'insieme in $(2.4 .11)$ è in $(\tau \times \varrho) /_{E \cap\left(\mathbb{K}_{n}^{\prime} \times X\right)}$ [risp. in $\left.s\left(\left.(\tau \times Q)\right|_{E \cap\left(K_{n}^{\prime} \times X\right)}\right)\right]$, dove basta tener conto del teorema 1.25 con l'ipotesi $\left.b\right)$ [risp. basta tener conto del fatto che $\left.K_{n}^{\prime} \in \Pi\right]$ e pertanto vale (2.4.9). Negli altri casi si ha che esistono $B_{n, i, j}=E \cap\left[\psi_{i}\left(K_{k_{n 2^{j+1}, i, j}, i, j} \cap \psi_{i}^{-1}\left(K_{n}^{\prime}\right)\right) \times \varphi_{j}\left(X_{j}\right)\right] \subset E \cap\left(K_{n}^{f} \times X\right)$ tali che

(2.4.12) $\left.\left.\left(f /_{B_{n, i, j}}\right)^{-1}(] a, \infty\right]\right)$ sia aperto [risp. sequenzialmente aperto] nel seguente spazio: $E \cap\left(\left(K_{n}^{\prime} \cap \psi_{i}\left(T_{i}\right)\right) \times X\right)$ (se vale $\left.\left.B\right)\right), E \cap\left(K_{n}^{\prime} \times \varphi_{j}\left(X_{j}\right)\right)$ (se vale $\left.C\right)$ ), $E \cap\left[\left(K_{n}^{\prime} \cap \psi_{i}\left(T_{i}\right)\right) \times \varphi_{j}\left(X_{j}\right)\right]$ (se vale $\left.\left.D\right)\right)\left(i \in L_{n 2^{j+1}, j}, j \in J\right)$

(si è tenuto conto del teorema 1.25 con l'ipotesi $b$ ) [risp. del fatto che $\left.K_{n}^{\prime} \in \nVdash\right]$ ]). Se ora per assurdo l'insieme in (2.4.11) non è in $\left.(\tau \times \varrho)\right|_{\mathbb{E} \cap\left(K_{n}^{2} \times X\right)}$ [risp. in $s((\tau \times$ $\left.\left.\times \varrho) /_{E \cap\left(K_{n}^{\prime} \times X\right)}\right)\right]$, esistono

$$
\left.\left.\left.\left.x_{k} \in E \cap\left(K_{n}^{\prime} \times X\right), x_{k} \notin\left(\left.f\right|_{E \cap\left(K_{n}^{\prime} \times X\right)}\right)^{-1}(] a, \infty\right]\right), x \in\left(f /_{E \cap\left(K_{n}^{\prime} \times X\right)}\right)^{-1}(] a, \infty\right]\right)
$$

$(k \in K$, ove $(K, \succ)$ è un insieme diretto) [risp. $(k \in N)]$ tali che $x_{k} \rightarrow x$ in $\tau \times \varrho$. Ora per (2.4.10) si ha che

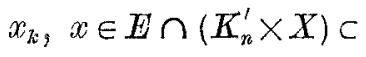

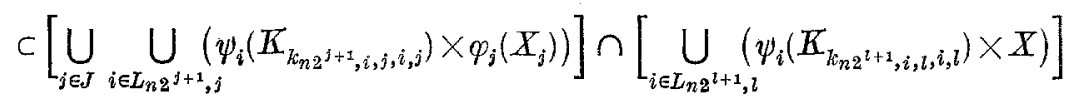

per ogni $l \in J, k \in K$ [risp. $l \in J, k \in N]$ e quindi per le ipotesi fatte riguardo alla verifica della proprietà $(\mathrm{L})$ [risp. (sL)] e per il teorema 1.18 esistono $(H, \cdot \succ)$ insieme diretto, $(\beta(h))_{h \in H}$ estratta da $(k)_{k \in K}\left[\right.$ risp. $\left(k_{h}\right)_{h \in N}$ successione strettamente crescente di naturali] ed esistono rispettivamente: $i_{l} \in I_{n 2^{i+1, l}}$ (ove $l$ è un elemento di $J$ tale che $x \in T \times \varphi_{l}\left(X_{l}\right)$ ) (se vale $\left.B\right)$ ), $j \in J$ (se vale $\left.C\right)$ ), $j \in J$ ed $i \in L_{n 2^{1+1, j}}$ (se vale $D$ )) per cui $x_{\beta(h)}$ [risp. $x_{k_{h}}$ ], $x$ siano rispettivamente in: $\psi_{i_{l}}\left(K_{k_{n 2} 2^{l+1}, i_{l}, i_{l}, l}\right) \times X$ (se vale $\left.B\right)$ ), $T \times \varphi_{j}\left(X_{j}\right)$ (se vale $\left.C\right)$ ), $\psi_{i}\left(K_{k_{n 2^{j+1}, i, j, i, j}}\right) \times \varphi_{j}\left(X_{j}\right)$ (se vale $\left.\left.D\right)\right)(h \in H)$ [risp. $\left.(h \in N)\right]$. Pertanto per (2.4.12) esiste $h^{0} \in \mathbb{H}\left[\right.$ risp. $\left.h^{0} \in N\right]$ tale che se $h \cdot>h^{0}\left[\right.$ [risp. $h>h^{0}$ ] si abbia $x_{\beta(h)}$ [risp. $x_{k_{h}}$ ] appartenente rispettivamente a: $\left.\left.\left(f /_{B_{n, i_{t}, t}}\right)^{-1}(] a, \infty\right]\right)(\mathrm{se}$ vale $\left.\left.B)), \bigcup_{i \in L_{n 2^{j+1, j}}}\left(f /_{B_{n, i, j}}\right)^{-1}(] a, \infty\right]\right)$ (se vale 0$\left.\left.\left.)\right), \quad\left(\left.f\right|_{B_{n, i, j}}\right)^{-1}(] a, \infty\right]\right)$ (se vale $\left.D\right)$ ) e quindi per (2.4.11) e tenendo conto di come sono stati definiti gli insiemi $B_{n, i, j}$ $\left(i \in L_{n 2^{3+1}, j}, j \in J\right)$ si ha che in ciascuno dei tre casi $\left.\left.\left.B\right), C\right), D\right)$, se $h \cdot>h^{0}$ [risp. $\left.h>h^{0}\right]$, risulta che $x_{\beta(h)}$ [risp. $\left.x_{k_{h}}\right]$ è in $\left.\left.\left(f /_{E \cap\left(K_{n}^{\prime} \times X\right)}\right)^{-1}(] a, \infty\right]\right)$, il che è assurdo. Pertanto vale (2.4.9).

Sia $n \in \boldsymbol{Z}_{+}$. Basta ora considerare $K_{n}^{\prime \prime} \in \mathcal{K}, K_{n}^{\prime \prime} \subset T \backslash F$ tale che sia $\mu\left[(T \backslash F) \backslash K_{n}^{\prime \prime}\right]<$ $<1 /(2 n)$ (l'esistenza di un tale insieme $K_{n}^{\prime \prime}$ è assicurata dalla $\{K \in \mathfrak{K}: K \subset T \backslash F\}$ -

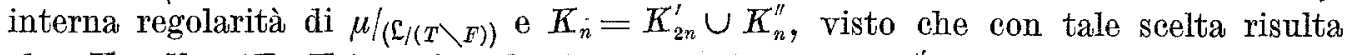
che $K_{n} \in \pi, \mu\left(T \backslash K_{n}\right)<1 / n$ ed $E \cap\left(K_{n} \times X\right)=E \cap\left(K_{2 n}^{\prime} \times X\right)$, per cui $\left.f\right|_{E \cap\left(K_{n} \times X\right)}$ è s.c.i. [risp. s.s.c.i.]. 
2.5 Osservazione. - Si noti che, anche grazie ad alcuni risultati del $\S 1$, si possono dare condizioni sufficienti affinchè siano verificate le ipotesi dei teoremi 2.1, 2.3 e 2.4 .

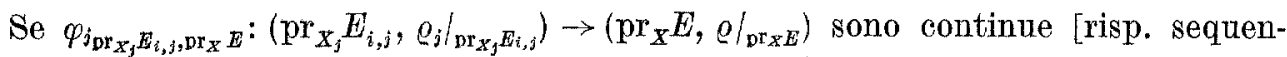
zialmente continue] $(i \in I, j \in J)$ o anche se $\varphi_{j}:\left(X_{j}, \varrho_{j}\right) \rightarrow(X, \varrho)$ sono continue [risp. sequenzialmente continue] $(j \in J)$ l'ipotesi (2.1.0) è verificata [risp. l'ipotesi (2.1.0) è verificata poichè basta usare $f$ ) del teorema 1.1]; per ottenere una parte di (2.1.4) si può utilizzare il teorema 1.27 a); nei teoremi 1.14 e 1.23 sono date condizioni per ottenere (2.1.5) e, se $J=\{0\}$ e $\varphi_{0}$ è surgettiva, l'ipotesi riguardante la verifica della proprietà (L) [risp. (sL)] fatta in $b^{\prime}$ ) del teorema 1.23 è sempre vera; se $\varphi_{j}$ sono continue allora (2.3.1) è ovviamente vera; $b$ ) del teorema 1.27 garantisce

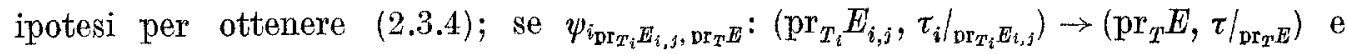
$\varphi_{\mathrm{prr}_{X}, E_{i, j}, \mathrm{pr}_{X} E}:\left(\mathrm{pr}_{X_{j}} E_{i, j}, \varrho_{j} /_{\mathrm{pr}_{X j} E_{t, j}}\right) \rightarrow\left(\mathrm{pr}_{X} E, \varrho / /_{\mathrm{pr}_{X} E}\right)$ sono continue [risp. sequenzialmente continue] $(i \in I, j \in J)$ allora vale (2.4.0) ovviamente [risp. vale (2.4.0) utilizzando $f$ ) del teorema 1.1]; a) del lemma 1.11 fornisce ipotesi che implicano (2.4.1) nel caso in cui $\mathcal{K}_{i, j} \supset\left\{K: K\right.$ compatto in $\left.\tau_{i}\right\}(i \in I, j \in J), \pi \subset\{K: K$ compatto in $\tau$ \} e se inoltre $\psi_{i}$ è continua per ogni $i \in I$ allora si ha (2.4.1) anche nel caso in cui $\mathfrak{H}_{i, j} \supset\left\{K: K\right.$ compatto e chiuso in $\left.\tau_{i}\right\}(i \in I, j \in J), \pi \subset\{K: K$ compatto e chiuso in $\tau\} ; a), b$ ) e $c$ ) del teorema 1.29 relativi a $F$ suggeriscono condizioni per verificare rispettivamente (2.4.5), la prima parte di (2.4.7) e (2.4.7) con la condizione $A$ ) (e, se $F=\bigcup_{i \in I} \psi_{i}\left(T_{i}\right)$, in $(1.29 .3)$ e in (1.29.4) relativi a $F$ è automaticamente soddisfatta l'ipotesi che sia $\left.\mu\left[F \backslash\left(\bigcup_{i \in I} \psi_{i}\left(T_{i}\right)\right)\right]=0\right)$ (e, se $\psi_{i}$ è continua, si ha che $\psi_{i}(K)$ è compatto in $\tau$ per ogni $K$ compatto in $\tau_{i}$, per cui vale la prima parte di (1.29.2) nel caso in eui $\mathfrak{H}_{i} \subset\left\{K: K\right.$ compatto in $\left.\tau_{i}\right\}(i \in I), \pi \supset\{K: K$ compatto in $\left.\tau\}\right)$; si noti anche che, se valgono (1.29.2) e (1.29.4) relativamente a $F$, allora la prima parte di (2.4.7) è verificata con degli insiemi $L_{n}$ finiti $\left(n \in \boldsymbol{Z}_{+}\right)$; la $\{K \in \nVdash: K \subset F\}$-interna regolarità di $\mu_{\left(\mathbb{L}_{/ F}\right)}$ implica sia $(2.4 .8)$ e sia la prima parte di (2.4.6) (e, se $\mathcal{K}=\{K: K$ compatto e chiuso in $\tau\}$ (o anche se $\mathcal{K}=\{K: K$ chiuso in $\tau\}$ ), allora (2.4.8) e la prima parte di (2.4.6) sono ovviamente verificate considerando $K^{8}=$ $=K(\delta>0))$; per la verifica della seconda parte di (2.4.6) sono date ipotesi nei teoremi 1.21 e 1.23 ; inoltre sono date condizioni per la verifica della seconda parte di (2.4.7) nel lemma 1.4 [risp. nel teorema 1.25].

\section{BIBLIOGRAFIA}

[1] E. Acerbi - N. Fusco, Semicontinuity Problems in the Calculus of Variations, di prossima pubblicazione su "Arch. Rational Mech. Anal.".

[2] L. D. Berkovitz, Lower Semicontinuity of Integral Functionals, Trans. Amer. Math. Soc., 192 (1974), pp. 51-57. 
[3] A. Bottaro ARUfFo, Su alcune estensioni del teorema di Scorza Dragoni, di prossima pubblicazione.

[4] N. Bourbaki, General Topology, Hermann, Addison-Wesley, 1966.

[5] F. E. Browder, Remarls on the Direct Method of the Calculus of Variations, Arch Rational Mech. Anal,, 20 (1965), pp. 251-258.

[6] O. Caligaris - P. Oliva, Un teorema di esistenza per problemi di Lagrange in spazi di Banach, Atti Accad. Naz. Lincei Rend. Cl. Sci. Fis. Mat, Natur., (8) 61 (1976), pp. $571-579$.

[7] C. Castarng - M. Valadier, Conven Analysis and Measurable Multifunetions, Lecture Notes n. 580, Springer, 1977.

[8] L. CeSARI, Lower Semicontinuity and Lower Closure Theorems Without Seminormality Conditions, Ann. Mat. Pura Appl., (4) 98 (1974), pp. 381-397.

[9] M. DolCher, Topologie e strutture di convergenza, Ann. Scuola Norm. Sup. Pisa Cl. Sci., (3) 14 (1960), pp. 63-92.

[10] I. EkELAND - R. TEmam, Analyse convexe et problèmes variationnelles, Dunod, Gauthier Villars, 1974.

[11] P. R. Halmos, Measure Theory, D. Van Nostrand Company, 1950.

[12] P. R. Halmos, A Hilbert Space Problem Book, Springer, 1974.

[13] A. D. IoFFE, On Lower Semicontinuity of Integral Funetionals. I, SIAM J. Control Optim., 15 (1977), pp. 521-538.

[14] J. L. KELLEx, General Topology, Springer, 1975.

[15] P. Marceldini - C. Sbordone, Semicontimuity Problems in the Oaleulus of Variations, Nonlinear Anal., 4 (1980), pp. 241-257.

[16] C. OLECH, A Characterization of $L_{1}$-Weals Lower Semicontinuity of Integral Functionals, Bull. Acad. Polon. Sci. Sér. Sci. Math., 25 (1977), pp. 135-142.

[17] J. von NeUMany, Zur Algebra der Funltionaloperationen und Theorie der normalen Operatoren, Math. Ann., 102 (1930), pp. 370-427.

[18] S. BARoN - S. Leader, Sequential Topologies, Amer. Math. Monthly, 73 (1966), pp. 677-678 (problema \# 5299).

[19] J. R. Boone, Sequentially Quotient Mappings, Czechoslovak Math. J., 26 (1976), pp. 174-182.

[20] S. P. FrankLIN, Spaces in which sequences suffice, Fund. Math., 57 (1965), pp. 107-115. 